\title{
WEIGHTED HARDY SPACES: SHIFT INVARIANT AND COINVARIANT SUBSPACES, LINEAR SYSTEMS AND OPERATOR MODEL THEORY
}

\author{
JOSEPH A. BALL AND VLADIMIR BOLOTNIKOV \\ In memory of Bela Sz.-Nagy, a fine mathematician and leading operator theorist
}

\begin{abstract}
The Sz.-Nagy-Foias model theory for C.0 contraction operators combined with the Beurling-Lax theorem establishes a correspondence between any two of four kinds of objects: shift-invariant subspaces, operator-valued inner functions, conservative discrete-time input/state/output linear systems, and C.0 Hilbert-space contraction operators. We discuss an analogue of all these ideas in the context of weighted Hardy spaces over the unit disk and an associated class of hypercontraction operators.
\end{abstract}

\section{INTRODUCTION}

A couple of seminal developments in nonselfadjoint operator theory in the middle part of the last century was the Sz.-Nagy dilation theorem and the Sz.-Nagy-Foias model theory (we refer to the second edition of the Sz.Nagy-Foias monograph [68] (with additional authors H. Bercovici and L. Kerchy) for a comprehensive treatment which includes a thorough discussion of later related developments). The Sz.-Nagy dilation theorem asserts that any Hilbert-space contraction operator can be lifted to a coisometry (as well as dilated to a unitary operator), while the Sz.-Nagy-Foias model theory associates with any completely nonunitary contraction operator $T$ a characteristic function $\Theta_{T}$ which is a contractive analytic operator-valued function between two coefficient Hilbert spaces $\mathcal{U}$ and $\mathcal{Y}$. The characteristic function $\Theta=\Theta_{T}$ has the additional property that it is pure in the sense that there are no nonzero subspaces $\mathcal{U}_{0}$ and $\mathcal{Y}_{0}$ of $\mathcal{U}$ and $\mathcal{Y}$ respectively such that $\left.\Theta(z)\right|_{\mathcal{U}_{0}}$ reduces to a constant unitary operator from $\mathcal{U}_{0}$ onto $\mathcal{Y}_{0}$. Conversely, starting with any pure contractive analytic function $(\Theta(z), \mathcal{U}, \mathcal{Y})$, there is a functional model Hilbert space $\mathcal{H}(\Theta)$ and a completely nonunitary canonical-model contraction operator $T=T(\Theta)$ acting on $\mathcal{H}(\Theta)$ so

1991 Mathematics Subject Classification. 47A57.

Key words and phrases. Operator-valued functions, weighted Hardy space, Bergman inner functions, Beurling-Lax theorem, hypercontraction operators, dilation theory, characteristic function.

The second author's research was supported by the Plumeri Award of the College of William and Mary. 
that $T$ and $T(\Theta)$ are unitarily equivalent in case $\Theta=\Theta_{T}$. An important motivating special case is the $C_{.0}$ case where the characteristic function is closely entangled with the Beurling-Lax-Halmos (or simply Beurling-Lax for short) theorem associating an inner function with a shift-invariant subspace of $H^{2}$ (operator-valued inner function for the case of a higher-multiplicity shift acting on $H^{2}(\mathcal{Y}):=H^{2} \otimes \mathcal{Y}$ for a coefficient Hilbert space $\mathcal{Y}$ ); see [28, 56, 48]. Indeed, the Sz.-Nagy-Foias model theory for the $C_{.0}$ case can be summed up as follows: roughly, apart from manageable degeneracies, there is a one-to-one correspondence between four kinds of objects: (1) shiftinvariant subspaces $\mathcal{M} \subset H^{2}(\mathcal{Y})$, (2) operator-valued inner functions $\Theta$, (3) unitary colligation matrices $\left[\begin{array}{ll}A & B \\ C & D\end{array}\right]:\left[\begin{array}{l}\mathcal{X} \\ \mathcal{U}\end{array}\right] \rightarrow\left[\begin{array}{l}\mathcal{X} \\ \mathcal{Y}\end{array}\right]$, and (4) C.0-contraction operators $T$. Note that the Beurling-Lax theorem gives the correspondence between a shift-invariant subspace $\mathcal{M} \subset H^{2}(\mathcal{Y})$ and an inner function $\Theta$ via $\mathcal{M}=\Theta \cdot H^{2}(\mathcal{Y})$. Given an inner function $\Theta$, the Sz.-Nagy-Foias theory tells us that $\Theta$ coincides with the characteristic function $\Theta_{T}$ for the $C_{.0}$ contraction operator $T:=\left.P_{\mathcal{M}^{\perp}} M_{z}\right|_{\mathcal{M}^{\perp}}$. The formula for a characteristic function then leads to a realization for $\Theta=\Theta_{T}$ of the form

$$
\Theta(z)=D+z C(I-z A)^{-1} B
$$

with $U=\left[\begin{array}{ll}A & B \\ C & D\end{array}\right]$ unitary. Conversely, if $U=\left[\begin{array}{cc}A & B \\ C & D\end{array}\right]$ is unitary with $A$ of class $C_{0}$., one can verify that $\Theta(z)$ given by (1.1) is inner. Associated with a shift-invariant subspace $\mathcal{M} \subset H^{2}(\mathcal{Y})$ is the contraction operator

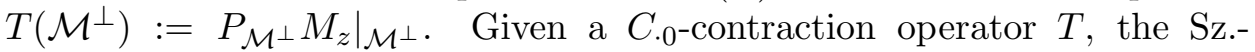
Nagy-Foias characteristic function $\Theta_{T}$ provides an inner function so that we recover $T$ up to unitary equivalence in the form $T=\left.P_{\mathcal{M}} M_{z}\right|_{\mathcal{M}}$ with $\mathcal{M}=\Theta_{T} \cdot \mathcal{U}$. In short, starting with an object of any one of the four types listed above, there is a recipe for passing to its equivalent representative in any one of the three remaining types.

It should be mentioned that related notions of characteristic function and associated operator model theory appeared in the work of Livšic and the Ukrainian operator-theory school (see [34, 58, 59]) as well as in the work of de Branges and Rovnyak (see [32, 33]). The characteristic function also appears in other guises, namely, as the scattering function in the setting of Lax-Phillips scatterings (see [57, 3, 55, 69, 70]) where there is also a close connection with the geometry of the Sz.-Nagy-Foias dilation space, as well as the transfer function of a conservative discrete-time linear system (or the scattering function for a lossless LCR circuit) (see [54]).

While the Sz.-Nagy-Foias model theory in its original form had a tremendous influence and applications for the theory of a single contraction operator on a Hilbert space, we focus here on extensions to more general settings. Also we focus only on the aspects of dilation theory, characteristic function and associated operator model theory; this leaves out another key and influential component of the theory, namely the Commutant Lifting Theorem which has also seen lots of extensions to more general settings (see e.g. [45, 64]). There were first obtained compelling extensions of the 
Sz.-Nagy dilation theory to classes other than contractions, including multivariable versions involving commutative operator-tuples rather than a single operator, in the work of Agler [4, 5], Müller [66], Müller-Vasilescu [67, Athavale [15], Curto-Vasilescu [38, 39], Pott [79, Ambrozie-Engliš-Müller [9], and Arazy-Engliš [10]. An early identification of a general operatoralgebra setting for dilation theory which indeed influenced some of the work mentioned above was achieved by Arveson [12, 13.

The general settings for which there has also been identified a characteristic function to this point are more limited compared to those where a dilation theory exists. There was early work of Abrahamse-Douglas [1, 2] and Ball [16] and continuing with [60, 23, 24] where function theory on the disk is replaced by function theory on a finitely-connected planar domain (or more generally bordered Riemann surface of dividing type); this work is also closely tied up with the appropriate notion of a Beurling-Lax theorem for this setting. A more complete analogue of the whole Sz.-Nagy-Foias model theory is the extension to the setting where the single operator $T$ is replaced by a freely noncommutative row contraction. Here we say that the $d$-tuple $T=\left(T_{1}, \ldots, T_{d}\right)$ of operators on the Hilbert space $\mathcal{H}$ is a row contraction if the row matrix $\left[\begin{array}{lll}T_{1} & \cdots & T_{d}\end{array}\right]$ is contractive as an operator from the direct sum space $\mathcal{H}^{d}=\bigoplus_{j=1}^{d} \mathcal{H}$ into $\mathcal{H}$; we refer to the work of Bunce, Frazho, and Popescu [35, 46, 75, 77, for the dilation theory aspects and Popescu [76] for the characteristic function aspects; the work of Ball-BolotnikovFang [20] drew out the system-theory aspects while that of Ball-Vinnikov [26] extended these results from the the completely non-coisometric setting to the general completely nonunitary setting. There has also been work (see e.g. the work of Muhly-Solel [65]) extending the Sz.-Nagy-Foias model theory to more abstract operator-algebra settings. There is a parallel dilation theory, characteristic function, model theory, and Beurling-Lax theorem for the case of a commutative row contraction (see the work of Drury [42, Arveson [14, Bhattacharyya-Eschmeier-Sarkar [30, 31], Ball-Bolotnikov [19], McCullough-Trent [62]), as well as more flexible settings simultaneously containing the freely noncommutative case and the commutative case [29, 78]. There is also an operator model theory and a version of the characteristic function for the setting where the single contraction operator is replaced by a family of contraction operators $T_{n} \in \mathcal{L}\left(\mathcal{H}_{n+1}, \mathcal{H}_{n}\right)(n=\cdots-1,0,1, \ldots)$ and the characteristic function is the input/output map of a conservative time-varying linear system (see the papers of Constantinescu [36, 37] and Alpay-Ball-Peretz [8]). We also mention that the Hilbert-module setting for the Sz.-Nagy-Foias model theory was pursued in [41, 64, 40].

Generally speaking, a distinguishing feature of the cases where the characteristic function appears versus the cases where there is only a dilation theory and associated model theory without a characteristic-function invariant is that the associated positive kernel is not of the type now called a Pick kernel (see e.g. [6] for the terminology). A first step away from this 
restriction was in the work of Olofsson [72, 74] who introduced a characteristic function for the class of $n$-hypercontractions, i.e., operators $T$ for which $\sum_{k=0}^{m}(-1)^{k}\left(\begin{array}{c}m \\ k\end{array}\right) T^{* k} T^{k} \geq 0$ for $1 \leq m \leq n$. The class of $n$-hypercontractions is closely tied to the function theory for the weighted Bergman space $\mathcal{A}_{n}(\mathcal{Y})$ over the unit disk (where $\mathcal{Y}$ is a coefficient Hilbert space) having operatorvalued reproducing kernel equal to $k_{n}(z, \zeta) \cdot I_{\mathcal{Y}}:=\frac{1}{(1-z \zeta)^{n}} I_{\mathcal{Y}}$. A Beurling-Lax theorem for the Bergman space setting has been of interest to the functiontheoretic operator-theory community since the 1970s but has turned out to be much more difficult to come by; the results obtained are necessarily of a more delicate form, with the most progress just since the 1990s. There eventually evolved a notion of Bergman inner function to be a function $\Theta$ which maps a coefficient Hilbert space isometrically onto a wandering subspace $\mathcal{E} \subset \mathcal{A}_{n}(\mathcal{Y})$ for the Bergman shift operator $S_{n}$ equal to multiplication by the coordinate function $M_{z}: f(z) \mapsto z f(z)$ on $\mathcal{A}_{n}(\mathcal{Y})$. Here $\mathcal{E}$ is a wandering subspace means only that $\mathcal{E}$ is orthogonal to $S_{n}^{k} \mathcal{E}$ for all $k>0$; a key distinction from the Hardy space case is that it does not follow that $S_{n}^{k} \mathcal{E}$ is orthogonal to $S_{n}^{k^{\prime}} \mathcal{E}$ for distinct positive integers $k, k^{\prime}$. Then at least we get a Beurling-like representation $\mathcal{M}$ as the closure of $\Theta \mathcal{U}[z]$ (where $\mathcal{U}[z]$ is the linear space of polynomials with coefficients in $\mathcal{U}$ and $\Theta$ is a Bergman inner function with $\Theta \cdot \mathcal{U}$ equal to the wandering subspace $\left.\mathcal{E}=\mathcal{M} \ominus S_{n} \mathcal{M}\right)$. Bergman inner functions were first constructed by Hedenmalm as the solution of an extremal problem (see [50, 51]). The biharmonic Green function was introduced shortly thereafter to prove the contractive divisor property in a conceptually better way extending the result to an $L^{p}$-setting by Duren, Khavinson, Shapiro and Sundberg (see e.g. 43]). When the shift-invariant subspace $\mathcal{M} \subset \mathcal{A}_{n}(\mathcal{Y})$ is pure in the sense that $\mathcal{E}:=\mathcal{M} \ominus S_{n} \mathcal{M}$ is generating for $\mathcal{M}$ (meaning that $\mathcal{M}=\overline{\operatorname{span}}_{k \geq 0} S_{n}^{k} \mathcal{E}$ ), one gets at least a Beurling-type representation of the form $\mathcal{M}=\overline{\operatorname{span}} S_{n}^{k} \mathcal{E}$; the fact that this holds in general for the unweighted case $n=2$ was first proved by Aleman-Richter-Sundberg [7. Shimorin [81, 82] noticed that many of these ideas can be developed in a purely operator-theoretic setting where $S_{n}$ is replaced by a left-invertible Hilbert-space operator $T$. Additional analysis of the wandering-subspace property has been developed in [52, 61, 71, 83]. There are now available two monographs [44,53] leading the reader through many of these developments.

The work of Olofsson offered a new direction for the computation and construction of Bergman inner functions by introducing ideas from linear system theory whereby Bergman inner functions have a transfer-functionlike realization

$$
\Theta(z)=D+z C\left(\sum_{k=1}^{n}(I-z A)^{-k}\right) B
$$

for a certain colligation matrix $U=\left[\begin{array}{cc}A & B \\ C & D\end{array}\right]$ constructed explicitly from the invariant subspace $\mathcal{M}$. Alternatively, one could start with the $n$-hypercontraction $A=T^{*}$ (assuming that $A$ is a $C_{0}$. $n$-hypercontraction), use the dilation 
theory of Agler [5] to model $A$ as the restriction of $S_{n}^{*}$ to an $S_{n}^{*}$-invariant subspace $\mathcal{M}^{\perp} \subset \mathcal{A}_{n}(\mathcal{Y})$, and identify explicitly the Bergman-inner function $\Theta$ associated with the $S_{n}$-invariant subspace $\mathcal{M}$ as the characteristic function of $T$. The explicit formula of the type (1.2) for $\Theta_{T}$ is very much like the Sz.-Nagy-Foias formula for the characteristic function for a contraction operator $T$, but now one must work with certain $n$-level generalized defect operators $D_{n, T}$ and $D_{n, T^{*}}$ rather than the standard Sz.-Nagy-Foias defect operators $D_{T}=\left(I-T^{*} T\right)^{1 / 2}$ and $D_{T^{*}}=\left(I-T T^{*}\right)^{1 / 2}$ in the Sz.-Nagy-Foias theory.

Our own paper [18] followed up on this work of Olofsson by drawing out further the system-theory aspects and introducing several alternate Beurling-Lax-type representations for an $S_{n}$-invariant subspace $\mathcal{M}$ of $\mathcal{A}_{n}(\mathcal{Y})$. The present paper extends the work of [18] in two respects: (1) we replace the Bergman space $\mathcal{A}_{n}(\mathcal{Y})$ with a more general weighted Hardy space $H_{\boldsymbol{\beta}}^{2}(\mathcal{Y})$ described below, and (2) in addition to Beurling-Lax representation theorems, we here explicitly define a characteristic function (more precisely, characteristic function family) for a $\boldsymbol{\beta}$ - $C_{.0} *$ - $\boldsymbol{\beta}$-hypercontraction operator $T$ on a Hilbert space $\mathcal{X}$ and obtain a complete Sz.-Nagy-Foias dilation and model theory for this class of operators.

In detail, the class of weighted Hardy spaces which we consider is as follows. Given a sequence $\boldsymbol{\beta}=\left\{\beta_{j}\right\}_{j \geq 0}$ of positive numbers, the weighted Hardy space $H_{\boldsymbol{\beta}}^{2}$ is defined as the set of all functions analytic on the open unit disk $\mathbb{D}$ and with finite norm $\|f\|_{H_{\beta}^{2}}$ given by

$$
\|f\|_{H_{\boldsymbol{\beta}}^{2}}^{2}=\sum_{j=0}^{\infty} \beta_{j}\left|f_{j}\right|^{2} \quad \text { if } \quad f(z)=\sum_{j=0}^{\infty} f_{j} z^{j} .
$$

Polynomials are dense in $H_{\boldsymbol{\beta}}^{2}$ and the monomials $\left\{z^{k}\right\}_{k \geq 0}$ form an orthogonal set uniquely defining the weight sequence $\boldsymbol{\beta}$ by $\beta_{j}=\left\|z^{j}\right\|^{2}$ for $j \geq 0$. A general reference for such spaces and the associated weighted shift operators is the article of Shields [80].

For a Hilbert space $\mathcal{Y}$, we denote by $H_{\boldsymbol{\beta}}^{2}(\mathcal{Y})$ the tensor product Hilbert space $H_{\boldsymbol{\beta}}^{2} \otimes \mathcal{Y}$ which can be identified as

$$
H_{\boldsymbol{\beta}}^{2}(\mathcal{Y})=\left\{f(z)=\sum_{k \geq 0} f_{k} z^{k}:\|f\|_{H_{\boldsymbol{\beta}}^{2}(\mathcal{Y})}^{2}:=\sum_{k \geq 0} \beta_{k} \cdot\left\|f_{k}\right\|_{\mathcal{Y}}^{2}<\infty\right\} .
$$

If $\lim \inf \beta_{j}^{\frac{1}{j}} \geq 1$, then the power series

$$
R_{\boldsymbol{\beta}}(z)=\sum_{j=0}^{\infty} \beta_{j}^{-1} z^{j}
$$


converges on the open unit disk $\mathbb{D}$. The function

$$
K_{\boldsymbol{\beta}}(z, \zeta)=R_{\boldsymbol{\beta}}(z \bar{\zeta})=\sum_{j=0}^{\infty} \beta_{j}^{-1} \cdot z^{j} \bar{\zeta}^{j}
$$

turns out to be the reproducing kernel for $H_{\boldsymbol{\beta}}^{2}$ in the sense that $z \mapsto K_{\boldsymbol{\beta}}(z, \zeta)$ belongs to $H_{\boldsymbol{\beta}}^{2}$ and the equality $\left\langle f, K_{\boldsymbol{\beta}}(\cdot, \zeta)\right\rangle_{H_{\boldsymbol{\beta}}^{2}}=f(\zeta)$ holds for every $\zeta \in \mathbb{D}$ and $f \in H_{\boldsymbol{\beta}}^{2}$.

We will write 1 for the sequence $\boldsymbol{\beta}$ with $\beta_{j}=1$ for all $j \geq 0$. The space $H_{1}^{2}(\mathcal{Y})$ is the classical vector Hardy space $H^{2}(\mathcal{Y})$ of the unit disk. Another important example is given by the weight sequence $\boldsymbol{\beta}_{\alpha}=\left\{\beta_{\alpha, k}\right\}_{k \geq 0}$ with

$$
\beta_{\alpha, k}=\frac{k !}{\alpha(\alpha+1) \cdots(\alpha+k-1)}=\frac{k ! \Gamma(\alpha)}{\Gamma(\alpha+k)}
$$

for any fixed $\alpha>1$. The space $H_{\boldsymbol{\beta}_{\alpha}}^{2}$ equals the Bergman space $A_{\alpha-2}^{2}$ of $\mathcal{Y}$-valued functions $f$ analytic on $\mathbb{D}$ and such that

$$
\|f\|_{A_{\alpha-2}^{2}}^{2}=(\alpha-1) \int_{\mathbb{D}}\|f(z)\|_{\mathcal{Y}}^{2}\left(1-|z|^{2}\right)^{\alpha-2} d A(z)<\infty
$$

where $d A$ is the planar Lebesgue measure normalized so that $A(\mathbb{D})=1$.

The shift operator $S_{\boldsymbol{\beta}}$ on $H_{\boldsymbol{\beta}}^{2}$ is defined by $S_{\boldsymbol{\beta}}: f(z) \mapsto z f(z)$ and simple inner-product calculations show that its adjoint $S_{\boldsymbol{\beta}}^{*}$ is given by

$$
S_{\boldsymbol{\beta}}^{*} f=\sum_{k=0}^{\infty} \frac{\beta_{k+1}}{\beta_{k}} \cdot f_{k+1} z^{k} \quad \text { if } \quad f(z)=\sum_{k=0}^{\infty} f_{k} z^{k} .
$$

In this paper we will be primarily interested in subspaces of $H_{\boldsymbol{\beta}}^{2}$ which are invariant either under $S_{\boldsymbol{\beta}}$ or under $S_{\boldsymbol{\beta}}^{*}$. For the rest of the paper, we assume that the weight sequence $\boldsymbol{\beta}=\left\{\beta_{j}\right\}_{j \geq 0}$ meets the following conditions. Firstly we assume that

$$
\liminf \beta_{j}^{\frac{1}{j}}=1, \quad \beta_{0}=1 \quad \text { and } \quad 1 \leq \frac{\beta_{j}}{\beta_{j+1}} \leq M \quad \text { for all } j \in \mathbb{Z}_{+}
$$

and some $M \geq 1$. The two first conditions are normalizing and thus, nonrestrictive. It is seen from (1.6) that $\left\|S_{\boldsymbol{\beta}}^{*}\right\|=\sup _{j \geq 0} \frac{\beta_{j+1}}{\beta_{j}}$ and thus, the third condition in (1.7) means that the shift operator $S_{\boldsymbol{\beta}}: H_{\boldsymbol{\beta}}^{2} \rightarrow H_{\boldsymbol{\beta}}^{2}$ is contractive and left-invertible. Secondly, we assume that the reciprocal power series

$$
\sum_{j=0}^{\infty} c_{j} z^{j}:=\frac{1}{R_{\boldsymbol{\beta}}(z)}=\left(\sum_{j=0}^{\infty} \beta_{j}^{-1} z^{j}\right)^{-1}
$$


belongs to the Wiener class $W^{+}$, that is, the coefficients $\left\{c_{j}\right\}_{j \geq 0}$ appearing in (1.8) are absolutely summable:

$$
\text { if } c_{0}=1 \text { and recursively } c_{n}=-\sum_{j=0}^{n-1} c_{j} \beta_{n-j}^{-1} \text {, then } \sum_{j=0}^{\infty}\left|c_{j}\right|<\infty \text {. }
$$

We remark that the sequence $\boldsymbol{\beta}_{\alpha}$ defined in (1.5) meets all the above assumptions. In particular, when $\alpha=n$ is a positive integer, then $\frac{1}{R_{\boldsymbol{\beta}}(z)}$ is even a polynomial. A more general example is given by the function

$$
R_{\boldsymbol{\beta}}(z)=\sum_{j=0}^{\infty} \beta_{j}^{-1} z^{j}=\frac{1}{(1-z)^{\alpha}(1-z g(z))}
$$

where $\alpha>1$ and $g$ is a function in $W^{+}$with non-negative Taylor coefficients at the origin having no zeros in the closed unit disk. An open question is whether this Wiener-algebra assumption (1.9) can be weakened in such a way that the results of this paper continue to hold.

The operator-model theory developed in this paper is as follows. We establish a correspondence between three types of objects: (1) $S_{\boldsymbol{\beta}}$-invariant subspaces $\mathcal{M} \subset H_{\boldsymbol{\beta}}(\mathcal{Y}),(2) \boldsymbol{\beta}$-inner function families $\left\{\Theta_{k}\right\}_{k \geq 0},(3)$ a family of colligation matrices $U_{k}$ of the type considered in Section 2 below which satisfy additional metric constraints discussed in Section 5 below ( $\boldsymbol{\beta}$-unitary colligation family), and (4) $\boldsymbol{\beta}$ - $C_{.0} *$ - $\boldsymbol{\beta}$-hypercontraction operators $T$. Here the term $\boldsymbol{\beta}-C_{0} 0$ applied to a Hilbert space operator $T$ is a strengthening of the standard notion of $C .0$ as used in the book [68] (i.e., the property that $T^{* n} \rightarrow 0$ strongly as $n \rightarrow \infty$ ) tailored to the sequence $\boldsymbol{\beta}$ (see Definition 7.1 below). Associated with any colligation family is a certain function family $\left\{\Theta_{k}\right\}_{k \geq 0}$ which has the interpretation as the transfer function for a certain time-varying input/state/output linear system described in Section 2; when the colligation family is also $\boldsymbol{\beta}$-unitary, the associated family of functions is an inner function family and it is natural to say that the associated time-varying linear system is $\boldsymbol{\beta}$-unitary: this is worked out in Sections 5 and 6.2. This gives the correspondence between $\boldsymbol{\beta}$-inner function families and $\boldsymbol{\beta}$-unitary colligation families or equivalently $\boldsymbol{\beta}$-unitary linear systems. The Beurling-Lax piece of the correspondence referred above is the representation of an $S_{\boldsymbol{\beta}}$-invariant subspace $\mathcal{M}$ in terms of a $\boldsymbol{\beta}$-inner function family $\left\{\Theta_{k}\right\}_{k \geq 0}$ as $\mathcal{M}=\bigoplus_{k=0}^{\infty} S_{\boldsymbol{\beta}}^{k} \Theta_{k} \cdot \mathcal{U}_{k}$ : this is worked out in Section 6.2 below. Given an $S_{\boldsymbol{\beta}}$-invariant subspace $\mathcal{M} \subset H_{\boldsymbol{\beta}}(\mathcal{Y})$, we show in Section 4 that $\left.P_{\mathcal{M}} S_{\boldsymbol{\beta}}\right|_{\mathcal{M}}$ is a $\boldsymbol{\beta}$ - $C_{.0} *$ - $\boldsymbol{\beta}$-hypercontraction. Given any $\boldsymbol{\beta}$-C.0 *- $\boldsymbol{\beta}$-hypercontraction $T$, in Section 7 we associate its $\boldsymbol{\beta}$-inner characteristic function family $\left\{\Theta_{T, k}\right\}_{k \geq 0}$ so that we recover $T$ up to unitary equivalence as $T=\left.P_{\mathcal{M}} S_{\boldsymbol{\beta}}\right|_{\mathcal{M}}$ with $\mathcal{M}=\bigoplus_{k=0}^{\infty} S_{\boldsymbol{\beta}}^{k} \Theta_{T, k} \mathcal{U}_{k}$. In Section 7 we also show how to go directly from a given $\boldsymbol{\beta}$-inner function family $\left\{\Theta_{k}\right\}_{k \geq 0}$ to a $\boldsymbol{\beta}$-unitary colligation family which realizes $\left\{\Theta_{k}\right\}_{k \geq 0}$, i.e., we obtain explicit formulas 
for the colligation operator-matrices $U_{k}=\left[\begin{array}{cc}A & B_{k} \\ C & D_{k}\end{array}\right]$ constructed from the function family $\left\{\Theta_{k}\right\}_{k \geq 0}$.

A natural follow-up project to this paper is to develop this model theory for $*$ - $\boldsymbol{\beta}$-hypercontractions with the $\boldsymbol{\beta}-C .0$ hypothesis removed. For a given *- $\boldsymbol{\beta}$-hypercontraction $T$, it does hold that $T$ being $\boldsymbol{\beta}$ - $C .0$ implies that $T$ is in fact $C_{.0}$ but we have not been able to resolve the converse except for the special case where $\beta_{k}=\frac{k !(n-1) !}{(k+n-1) !}$ (i.e., $\boldsymbol{\beta}$ of the form $\boldsymbol{\beta}_{\alpha}$ as in (1.5) with $\alpha=n$ a positive integer as studied in $[72,174,18]$ ) (see Remark 4.4 below); another open question for future work is to resolve this issue.

After the present Introduction, in Section 2 we give a time-domain systemtheoretic interpretation for the class of inner function families coming up in Section 6.2. Section 3 presents the $\boldsymbol{\beta}$-analogues of standard notions from systems theory concerning observability operators, observability gramians, and associated Stein equations and inequalities which will be needed in the sequel. Section 4 applies these constructions to the model setting where the system operator is the restriction of the backward $\boldsymbol{\beta}$-shift $S_{\boldsymbol{\beta}}^{*}$ to an invariant subspace $\mathcal{M}^{\perp} \subset H_{\boldsymbol{\beta}}^{2}(\mathcal{Y})$. Section 5 separates out the consequences of the metric properties associated with a $\boldsymbol{\beta}$-unitary colligation family; these are used for the construction of the $\boldsymbol{\beta}$-inner function family Beurling-Lax representer for an $S_{\boldsymbol{\beta}}$-invariant subspace $\mathcal{M} \subset H_{\boldsymbol{\beta}}^{2}(\mathcal{Y})$ in Section 6.2. Section 6.1 and 6.3 discuss other types of Beurling theorems in parallel to those

developed in our earlier work [18] for the special case $\beta_{j}=\frac{j !(n-1) !}{(j+n-1) !}$ for a positive integer $n$; in particular, the Beurling representation theorem in Section 6.3 corresponds to that of Olofsson in [74]. The final Section 7 defines the characteristic function family and develops the operator-model theory for the class of $\boldsymbol{\beta}-C_{.0}, *-\boldsymbol{\beta}$-hypercontraction operators. There also is given the functional-model form for the colligation matrix associated with a given $\boldsymbol{\beta}$-inner function family $\left\{\Theta_{k}\right\}_{k \geq 0}$.

Finally it is a pleasure to thank the anonymous referee for a thorough reading and review of the manuscript which led to a number of improvements in the final version.

\section{System-THEORETiC Motivation}

For $\mathcal{U}$ and $\mathcal{Y}$ any pair of Hilbert spaces, we use the notation $\mathcal{L}(\mathcal{U}, \mathcal{Y})$ to denote the space of bounded, linear operators from $\mathcal{U}$ to $\mathcal{Y}$. For $\mathcal{X}$ a single Hilbert space, we shorten the notation $\mathcal{L}(\mathcal{X}, \mathcal{X})$ to $\mathcal{L}(\mathcal{X})$. Let $\boldsymbol{\beta}$ be a given weight sequence, let $\mathcal{X}, \mathcal{Y}$ and $\mathcal{U}_{k}(k \geq 0)$ be Hilbert spaces, let

$$
A \in \mathcal{L}(\mathcal{X}), \quad C \in \mathcal{L}(\mathcal{X}, \mathcal{Y}), \quad B_{k} \in \mathcal{L}\left(\mathcal{U}_{k}, \mathcal{X}\right), \quad D_{k} \in \mathcal{L}\left(\mathcal{U}_{k}, \mathcal{Y}\right)
$$


be bounded linear operators, and let us consider the associated discrete-time time-variant linear system

$$
\Sigma_{\boldsymbol{\beta}}:\left\{\begin{aligned}
x(j+1) & =\frac{\beta_{j}}{\beta_{j+1}} \cdot A x(j)+\frac{1}{\beta_{j+1}} \cdot B_{j} u(j), \\
y(j) & =C x(j)+\frac{1}{\beta_{j}} \cdot D_{j} u(j)
\end{aligned}\right.
$$

with $x(j)$ taking values in the state space $\mathcal{X}, u(j)$ taking values in the inputspace $\mathcal{U}_{j}$ and $y(j)$ taking values in the output-space $\mathcal{Y}$. If we let the system evolve on the nonnegative integers $j \in \mathbb{Z}_{+}$, then the whole trajectory $\{u(j), x(j), y(j)\}_{j \in \mathbb{Z}_{+}}$is determined from the input signal $\{u(j)\}_{j \geq 0}$ and the initial state $x(0)$ according to the formulas

$$
\begin{aligned}
& x(j)=\beta_{j}^{-1} \cdot\left(A^{j} x(0)+\sum_{\ell=0}^{j-1} A^{j-\ell-1} B_{\ell} u(\ell)\right), \\
& y(j)=\beta_{j}^{-1} \cdot\left(C A^{j} x(0)+\sum_{\ell=0}^{j-1} C A^{j-\ell-1} B_{\ell} u(\ell)+D_{j} u(j)\right) .
\end{aligned}
$$

Formula (2.2) is established by simple induction arguments, while (2.3) is obtained by straightforward substituting of (2.2) into the second equation in (2.1). The integral form of the system equations (2.1) is the map from initial-state/input signal to state trajectory/output signal

$$
T\left(\Sigma_{\boldsymbol{\beta}}\right)=\left[\begin{array}{ll}
T\left(\Sigma_{\boldsymbol{\beta}}\right)_{11} & T\left(\Sigma_{\boldsymbol{\beta}}\right)_{12} \\
T\left(\Sigma_{\boldsymbol{\beta}}\right)_{21} & T\left(\Sigma_{\boldsymbol{\beta}}\right)_{22}
\end{array}\right]:\left[\begin{array}{c}
x(0) \\
\{u(j)\}_{j \geq 0}
\end{array}\right] \mapsto\left[\begin{array}{l}
\{x(j)\}_{j>0} \\
\{y(j)\}_{j \geq 0}
\end{array}\right]
$$

determined by solving the system equations (2.1) recursively. Our main interest here will be only in the two pieces

$$
\mathcal{O}_{\boldsymbol{\beta}}:=T\left(\Sigma_{\boldsymbol{\beta}}\right)_{21}: x(0) \mapsto\{y(j)\}_{j \geq 0},
$$

the map from initial state to output signal induced by setting the input signal equal to 0 and usually called the observation map, and the map

$$
\mathbf{T}_{\boldsymbol{\beta}}:=T\left(\Sigma_{\boldsymbol{\beta}}\right)_{22}:\{u(j)\}_{j \geq 0} \mapsto\{y(j)\}_{j \geq 0}
$$

from input signal to output signal determined by setting the initial state equal to 0 , usually called the input-output map for the system $\Sigma_{\boldsymbol{\beta}}$. From (2.3) we see that $\mathcal{O}_{\boldsymbol{\beta}}$ and $T_{\boldsymbol{\beta}}$ have the explicit matrix representations

$$
\mathcal{O}_{\boldsymbol{\beta}}=\left[\beta_{i}^{-1} C A^{i}\right]_{i \geq 0}, \quad\left[\mathbf{T}_{\boldsymbol{\beta}}\right]_{i, j}= \begin{cases}0 & \text { for } i<j, \\ \beta_{i}^{-1} D_{i} & \text { for } i=j, \\ \beta_{i}^{-1} C A^{i-1-j} B_{j} & \text { for } i>j, \quad 0 \leq i, j .\end{cases}
$$

Let us introduce the $Z$-transformed input, state and output signals

$$
\widehat{u}(z)=\sum_{k=0}^{\infty} u(k) z^{k}, \quad \widehat{x}(z)=\sum_{k=0}^{\infty} x(k) z^{k}, \quad \widehat{y}(z)=\sum_{k=0}^{\infty} y(k) z^{k} .
$$


(Note that $\widehat{u}(z)$ is merely formal since $u(j) \in \mathcal{U}_{j}$ and in general the input spaces are distinct linear spaces for distinct indices $j$ and hence there is no ambient linear space in which to take the sum.) To write the $Z$-transformed version of the system-trajectory formulas (2.2)-(2.3), we introduce the standard backward shift operator $S_{1}^{*}$ acting on formal power series according to

$$
S_{1}^{*}: \sum_{n=0}^{\infty} a_{n} z^{n} \mapsto \sum_{n=0}^{\infty} a_{n+1} z^{n} .
$$

We next introduce backward shifts of the function (1.4) by letting

$$
R_{\boldsymbol{\beta}, k}(z):=\left(S_{1}^{* k} R_{\boldsymbol{\beta}}\right)(z)=\sum_{j=0}^{\infty} \beta_{k+j}^{-1} z^{j}
$$

so that $R_{\boldsymbol{\beta}, 0}(z)=R_{\boldsymbol{\beta}}(z)$. For every Hilbert space operator $A \in \mathcal{L}(\mathcal{X})$ having spectral radius at most one, we can define operator-valued functions

$$
R_{\boldsymbol{\beta}, k}(z A)=\sum_{j=0}^{\infty} \beta_{k+j}^{-1} A^{j} z^{j} \quad \text { for all } \quad k \geq 0
$$

defined for $z \in \mathbb{D}$. Multiplying both sides of (2.2) by $z^{j}$ and summing up over $j \geq 0$, we get, on account of (2.7),

$$
\begin{aligned}
\widehat{x}(z) & =\left(\sum_{j=0}^{\infty} \beta_{j}^{-1} A^{j} z^{j}\right) x(0)+\sum_{k=1}^{\infty}\left(\sum_{j=k}^{\infty} \beta_{j}^{-1} A^{j-k} z^{j}\right) B_{k-1} u(k-1) \\
& =R_{\boldsymbol{\beta}}(z A) x(0)+\sum_{k=1}^{\infty} z^{k}\left(\sum_{j=0}^{\infty} \beta_{j+k}^{-1} A^{j} z^{j}\right) B_{k-1} u(k-1) \\
& =R_{\boldsymbol{\beta}}(z A) x(0)+\sum_{k=1}^{\infty} z^{k} R_{\boldsymbol{\beta}, k}(z A) B_{k-1} u(k-1) \\
& =R_{\boldsymbol{\beta}}(z A) x(0)+\sum_{k=0}^{\infty} z^{k+1} R_{\boldsymbol{\beta}, k+1}(z A) B_{k} u(k) .
\end{aligned}
$$

The same procedure applied to (2.3) gives

$$
\begin{aligned}
\widehat{y}(z) & =C R_{\boldsymbol{\beta}}(z A) x(0)+\sum_{k=0}^{\infty} z^{k}\left(\beta_{k}^{-1} D_{k}+z C R_{\boldsymbol{\beta}, k+1}(z A) B_{k}\right) u(k) \\
& =\mathcal{O}_{\boldsymbol{\beta}, C, A} x(0)+\sum_{k=0}^{\infty} z^{k} \Theta_{k}(z) u(k),
\end{aligned}
$$

where

$$
\mathcal{O}_{\boldsymbol{\beta}, C, A}: x \mapsto C R_{\boldsymbol{\beta}}(z A) x=\sum_{j=0}^{\infty}\left(\beta_{j}^{-1} C A^{j} x\right) z^{j}
$$


is the $\boldsymbol{\beta}$-observability operator (the $Z$-transform of the time-domain observation operator $\mathcal{O}_{\beta}(2.4)$ ) and where

$$
\Theta_{k}(z)=\beta_{k}^{-1} D_{k}+z C R_{\boldsymbol{\beta}, k+1}(z A) B_{k} \quad(k=0,1, \ldots)
$$

is a family of transfer functions encoding the $Z$-transform of the time-domain input-output operator $\mathbf{T}_{\boldsymbol{\beta}}(2.5)$ :

$$
\mathbf{T}_{\boldsymbol{\beta}}:\{u(j)\}_{j \geq 0} \mapsto\{y(j)\}_{j \geq 0} \Leftrightarrow \sum_{j=0}^{\infty} y(j) z^{j}=\sum_{j=0}^{\infty} \Theta_{j}(z) u(j) z^{j} .
$$

Note that we recover the classical time-invariant case by setting $\beta_{j}=1$ for all $j$ and by taking $B_{j}=B$ and $D_{j}=D$ independent of $j$; in this case $\Theta_{j}(z)=D+z C(I-z A)^{-1} B$ is independent of $j$ and the formula on the right in (2.11) reduces to

$$
\widehat{y}(z)=\Theta_{0}(z) \cdot \widehat{u}(z) .
$$

These observations suggest that the following terminology will be useful.

Definition 2.1. When the function family $\left\{\Theta_{k}\right\}_{k \geq 0}$ is connected with the colligation family $\left\{U_{k}=\left[\begin{array}{ll}A & B_{k} \\ C & D_{k}\end{array}\right]\right\}_{k \geq 0}$ as in (2.10), we say that the colligation family $\left\{U_{k}\right\}_{k \geq 0}$ provides a $\boldsymbol{\beta}$-realization for the function family $\left\{\Theta_{k}\right\}_{k \geq 0}$, and that the function family $\left\{\Theta_{k}(z)\right\}_{j \geq 0}$ is the $\boldsymbol{\beta}$-transfer function family for the colligation family $\left\{U_{k}\right\}_{k \geq 0}$ and the associated system $\Sigma_{\boldsymbol{\beta}}$ (2.1).

\section{Observability operators and gramians, Stein Equalities and INEQUALITIES}

Formula (2.9) associates with any output pair $(C, A)$ (i.e., $C \in \mathcal{L}(\mathcal{X}, \mathcal{Y})$ and $A \in \mathcal{L}(\mathcal{X}))$ the $\boldsymbol{\beta}$-observability operator $\mathcal{O}_{\boldsymbol{\beta}, C, A}$. In case $\mathcal{O}_{\boldsymbol{\beta}, C, A}$ is bounded as an operator from $\mathcal{X}$ into $H_{\boldsymbol{\beta}}^{2}(\mathcal{Y})$, we say that the pair $(C, A)$ is $\boldsymbol{\beta}$-output-stable. If $(C, A)$ is $\boldsymbol{\beta}$-output stable, then the $\boldsymbol{\beta}$-observability gramian

$$
\mathcal{G}_{\boldsymbol{\beta}, C, A}:=\left(\mathcal{O}_{\boldsymbol{\beta}, C, A}\right)^{*} \mathcal{O}_{\boldsymbol{\beta}, C, A}
$$

is bounded on $\mathcal{X}$ and can be represented via the series

$$
\mathcal{G}_{\boldsymbol{\beta}, C, A}=\sum_{k=0}^{\infty} \beta_{k}^{-1} \cdot A^{* k} C^{*} C A^{k}
$$

converging in the strong operator topology; see e.g. [49, Problem 120]. We will also make use of the shifted versions of $\mathcal{O}_{\boldsymbol{\beta}, C, A}$ and $\mathcal{G}_{\boldsymbol{\beta}, C, A}$ given by

$$
\mathfrak{O}_{\boldsymbol{\beta}, C, A}^{(k)} x=\sum_{j=0}^{\infty} \beta_{j+k}^{-1}\left(C A^{j} x\right) z^{j} \quad \text { and } \quad \mathfrak{G}_{\boldsymbol{\beta}, C, A}^{(k)}=\sum_{j=0}^{\infty} \beta_{j+k}^{-1} A^{* j} C^{*} C A^{j}
$$

for $k \geq 0$. It follows from (3.2) and formula (1.3) for the norm in $H_{\boldsymbol{\beta}}^{2}(\mathcal{Y})$ that

$$
\mathfrak{G}_{\boldsymbol{\beta}, C, A}^{(k)}=\left(S_{\boldsymbol{\beta}}^{k} \mathfrak{O}_{\boldsymbol{\beta}, C, A}^{(k)}\right)^{*} S_{\boldsymbol{\beta}}^{k} \mathfrak{O}_{\boldsymbol{\beta}, C, A}^{(k)} .
$$


On the other hand, comparing formulas (3.2) for $k=0$ with (2.9) and (3.1) gives

$$
\mathfrak{O}_{\boldsymbol{\beta}, C, A}^{(0)}=\mathcal{O}_{\boldsymbol{\beta}, C, A} \quad \text { and } \quad \mathfrak{G}_{\boldsymbol{\beta}, C, A}^{(0)}=\mathcal{G}_{\boldsymbol{\beta}, C, A} .
$$

Proposition 3.1. If $\beta_{j} / \beta_{j+1} \leq M$ for all $j \geq 0$ and $(C, A)$ is a $\boldsymbol{\beta}$-outputstable pair, then

$$
\left\|\mathfrak{O}_{\boldsymbol{\beta}, C, A}^{(k)}\right\| \leq M^{k} \cdot\left\|\mathcal{O}_{\boldsymbol{\beta}, C, A}\right\|, \quad \mathfrak{G}_{\boldsymbol{\beta}, C, A}^{(k)} \leq M^{k} \cdot \mathcal{G}_{\boldsymbol{\beta}, C, A} .
$$

Furthermore, the weighted Stein identity

$$
A^{*} \mathfrak{G}_{\boldsymbol{\beta}, C, A}^{(k+1)} A+\beta_{k}^{-1} C^{*} C=\mathfrak{G}_{\boldsymbol{\beta}, C, A}^{(k)}
$$

holds for all integers $k \geq 0$.

Proof. Indeed, since $\beta_{j} / \beta_{j+1} \leq M$ for all $j \geq 0$, we have

$$
\frac{\beta_{j}}{\beta_{j+k}} \leq M^{k} \quad \text { for all } k, j \geq 0
$$

and then it follows from (2.9), (3.1) and (3.2) that

$$
\begin{aligned}
\mathfrak{G}_{\boldsymbol{\beta}, C, A}^{(k)} & =\sum_{j=0}^{\infty} \frac{\beta_{j}}{\beta_{j+k}} \beta_{j}^{-1} A^{j *} C^{*} C A^{j} \\
& \leq M^{k} \sum_{j=0}^{\infty} \beta_{j}^{-1} A^{j *} C^{*} C A^{j}=M^{k} \cdot \mathcal{G}_{\boldsymbol{\beta}, C, A}, \\
\left\|\mathfrak{O}_{\boldsymbol{\beta}, C, A}^{(k)} x\right\|^{2} & =\left\langle\sum_{j=0}^{\infty} \frac{\beta_{j}^{2}}{\beta_{j+k}^{2}} \beta_{j}^{-1} A^{j *} C^{*} C A^{j} x, x\right\rangle \\
& \leq M^{2 k} \cdot\left\langle\mathcal{G}_{\boldsymbol{\beta}, C, A} x, x\right\rangle=M^{2 k} \cdot\left\|\mathcal{O}_{\boldsymbol{\beta}, C, A} x\right\|^{2}
\end{aligned}
$$

proving inequalities (3.5). Equality (3.6) follows immediately from power series representation $(\underline{3.2})$ for $\mathfrak{G}_{\boldsymbol{\beta}, C, A}^{(k)}$ and the similar one for $\mathfrak{G}_{\boldsymbol{\beta}, C, A}^{(k+1)}$.

As suggested by the Agler hereditary functional calculus as formulated by Ambrozie-Engliš-Müller [9], we introduce the operator

$$
B_{A}: X \mapsto A^{*} X A
$$

mapping $\mathcal{L}(\mathcal{X})$ into itself, and then view $\mathcal{G}_{\boldsymbol{\beta}, C, A}$ (at least formally) as being given by

$$
\mathcal{G}_{\boldsymbol{\beta}, C, A}=R_{\boldsymbol{\beta}}\left(B_{A}\right)\left[C^{*} C\right] .
$$

If $\rho(A)<1$ (where $\rho(A)$ denotes the spectral radius of $A$ ), (3.7) is precise; in general one can make this precise by interpreting (3.7) in the form

$$
\mathcal{G}_{\boldsymbol{\beta}, C, A}=\lim _{r \uparrow 1} R_{\boldsymbol{\beta}}\left(r B_{A}\right)\left[C^{*} C\right] .
$$


Remark 3.2. Given an operator $A \in \mathcal{L}(\mathcal{X})$ and a function $f(z)=\sum_{j=0}^{\infty} f_{j} z^{j}$ in the Wiener algebra $W^{+}$, i.e., the coefficients $f_{j}$ satisfy the summability condition

$$
\sum_{j=0}^{\infty}\left|f_{j}\right|<\infty
$$

the operatorial map

$$
f\left(B_{A}\right):=\sum_{j=0}^{\infty} f_{j} B_{A}^{j}: X \mapsto \sum_{j=0}^{\infty} f_{j} A^{* j} X A^{j}
$$

is well defined for any $X \in \mathcal{L}(\mathcal{X})$ subject to inequalities

$$
X \geq A^{*} X A \geq 0 .
$$

Indeed, by spectral theory, assumption (3.9) yields that $\left\|A^{* j} X A^{j}\right\| \leq\|X\|$ for $j \geq 0$. For $f$ in the Wiener algebra $W^{+}$, we see that $\sum_{j=0}^{\infty}\left|f_{j}\right|\left\|A^{* j} X A^{j}\right\|$ converges. We conclude that the sum defining $f\left(B_{A}\right)(X)$ is absolutely convergent in operator norm if $f \in W^{+}$and (3.9) holds.

Proposition 3.3. If $R_{\boldsymbol{\beta}}$ and $R_{\boldsymbol{\beta}, k}$ are defined as in (1.4) and (2.6), and if $\frac{1}{R_{\boldsymbol{\beta}}}$ belongs to $W^{+}$, then $\frac{R_{\boldsymbol{\beta}, k}}{R_{\boldsymbol{\beta}}}$ belongs to $W^{+}$for all $k \geq 1$.

Proof. Due to condition (1.9), the function

$$
g_{k}(z)=\sum_{\ell=1}^{k} \frac{1}{\beta_{k-\ell}} \cdot\left(\sum_{j=0}^{\infty} c_{\ell+j} z^{j}\right)
$$

belongs to $W_{+}$and moreover, $\left\|g_{k}\right\|_{W^{+}} \leq\left(\sum_{\ell=1}^{k} \beta_{k-\ell}^{-1}\right) \cdot\left\|\frac{1}{R_{\boldsymbol{\beta}}}\right\|_{W^{+}}$. Therefore, the order of summation in $(3.10)$ can be switched and we get, again making use of (1.9),

$$
\begin{aligned}
g_{k}(z) & =\sum_{j=0}^{\infty}\left(\sum_{\ell=1}^{k} \frac{c_{j+\ell}}{\beta_{k-\ell}}\right) z^{j}=-\sum_{j=0}^{\infty}\left(\sum_{\ell=0}^{j} \frac{c_{j-\ell}}{\beta_{k+\ell}}\right) z^{j} \\
& =-\left(\sum_{j=0}^{\infty} \frac{z^{j}}{\beta_{k+j}}\right) \cdot\left(\sum_{j=0}^{\infty} c_{j} z^{j}\right)=-\frac{R_{\boldsymbol{\beta}, k}(z)}{R_{\boldsymbol{\beta}}(z)}
\end{aligned}
$$

where the last equality holds due to (2.6) and (1.8). This completes the proof, since $g_{k}$ is in $W_{+}$. 
Making use of the reciprocal power series (1.8) let us introduce the operator map

$$
\Gamma_{\boldsymbol{\beta}, A}=\frac{1}{R_{\boldsymbol{\beta}}}\left(B_{A}\right): X \mapsto \sum_{j=0}^{\infty} c_{j} A^{* j} X A^{j},
$$

which, according to Remark 3.2, is well defined for any operator $X \in \mathcal{L}(\mathcal{X})$ subject to inequalities (3.9). We next use the power series (3.11) to define a family of operator maps

$$
\Gamma_{\boldsymbol{\beta}, A}^{(k)}=\frac{R_{\boldsymbol{\beta}, k}}{R_{\boldsymbol{\beta}}}\left(B_{A}\right): X \mapsto-\sum_{j=0}^{\infty}\left(\sum_{\ell=1}^{k} \frac{c_{j+\ell}}{\beta_{k-\ell}}\right) A^{* j} X A^{j}
$$

for all $k \geq 0$, which are again well defined for any operator $X \in \mathcal{L}(\mathcal{X})$ subject to inequalities (3.9), by Proposition 3.3 and Remark 3.2. Observe that $\Gamma_{\boldsymbol{\beta}, A}^{(0)}$ amounts to the identity mapping.

Proposition 3.4. Let us assume that conditions (1.7), (1.9) are in force and let $(C, A)$ be a $\boldsymbol{\beta}$-output-stable pair. Then the gramian $\mathcal{G}_{\boldsymbol{\beta}, C, A}$ is subject to relations

$$
\mathcal{G}_{\boldsymbol{\beta}, C, A} \geq A^{*} \mathcal{G}_{\boldsymbol{\beta}, C, A} A \geq 0, \quad \Gamma_{\boldsymbol{\beta}, A}\left[\mathcal{G}_{\boldsymbol{\beta}, C, A}\right]=C^{*} C
$$

and

$$
\Gamma_{\boldsymbol{\beta}, A}^{(k)}\left[\mathcal{G}_{\boldsymbol{\beta}, C, A}\right]=\mathfrak{G}_{\boldsymbol{\beta}, C, A}^{(k)} \geq 0 \quad \text { for all } \quad k \geq 1
$$

Proof. Since $\boldsymbol{\beta}$ is non-increasing (by the third condition in (1.7)), it follows from the power series representation (3.1) that

$$
\mathcal{G}_{\boldsymbol{\beta}, C, A}-A^{*} \mathcal{G}_{\boldsymbol{\beta}, C, A} A=C^{*} C+\sum_{j=1}^{\infty}\left(\beta_{j}^{-1}-\beta_{j-1}^{-1}\right) A^{* j} C^{*} C A^{j} \geq C^{*} C \geq 0,
$$

which proves the first statement in (3.14). Therefore, $\Gamma_{\boldsymbol{\beta}, A}\left[\mathcal{G}_{\boldsymbol{\beta}, C, A}\right]$ and $\Gamma_{\boldsymbol{\beta}, A}^{(k)}\left[\mathcal{G}_{\boldsymbol{\beta}, C, A}\right]$ are well-defined and the series (3.12), (3.13) (with $X=\mathcal{G}_{\boldsymbol{\beta}, C, A}$ ) converge absolutely against any $x \in \mathcal{X}$. Therefore, we can substitute the power series representation (3.1) for $\mathcal{G}_{\boldsymbol{\beta}, C, A}$ in (3.12) and then change the order of summation. This leads us to the second equality in (3.14):

$$
\begin{aligned}
\Gamma_{\boldsymbol{\beta}, A}\left[\mathcal{G}_{\boldsymbol{\beta}, C, A}\right] & =\sum_{j=0}^{\infty} c_{j} A^{* j}\left(\sum_{r=0}^{\infty} \beta_{r}^{-1} A^{* r} C^{*} C A^{r}\right) A^{j} \\
& =\sum_{\ell=0}^{\infty}\left(\sum_{j=0}^{\ell} c_{j} \beta_{\ell-j}^{-1}\right) A^{* \ell} C^{*} C A^{\ell}=C^{*} C
\end{aligned}
$$


where the last step follows from the recursion in (1.9). The same recursion implies

$$
\begin{aligned}
\sum_{r=0}^{j} \frac{1}{\beta_{j-r}} \cdot \sum_{\ell=1}^{k} \frac{c_{\ell+r}}{\beta_{k-\ell}} & =-\sum_{r=0}^{j} \frac{1}{\beta_{j-r}} \cdot \sum_{i=0}^{r} \frac{c_{i}}{\beta_{k+r-i}} \\
& =-\sum_{\ell=0}^{j} \frac{1}{\beta_{k+\ell}} \cdot \sum_{r=0}^{j-\ell} \frac{c_{r}}{\beta_{j-\ell-r}}=-\frac{1}{\beta_{k+j}}
\end{aligned}
$$

which together with (3.1), (3.13) and (3.12) leads us to

$$
\begin{aligned}
\Gamma_{\boldsymbol{\beta}, A}^{(k)}\left[\mathcal{G}_{\boldsymbol{\beta}, C, A}\right] & =-\sum_{j=0}^{\infty}\left(\sum_{\ell=1}^{k} \frac{c_{j+\ell}}{\beta_{k-\ell}}\right) A^{* j}\left(\sum_{r=0}^{\infty} \beta_{r}^{-1} A^{* r} C^{*} C A^{r}\right) A^{j} \\
& =-\sum_{j=0}^{\infty}\left(\sum_{r=0}^{j} \sum_{\ell=1}^{k} \frac{c_{\ell+r}}{\beta_{k-\ell} \beta_{j-r}}\right) A^{* j} C^{*} C A^{j} \\
& =\sum_{j=0}^{\infty} \beta_{j+k}^{-1} \cdot A^{* j} C^{*} C A^{j}=\mathfrak{G}_{\boldsymbol{\beta}, C, A}^{(k)}
\end{aligned}
$$

which verifies (3.15) and thus completes the proof.

Proposition 3.5. Let us assume that the weight sequence $\boldsymbol{\beta}$ meets conditions (1.7), (1.9) and let the operators $A, H \in \mathcal{L}(\mathcal{X})$ meet the conditions

$$
H \geq A^{*} H A \geq 0, \quad \Gamma_{\boldsymbol{\beta}, A}^{(k)}[H] \geq 0 \quad \text { for all } k \geq 1
$$

(the existence of operators $\Gamma_{\boldsymbol{\beta}, A}^{(k)}[H]$ is justified in Remark [3.2). Then

(1) There exists the strong limit

$$
\Delta_{A, H}=\lim _{k \rightarrow \infty} A^{* k} \Gamma_{\boldsymbol{\beta}, A}^{(k)}[H] A^{k} \geq 0 .
$$

(2) If in addition, $\Gamma_{\boldsymbol{\beta}, A}[H] \geq 0$, then the series below converges in the strong operator topology and satisfies

$$
\sum_{j=0}^{\infty} \beta_{j}^{-1} A^{* j} \Gamma_{\boldsymbol{\beta}, A}[H] A^{j}=H-\Delta_{A, H} .
$$

Proof. From definitions (1.4) and (2.7), it follows that for every $j \geq 0$,

$$
R_{\boldsymbol{\beta}, j}(z)=z R_{\boldsymbol{\beta}, j+1}(z)+\beta_{j}^{-1}
$$

Dividing both parts by $R_{\boldsymbol{\beta}}(z)$ and applying Agler hereditary functional calculus to the resulting identity and to the chosen operator $H$ (this can be done thanks to Remark 3.2 and Proposition 3.3) gives the operator equality

$$
\begin{aligned}
\Gamma_{\boldsymbol{\beta}, A}^{(j)}[H] & =B_{A} \Gamma_{\boldsymbol{\beta}, A}^{(j+1)}[H]+\beta_{j}^{-1} \Gamma_{\boldsymbol{\beta}, A}[H] \\
& =A^{*} \Gamma_{\boldsymbol{\beta}, A}^{(j+1)}[H] A+\beta_{j}^{-1} \Gamma_{\boldsymbol{\beta}, A}[H]
\end{aligned}
$$


where $\Gamma_{\boldsymbol{\beta}, A}^{(j)}[H]$ is simply $H$ for the case $j=0$. This in turn implies that

$$
A^{* j} \Gamma_{\boldsymbol{\beta}, A}^{(j)}[H] A^{j}-A^{* j+1} \Gamma_{\boldsymbol{\beta}, A}^{(j+1)}[H] A^{j+1}=\beta_{j}^{-1} A^{* j} \Gamma_{\boldsymbol{\beta}, A}[H] A^{j} \geq 0 .
$$

Therefore, the sequence of operators $A^{* k} \Gamma_{\boldsymbol{\beta}, A}^{(k)}[H] A^{k}$ is decreasing and bounded below and therefore has a strong limit (3.17).

Summing up equalities in (3.19) for $j=0, \ldots, k$ and taking into account that $\Gamma_{\boldsymbol{\beta}, A}^{(0)}[H]=H$ we get

$$
\sum_{j=0}^{k} \beta_{j}^{-1} \cdot A^{* j} \Gamma_{\boldsymbol{\beta}, A}[H] A^{j}=H-\left(A^{*}\right)^{k+1} \Gamma_{\boldsymbol{\beta}, A}^{(k+1)}[H] A^{k+1} .
$$

Letting $k \rightarrow \infty$ in the latter equality and making use of (3.17) we arrive at (3.18) thus completing the proof.

The following result gives connections between $\boldsymbol{\beta}$-output stability, observability gramians and solutions of associated Stein equalities and inequalities.

Theorem 3.6. Let us assume that the weight sequence $\boldsymbol{\beta}$ meets conditions (1.7), (1.9) and let $(C, A) \in \mathcal{L}(\mathcal{X}, \mathcal{Y}) \times \mathcal{L}(\mathcal{X})$ be an output pair. Then:

(1) The pair $(C, A)$ is $\boldsymbol{\beta}$-output-stable if and only if there is an $H \in \mathcal{L}(\mathcal{X})$ subject to inequalities (3.16) and the Stein inequality

$$
\Gamma_{\boldsymbol{\beta}, A}[H] \geq C^{*} C .
$$

(2) If $(C, A)$ is a $\boldsymbol{\beta}$-output-stable pair, then the gramian $H=\mathcal{G}_{\boldsymbol{\beta}, C, A}$ satisfies inequalities (3.16) and the Stein equality

$$
\Gamma_{\boldsymbol{\beta}, A}[H]=C^{*} C
$$

and is the minimal positive semidefinite solution of the system (3.16), (3.21).

Proof. Suppose first that $(C, A)$ is $\boldsymbol{\beta}$-output-stable. Then the infinite series in (3.1) converges strongly to the operator $H=\mathcal{G}_{\beta, C, A}$. By Proposition 3.4, $H$ satisfies relations (3.16), (3.22) and hence, also (3.21).

Conversely, suppose that (3.16), (3.21) hold for some $H \in \mathcal{L}(\mathcal{X})$. By Proposition 3.5, inequality (3.19) holds for all $k \geq 1$ which being combined with (3.21) gives

$$
\begin{aligned}
\sum_{j=0}^{k} \beta_{j}^{-1} \cdot A^{* j} C^{*} C A^{j} & \leq \sum_{j=0}^{k} \beta_{j}^{-1} \cdot A^{* j} \Gamma_{\boldsymbol{\beta}, A}[H] A^{j} \\
& =H-\left(A^{*}\right)^{k+1} \Gamma_{\boldsymbol{\beta}, A}^{(k+1)}[H] A^{k+1}
\end{aligned}
$$

for all $k \geq 1$. By letting $k \rightarrow \infty$ in (3.24) we conclude that the left hand side sum converges to a bounded positive semidefinite operator, which is $\mathcal{G}_{\boldsymbol{\beta}, C, A}$ by (3.1). Thus, passing to the limit in (3.24) as $k \rightarrow \infty$ gives $\mathcal{G}_{\beta, C, A} \leq$ $H-\Delta_{A, H}$ where $\Delta_{A, H} \geq 0$ is the limit defined in (3.17). Therefore, the 
pair $(C, A)$ is $\boldsymbol{\beta}$-output-stable and $\mathcal{G}_{\boldsymbol{\beta}, C, A}$ is indeed the minimal positive semidefinite solution to the system (3.16), (3.21).

Definition 3.7. Let us say that the operator $A \in \mathcal{L}(\mathcal{X})$ is $\boldsymbol{\beta}$-contractive if $A$ is a contraction and

$$
\Gamma_{\boldsymbol{\beta}, A}\left[I_{\mathcal{X}}\right]:=\sum_{j=0}^{\infty} c_{j} A^{* j} A^{j} \geq 0 .
$$

Let us say that $A$ is a $\boldsymbol{\beta}$-hypercontraction if in addition

$$
\Gamma_{\boldsymbol{\beta}, A}^{(k)}\left[I_{\mathcal{X}}\right]:=-\sum_{j=0}^{\infty}\left(\sum_{\ell=1}^{k} \frac{c_{j+\ell}}{\beta_{k-\ell}}\right) A^{* j} A^{j} \geq 0 \text { for all } k \geq 1 .
$$

Definition 3.8. A pair $(C, A) \in \mathcal{L}(\mathcal{X}, \mathcal{Y}) \times \mathcal{L}(\mathcal{X})$ will be called $\boldsymbol{\beta}$-contractive output pair if the inequalities (3.16), (3.21) hold with $H=I_{\mathcal{X}}$, that is, $A$ is $\boldsymbol{\beta}$-hypercontractive and

$$
\Gamma_{\boldsymbol{\beta}, A}\left[I_{\mathcal{X}}\right]:=\sum_{j=0}^{\infty} c_{j} A^{* j} A^{j} \geq C^{*} C .
$$

The pair $(C, A)$ will be called $\boldsymbol{\beta}$-isometric if $A$ is $\boldsymbol{\beta}$-hypercontractive and

$$
\Gamma_{\boldsymbol{\beta}, A}\left[I_{\mathcal{X}}\right]:=\sum_{j=0}^{\infty} c_{j} A^{* j} A^{j}=C^{*} C .
$$

Remark 3.9. Following the terminology from [73, 18], for $n$ a positive integer and $A$ an operator on a Hilbert space $\mathcal{X}$, we say that $A$ is an $n$ hypercontraction if $\Gamma_{k, A}\left[I_{\mathcal{X}}\right] \geq 0$ for $k=1$ and $k=n$, where in general, for $k$ an integer between 1 and $n$ we set

$$
\Gamma_{k, A}: H \mapsto\left(I-B_{A}\right)^{k}[H]=\sum_{j=0}^{k}(-1)^{j}\left(\begin{array}{c}
k \\
j
\end{array}\right) A^{* j} H A^{j} .
$$

If $A$ is an $n$-hypercontraction, it then follows automatically that $\Gamma_{k, A}\left[I_{\mathcal{X}}\right] \geq$ 0 for $1<k<n$ as well (see Lemma 4.3 in [18] or Lemma 1.1 in [73]). It is of interest to compare this notion to that of $\boldsymbol{\beta}$-hypercontraction as given in Definition 3.7 for the special choice of $\boldsymbol{\beta}$ with $\beta_{k}=\frac{k !(n-1) !}{(j+n-1) !}\left(\right.$ or $\boldsymbol{\beta}=\boldsymbol{\beta}_{\alpha}$ as in (1.5) with $\alpha=n): A$ is a $\boldsymbol{\beta}_{n}$-hypercontraction if $A$ is a contraction (so $\Gamma_{1, A}\left[I_{\mathcal{X}}\right] \geq 0$ ) and $\Gamma_{\boldsymbol{\beta}_{n}, A}\left[I_{\mathcal{X}}\right]=\Gamma_{n, A}\left[I_{\mathcal{X}}\right] \geq 0$ (so in fact $A$ is an $n$-hypercontraction) and also

$$
\Gamma_{\boldsymbol{\beta}_{n}, A}^{(k)}\left[I_{\mathcal{X}}\right] \geq 0 \quad \text { for all } k=1,2, \ldots
$$

We here check that this last condition (3.27) is automatic for an $n$ hypercontraction and hence the class of $n$-hypercontractions and the class of $\boldsymbol{\beta}_{n}$-hypercontractions are identical. To see this, we recall the notation for the resolvent and shifted resolvent:

$$
R_{\boldsymbol{\beta}_{n}}(z)=: R_{n}(z)=(1-z)^{-n}, \quad R_{\boldsymbol{\beta}_{n}, k}(z)=: R_{n, k}(z)
$$


and the formula for the shifted resolvent in terms of unshifted resolvents (see [18, Section 2]):

$$
R_{n, k}(z)=\sum_{\ell=1}^{n}\left(\begin{array}{c}
\ell+k-2 \\
\ell-1
\end{array}\right) R_{n-\ell+1}(z) .
$$

Hence specializing the formula (3.13) to the case $\boldsymbol{\beta}=\boldsymbol{\beta}_{n}$ gives us

$$
\begin{aligned}
\Gamma_{\boldsymbol{\beta}_{n}, A}^{(k)}\left(B_{A}\right) & =\frac{R_{n, k}}{R_{n}}\left(B_{A}\right) \\
& =\sum_{\ell=1}^{n}\left(\begin{array}{c}
\ell+k-2 \\
\ell-1
\end{array}\right) \frac{R_{n-\ell+1}}{R_{n}}\left(B_{A}\right) \\
& =\sum_{\ell=1}^{n}\left(\begin{array}{c}
\ell+k-2 \\
\ell-1
\end{array}\right) \Gamma_{\ell-1, A}\left(B_{A}\right) \\
& =\sum_{\ell=0}^{n-1}\left(\begin{array}{c}
\ell+k-1 \\
\ell
\end{array}\right) \Gamma_{\ell, A}\left(B_{A}\right) .
\end{aligned}
$$

Hence, if $A$ is an $n$-hypercontraction, then $\Gamma_{\ell, A}\left[I_{\mathcal{X}}\right] \geq 0$ for $\ell=0, \ldots, n-1$ and it follows that

$$
\Gamma_{\boldsymbol{\beta}_{n}, A}^{(k)}\left(B_{A}\right)\left[I_{\mathcal{X}}\right]=\sum_{\ell=0}^{n-1}\left(\begin{array}{c}
\ell+k-1 \\
\ell
\end{array}\right) \Gamma_{\ell, A}\left(B_{A}\right)\left[I_{\mathcal{X}}\right] \geq 0
$$

for all $k=1,2, \ldots$. We conclude that indeed any $n$-hypercontraction is also a $\boldsymbol{\beta}_{n}$-hypercontraction.

In addition we shall use the following standard terminology from systems theory. An output pair $(C, A)$ is called observable if the operator $\mathcal{G}_{1, C, \mathbf{A}}$ is injective. A pair $(C, A)$ is called exactly observable if $\mathcal{G}_{1, C, A}$ is bounded and is strictly positive definite. We note that the assumptions (1.7) on the sequence $\boldsymbol{\beta}$ imply that each term of the sequence is positive: $\beta_{j}>0$ for $j=0,1,2, \ldots$; this combined with the formula (3.1) enables one to see that $\mathcal{G}_{\boldsymbol{\beta}, C, A}$ is injective if and only if $\mathcal{G}_{1, C, A}$ is injective. In Proposition 4.5 below, we observe that the strict positivity of $\mathcal{G}_{1, C, A}$ implies the strict positivity of $\mathcal{G}_{\boldsymbol{\beta}, C, A}$. However the converse implication can fail in general (see [18, Proposition 5.7]. It therefore makes sense to introduce the following notion.

Definition 3.10. We will say that the pair $(C, A)$ is exactly $\boldsymbol{\beta}$-observable if the $\boldsymbol{\beta}$-gramian $\mathcal{G}_{\boldsymbol{\beta}, C, A}$ is bounded and is strictly positive definite.

Let us recall that an operator $A \in \mathcal{L}(\mathcal{X})$ is called strongly stable if $A^{k}$ tends to zero strongly: $\left\|A^{k} x\right\| \rightarrow 0$ as $k \rightarrow \infty$ for all $x \in \mathcal{X}$. We introduce the weighted analog of this notion for $\boldsymbol{\beta}$-hypercontraction.

Definition 3.11. A $\boldsymbol{\beta}$-hypercontractive operator $A \in \mathcal{L}(\mathcal{X})$ will be called $\boldsymbol{\beta}$-strongly stable if the limit (3.17) with $H=I_{\mathcal{X}}$ equals zero, i.e.,

$$
\Delta_{A, I}=\lim _{k \rightarrow \infty} A^{* k} \Gamma_{\boldsymbol{\beta}, A}^{(k)}[I] A^{k}=0,
$$


or, equivalently,

$$
-\lim _{k \rightarrow \infty} \sum_{j=0}^{\infty}\left(\sum_{\ell=1}^{k} \frac{c_{j+\ell}}{\beta_{k-\ell}}\right)\left\|A^{j+k} x\right\|^{2}=0 \quad \text { for all } \quad x \in \mathcal{X} .
$$

Remark 3.12. Observe that 1 -strong stability amounts to the usual strong stability. Indeed, if $\boldsymbol{\beta}=\mathbf{1}$, then $c_{0}=1, c_{1}=-1$ and $c_{j}=0$ for $j \geq 2$ (see (1.8)). Therefore, the $\operatorname{sum} \sum_{\ell=1}^{k} \frac{c_{j+\ell}}{\beta_{k-\ell}}$ equals to -1 if $j=0$ and it is equal to zero otherwise. Hence the double sum on the left of (3.28) amounts to the single term $\left\|A^{k} x\right\|^{2}$.

Lemma 3.13. (1) Suppose $(C, A)$ is a $\boldsymbol{\beta}$-contractive pair. Then $(C, A)$ is $\boldsymbol{\beta}$ output stable and $\mathcal{G}_{\boldsymbol{\beta}, C, A} \leq I$ so that $\mathcal{O}_{\boldsymbol{\beta}, C, A}: \mathcal{X} \rightarrow H_{\boldsymbol{\beta}}^{2}(\mathcal{Y})$ is a contraction.

(2) Suppose $(C, A)$ is a $\boldsymbol{\beta}$-isometric pair and $A$ is $\boldsymbol{\beta}$-strongly stable. Then $\mathcal{G}_{\boldsymbol{\beta}, C, A}=I$, the operator $\mathcal{O}_{\boldsymbol{\beta}, C, A}: \mathcal{X} \rightarrow H_{\boldsymbol{\beta}}^{2}(\mathcal{Y})$ is isometric and hence also the pair $(C, A)$ is exactly $\boldsymbol{\beta}$-observable.

Proof. The first statement follows from Theorem 3.6. To prove the second statement, we first observe that part (2) in Proposition 3.5 applies to $H=I$. Combining (3.25) and (3.18) (with $H=I$ ) now gives

$$
\sum_{j=0}^{\infty} \beta_{j}^{-1} A^{* j} C^{*} C A^{j}=I-\Delta_{A, I}
$$

Since $\Delta_{A, I}=0$ and since the series on the left equals $\mathcal{G}_{\boldsymbol{\beta}, C, A}$, we conclude $\mathcal{G}_{\boldsymbol{\beta}, C, A}=I$ which completes the proof.

\section{Observability-OPERATOR RANGE SPACES AND REPRODUCING KERNEL HILBERT SPACES}

Let $S_{\boldsymbol{\beta}}$ denote the shift operator on $H_{\boldsymbol{\beta}}^{2}(\mathcal{Y})$ defined as $S_{\boldsymbol{\beta}}: f(z) \rightarrow z f(z)$. Iterating the formula (1.6) for its adjoint gives

$$
S_{\boldsymbol{\beta}}^{* k} f=\sum_{j=0}^{\infty} \frac{\beta_{j+k}}{\beta_{j}} \cdot f_{j+k} z^{j} .
$$

Lemma 4.1. Let us assume that the weight sequence $\boldsymbol{\beta}$ meets conditions (1.7), (1.9). Let $E: H_{\boldsymbol{\beta}}^{2}(\mathcal{Y}) \rightarrow \mathcal{Y}$ be defined by $E f=f(0)$. Then

(1) $S_{\boldsymbol{\beta}}^{*}$ is strongly stable, i.e., $\lim _{k \rightarrow \infty}\left\|S_{\boldsymbol{\beta}}^{* k} f\right\|_{H_{\boldsymbol{\beta}}^{2}(\mathcal{Y})}=0$ for all $f \in H_{\boldsymbol{\beta}}^{2}(\mathcal{Y})$.

(2) The pair $\left(E, S_{\boldsymbol{\beta}}^{*}\right)$ is $\boldsymbol{\beta}$-output stable and the observability operator $\mathcal{O}_{\boldsymbol{\beta}, E, S_{\boldsymbol{\beta}}^{*}}$ equals the identity operator on $H_{\boldsymbol{\beta}}^{2}(\mathcal{Y})$.

(3) $S_{\boldsymbol{\beta}}^{*}$ is $\boldsymbol{\beta}$-hypercontractive and $\boldsymbol{\beta}$-strongly stable. Moreover,

$$
\Gamma_{\boldsymbol{\beta}, S_{\boldsymbol{\beta}}^{*}}\left[I_{H_{\boldsymbol{\beta}}^{2}(\mathcal{Y})}\right]=E^{*} E \quad \text { and } \quad \Gamma_{\boldsymbol{\beta}, S_{\boldsymbol{\beta}}^{*}}^{(k)}\left[I_{H_{\boldsymbol{\beta}}^{2}(\mathcal{Y})}\right]=\mathfrak{G}_{\boldsymbol{\beta}, E, S_{\boldsymbol{\beta}}^{*}}^{(k)}
$$


for all $k \geq 1$, or equivalently,

$$
\begin{gathered}
\sum_{j=1}^{\infty} c_{j}\left\|S_{\boldsymbol{\beta}}^{* j} f\right\|_{H_{\boldsymbol{\beta}}^{2}(\mathcal{Y})}^{2}=\|f(0)\|_{\mathcal{Y}}^{2}, \\
-\sum_{j=0}^{\infty}\left(\sum_{\ell=1}^{k} \frac{c_{j+\ell}}{\beta_{k-\ell}}\right)\left\|S_{\boldsymbol{\beta}}^{* j} f\right\|_{H_{\boldsymbol{\beta}}^{2}(\mathcal{Y})}^{2}=\sum_{j=0}^{\infty} \frac{\beta_{j}^{2}}{\beta_{k+j}}\left\|f_{j}\right\|_{\mathcal{Y}}^{2} \quad(k \geq 1)
\end{gathered}
$$

for all $f \in H_{\boldsymbol{\beta}}^{2}(\mathcal{Y})$, where $c_{j}$ 's are given in (1.9).

Proof. If $f(z)=\sum_{k=0}^{\infty} f_{k} z^{k}$ belongs to $H_{\boldsymbol{\beta}}^{2}(\mathcal{Y})$, then by (1.3),

$$
\sum_{j=k}^{\infty} \beta_{j}\left\|f_{j}\right\|_{\mathcal{Y}}^{2} \rightarrow 0 \quad \text { as } \quad k \rightarrow \infty
$$

and then it follows from (4.1) and (1.3) that

$$
\begin{aligned}
\left\|S_{\boldsymbol{\beta}}^{* k} f\right\|^{2}=\sum_{j=0}^{\infty} \frac{\beta_{j+k}^{2}}{\beta_{j}}\left\|f_{j+k}\right\|^{2} & \leq \sup _{j \geq 0} \frac{\beta_{j+k}}{\beta_{j}} \cdot \sum_{j=0}^{\infty} \beta_{j+k}\left\|f_{j+k}\right\|^{2} \\
& \leq \sum_{j=k}^{\infty} \beta_{j}\left\|f_{j}\right\|^{2} \rightarrow 0 \text { as } k \rightarrow \infty
\end{aligned}
$$

which proves the first statement. It follows from (4.1) that $E S_{\boldsymbol{\beta}}^{* j} f=\beta_{j} f_{j}$ and therefore,

$$
\mathcal{O}_{\boldsymbol{\beta}, E, S_{\boldsymbol{\beta}}^{*}} f=E R_{\boldsymbol{\beta}}\left(z S_{\boldsymbol{\beta}}^{*}\right) f=\sum_{j=0}^{\infty} \beta_{j}^{-1}\left(E S_{\boldsymbol{\beta}}^{* j} f\right) z^{j}=\sum_{j=0}^{\infty} f_{j} z^{j}=f
$$

which proves the second statement. Therefore $\mathcal{G}_{\boldsymbol{\beta}, E, S_{\boldsymbol{\beta}}^{*}}=I$ and hence, equalities (4.2) follow by Proposition 3.4. The useful more general identity

$$
\mathfrak{O}_{\boldsymbol{\beta}, E, S_{\boldsymbol{\beta}}^{*}}^{(k)} f=\sum_{j=0}^{\infty} \frac{\beta_{j}}{\beta_{k+j}} f_{j} z^{j}
$$

follows from the computation

$$
\mathfrak{O}_{\boldsymbol{\beta}, E, S_{\boldsymbol{\beta}}^{*}}^{(k)} f=\sum_{j=0}^{\infty} \frac{1}{\beta_{k+j}}\left(E S_{\boldsymbol{\beta}}^{* j} f\right) z^{j}=\sum_{j=0}^{\infty} \frac{\beta_{j}}{\beta_{k+j}} f_{j} z^{j} .
$$

Next we note that the first operator equality in (4.2) is equivalent to the quadratic-form identity

$$
\left\langle\Gamma_{\boldsymbol{\beta}, S_{\boldsymbol{\beta}}^{*}}\left[I_{H_{\boldsymbol{\beta}}^{2}(\mathcal{Y})}\right] f, f\right\rangle=\left\langle E^{*} E f, f\right\rangle_{H_{\boldsymbol{\beta}}^{2}(\mathcal{Y})}
$$


holding for all $f \in H_{\boldsymbol{\beta}}^{2}(\mathcal{Y})$ which in turn is equivalent to (4.3). To verify the equivalence of the second operator identity in (4.2) with the quadratic-form identity (4.4), note first that the equality

$$
\left\langle\Gamma_{\boldsymbol{\beta}, S_{\boldsymbol{\beta}}^{*}}^{(k)}\left[I_{H_{\boldsymbol{\beta}}^{2}(\mathcal{Y})}\right] f, f\right\rangle_{H_{\boldsymbol{\beta}}^{2}(\mathcal{Y})}=-\sum_{j=0}^{\infty}\left(\sum_{\ell=1}^{k} \frac{c_{j+\ell}}{\beta_{k-\ell}}\right)\left\|S_{\boldsymbol{\beta}}^{* j} f\right\|_{H_{\boldsymbol{\beta}}^{2}(\mathcal{Y})}^{2}
$$

is immediate from the definitions, while the identity

$$
\left\langle\mathfrak{G}_{\boldsymbol{\beta}, E, S_{\boldsymbol{\beta}}^{*}}^{(k)} f, f\right\rangle_{H_{\boldsymbol{\beta}}^{2}(\mathcal{Y})}=\sum_{j=0}^{\infty} \frac{\beta_{j}^{2}}{\beta_{k+j}}\left\|f_{j}\right\|_{\mathcal{Y}}^{2}
$$

follows from the computation

$$
\begin{aligned}
\left\langle\mathfrak{G}_{\boldsymbol{\beta}, E, S_{\boldsymbol{\beta}}^{*}}^{(k)} f, f\right\rangle_{H_{\boldsymbol{\beta}}^{2}(\mathcal{Y})} & =\left\|S_{\boldsymbol{\beta}}^{k} \mathfrak{O}_{\boldsymbol{\beta}, E, S_{\boldsymbol{\beta}}^{*}}^{(k)} f\right\|_{H_{\boldsymbol{\beta}}^{2}(\mathcal{Y})}^{2}(\operatorname{using}(\underline{3.3)})) \\
& =\left\|\sum_{j=0}^{\infty} \frac{\beta_{j}}{\beta_{k+j}} f_{j} z^{j+k}\right\|^{2}(\operatorname{using}(4.6)) \\
& =\sum_{j=0}^{\infty} \beta_{j+k} \cdot\left(\frac{\beta_{j}}{\beta_{k+j}}\right)^{2}\left\|f_{j}\right\|_{\mathcal{Y}}^{2} \\
& =\sum_{j=0}^{\infty} \frac{\beta_{j}^{2}}{\beta_{j+k}}\left\|f_{j}\right\|_{\mathcal{Y}}^{2}
\end{aligned}
$$

Combining (4.7) and (4.1) gives

$$
\begin{aligned}
\left\langle\mathfrak{G}_{\boldsymbol{\beta}, E, S_{\boldsymbol{\beta}}^{*}}^{(k)} S_{\boldsymbol{\beta}}^{* k} f, S_{\boldsymbol{\beta}}^{* k} f\right\rangle_{H_{\boldsymbol{\beta}}^{2}(\mathcal{Y})} & =\sum_{j=0}^{\infty} \frac{\beta_{j}^{2}}{\beta_{k+j}}\left\|\left(S_{\boldsymbol{\beta}}^{* k} f\right)_{j}\right\|_{\mathcal{Y}}^{2} \\
& =\sum_{j=0}^{\infty} \frac{\beta_{j}^{2}}{\beta_{k+j}}\left\|\frac{\beta_{j+k}}{\beta_{j}} f_{j+k}\right\|_{\mathcal{Y}}^{2} \\
& =\sum_{j=0}^{\infty} \beta_{j+k} \cdot\left\|f_{j+k}\right\|_{\mathcal{Y}}^{2}=\sum_{j=k}^{\infty} \beta_{j} \cdot\left\|f_{j}\right\|_{\mathcal{Y}}^{2} .
\end{aligned}
$$

This together with (4.5) and the second equality in (4.2) implies that

$$
\begin{aligned}
\left\langle S_{\boldsymbol{\beta}}^{k} \Gamma_{\boldsymbol{\beta}, S_{\boldsymbol{\beta}}^{*}}^{(k)}[I] S_{\boldsymbol{\beta}}^{* k} f, f\right\rangle_{H_{\boldsymbol{\beta}}^{2}(\mathcal{Y})} & =\left\langle\mathfrak{G}_{\boldsymbol{\beta}, E, S_{\boldsymbol{\beta}}^{*}}^{(k)} S_{\boldsymbol{\beta}}^{* k} f, S_{\boldsymbol{\beta}}^{* k} f\right\rangle_{H_{\boldsymbol{\beta}}^{2}(\mathcal{Y})} \\
& =\sum_{j=k}^{\infty} \beta_{j} \cdot\left\|f_{j}\right\|_{\mathcal{Y}}^{2} \rightarrow 0 \text { as } \quad k \rightarrow \infty .
\end{aligned}
$$

This finally verifies $\boldsymbol{\beta}$-strong stability of $S_{\boldsymbol{\beta}}$ and completes the proof.

Associated with a $\boldsymbol{\beta}$-output-stable pair $(C, A)$ is the range of the observability operator

$$
\operatorname{Ran} \mathcal{O}_{\boldsymbol{\beta}, C, A}=\left\{C R_{\boldsymbol{\beta}}(z A) x: x \in \mathcal{X}\right\}
$$


Theorem 4.2. Suppose that $(C, A)$ is a $\boldsymbol{\beta}$-output-stable pair. Then

(1) The intertwining relation

$$
S_{\boldsymbol{\beta}}^{*} \mathcal{O}_{\boldsymbol{\beta}, C, A}=\mathcal{O}_{\boldsymbol{\beta}, C, A} A
$$

holds and hence the linear manifold $\mathcal{M}=\operatorname{Ran} \mathcal{O}_{\boldsymbol{\beta}, C, A}$ is $S_{\boldsymbol{\beta}}^{*}$-invariant.

(2) If in addition, $(C, A)$ is exactly $\boldsymbol{\beta}$-observable, then the linear manifold $\mathcal{M}:=\operatorname{Ran} \mathcal{O}_{\boldsymbol{\beta}, C, A}$ given the lifted norm

$$
\left\|\mathcal{O}_{\boldsymbol{\beta}, C, A} x\right\|_{\mathcal{M}}^{2}=\left\langle\mathcal{G}_{\boldsymbol{\beta}, C, A} x, x\right\rangle_{\mathcal{X}},
$$

is isometrically included in $H_{\boldsymbol{\beta}}^{2}(\mathcal{Y})$ and is isometrically equal to the reproducing kernel Hilbert space with reproducing kernel

$$
K_{\boldsymbol{\beta}, C, A}(z, \zeta)=C R_{\boldsymbol{\beta}}(z A) \mathcal{G}_{\boldsymbol{\beta}, C, A}^{-1} R_{\boldsymbol{\beta}}(\zeta A)^{*} C^{*} .
$$

(3) Conversely, if $\mathcal{M}$ is a Hilbert space isometrically included in $H_{\boldsymbol{\beta}}^{2}(\mathcal{Y})$ which is invariant under $S_{\boldsymbol{\beta}}^{*}$, then there is a $\boldsymbol{\beta}$-output stable exactly $\boldsymbol{\beta}$-observable pair $(C, A)$ such that $\mathcal{M}=\mathcal{H}\left(K_{\boldsymbol{\beta}, C, A}\right)=\operatorname{Ran} \mathcal{O}_{\boldsymbol{\beta}, C, A}$ isometrically.

Proof. Making use of power series expansion (2.9) and of (1.6) we get (4.8):

$$
S_{\boldsymbol{\beta}}^{*} \mathcal{O}_{\boldsymbol{\beta}, C, A} x=S_{\boldsymbol{\beta}}^{*} \sum_{k=0}^{\infty} \beta_{k}^{-1}\left(C A^{k} x\right) z^{k}=\sum_{k=0}^{\infty} \beta_{k}^{-1}\left(C A^{k+1} x\right) z^{k}=\mathcal{O}_{\boldsymbol{\beta}, C, A} A x .
$$

The second statement follows from the definition (3.1) and general principles laid out in [25] (see also [27, 72, 18] for applications very close to the context here). To prove the last statement, observe that for $\mathcal{M}$ a Hilbert space isometrically included in $H_{\boldsymbol{\beta}}^{2}(\mathcal{Y})$, we may let $A=\left.S_{\boldsymbol{\beta}}^{*}\right|_{\mathcal{M}}$ and let $C$ be defined by $C f=f(0)$ for all $f \in \mathcal{M}$. In other words $C=\left.E\right|_{\mathcal{M}}$. Then the pair $(C, A)$ is $\boldsymbol{\beta}$-output stable and exactly $\boldsymbol{\beta}$-observable. It follows from part (2) in Lemma 4.1 that $\mathcal{M}=\operatorname{Ran} \mathcal{O}_{\beta, C, A}$.

Theorem 4.2 leads to the following operator-model theorem.

Theorem 4.3. Suppose that the Hilbert space operator $A \in \mathcal{L}(\mathcal{X})$ is a $\boldsymbol{\beta}$ strongly stable $\boldsymbol{\beta}$-hypercontraction. Let $\mathcal{Y}$ be a coefficient Hilbert space with $\operatorname{dim} \mathcal{Y}=\operatorname{rank} \Gamma_{\boldsymbol{\beta}, A}\left[I_{\mathcal{X}}\right]$. Then there is a subspace $\mathcal{N} \subset H_{\boldsymbol{\beta}}^{2}(\mathcal{Y})$ invariant under $S_{\boldsymbol{\beta}}^{*}$ so that $T$ is unitarily equivalent to $\left.S_{\boldsymbol{\beta}}^{*}\right|_{\mathcal{N}}$.

Proof. We choose $C \in \mathcal{L}(\mathcal{X}, \mathcal{Y})$ so that $C^{*} C=\Gamma_{\boldsymbol{\beta}, A}\left[I_{\mathcal{X}}\right]$. Then $(C, A$ is a $\boldsymbol{\beta}$-isometric pair. By Lemma 3.13, the assumption that $A$ is $\boldsymbol{\beta}$-strongly stable guarantees us that $\mathcal{O}_{\boldsymbol{\beta}, C, A}: \mathcal{X} \rightarrow H_{\boldsymbol{\beta}}^{2}(\mathcal{Y})$ is isometric. We set $\mathcal{N}=$ $\operatorname{Ran} \mathcal{O}_{\boldsymbol{\beta}, C, A} \subset H_{\boldsymbol{\beta}}^{2}(\mathcal{Y})$. Then the intertwining property (4.8) in Theorem 4.2 leads to the conclusion that $A$ is unitarily equivalent to $\left.S_{\boldsymbol{\beta}}^{*}\right|_{\mathcal{N}}$ via the unitary similarity transformation $\mathcal{O}_{\beta, C, A}: \mathcal{X} \rightarrow \mathcal{N}$.

Remark 4.4. As a consequence of Theorem 4.3 combined with part (1) of Lemma 4.1, we see that $\boldsymbol{\beta}$-strong stability of a $\boldsymbol{\beta}$-hypercontraction $A$ implies its strong stability in the usual sense $\left(\left\|A^{n} x\right\| \rightarrow 0\right.$ as $n \rightarrow \infty$ for 
each $x \in \mathcal{X}$ ). For the special case where $\boldsymbol{\beta}=\boldsymbol{\beta}_{n}$ for a positive integer $n$ as in Remark 3.9, it is known that strong stability of the $\boldsymbol{\beta}_{n}$-hypercontraction $A$ implies that $A$ is unitarily equivalent to the restriction of $S_{\boldsymbol{\beta}_{n}}^{*}$ to an invariant subspace $\mathcal{N} \subset H_{\boldsymbol{\beta}_{n}}^{2}(\mathcal{Y})=\mathcal{A}_{n}(\mathcal{Y})$ for a suitable coefficient Hilbert space $\mathcal{Y}$ as in Theorem 4.3, by results from [18] (see Theorem 5.3 part (2) there). When this is combined with part (3) of Lemma 4.1. we see that strong stability implies $\boldsymbol{\beta}_{n}$-strong stability for a $\boldsymbol{\beta}_{n}$-hypercontraction, and hence strong stability and $\boldsymbol{\beta}_{n^{-}}$strong stability are equivalent for $\boldsymbol{\beta}_{n^{-}}$ hypercontractions. We have not been able to determine at this time whether this equivalence holds for a general weight satisfying the standing hypothesis (1.7).

We record here the following fact which will be useful in the sequel.

Proposition 4.5. Suppose that the pair $(C, A)$ is $\boldsymbol{\beta}$-output-stable and exactly $\boldsymbol{\beta}$-observable (so $\mathfrak{G}_{\boldsymbol{\beta}, C, A}^{(0)}=\mathcal{G}_{\boldsymbol{\beta}, C, A}$ is strictly positive definite). Then it follows that $\mathfrak{G}_{\boldsymbol{\beta}, C, A}^{(k)}$ is strictly positive definite for $k=1,2,3, \ldots$.

Proof. The standing assumption (1.7) gives us that $\beta_{k+1} \leq \beta_{k}$, i.e., $\frac{1}{\beta_{k}} \leq$ $\frac{1}{\beta_{k+1}}$, for all $k=0,1,2, \ldots$. We then read off from the formula (3.2) that $\mathfrak{G}_{\boldsymbol{\beta}, C, A}^{(k)} \leq \mathfrak{G}_{\boldsymbol{\beta}, C, A}^{(k+1)}$, and in particular $\mathcal{G}_{\boldsymbol{\beta}, C, A}=\mathfrak{G}_{\boldsymbol{\beta}, C, A}^{(0)} \leq \mathfrak{G}_{\boldsymbol{\beta}, C, A}^{(k)}$ for all $k=1,2,3, \ldots$ It now follows that strict positivity of $\mathcal{G}_{\boldsymbol{\beta}, C, A}$ implies strict positivity of $\mathfrak{G}_{\boldsymbol{\beta}, C, A}^{(k)}$ as asserted.

\section{Functions $\Theta_{k}$ And Metric COnStraints}

In this section we will study the transfer functions $\Theta_{k}$ introduced by the realization formula (2.10) when the system operators $A, B_{k}, C, D_{k}$ satisfy certain additional metric constraints. By Proposition 3.1. for a $\boldsymbol{\beta}$-output stable pair $(C, A)$, the associated backward-shifted observability operators $\mathfrak{O}_{\boldsymbol{\beta}, C, A}^{(k)}$ are bounded for all $k \geq 0$ as operators from $\mathcal{X}$ into $H_{\boldsymbol{\beta}}^{2}(\mathcal{Y})$. In this case, the multiplication operator $M_{\Theta_{k}}$ given (according to (2.10)) by

$$
M_{\Theta_{k}}=\beta_{k}^{-1} \cdot D_{k}+S_{\boldsymbol{\beta}} \mathfrak{O}_{\boldsymbol{\beta}, C, A}^{(k+1)} B_{k}: \mathcal{U}_{k} \rightarrow H_{\boldsymbol{\beta}}^{2}(\mathcal{Y})
$$

is also bounded. Therefore, the output function $\widehat{y}$ in (2.8),

$$
\widehat{y}(z)=\mathcal{O}_{\boldsymbol{\beta}, C, A} x+\sum_{k=0}^{N} z^{k} \Theta_{k}(z) u_{k}
$$

belongs to $H_{\boldsymbol{\beta}}^{2}(\mathcal{Y})$ for every choice of $x \in \mathcal{X}$ and $u_{k} \in \mathcal{U}_{k}$ for each $N \geq 1$. We next impose some additional metric relations on $\left[\begin{array}{cc}A & B_{k} \\ C & D_{k}\end{array}\right]$, specifically one or 
more of the relations

$$
\begin{aligned}
& A^{*} \mathfrak{G}_{\boldsymbol{\beta}, C, A}^{(k+1)} B_{k}+\beta_{k}^{-1} \cdot C^{*} D_{k}=0, \\
& B_{k}^{*} \mathfrak{G}_{\boldsymbol{\beta}, C, A}^{(k+1)} B_{k}+\beta_{k}^{-1} \cdot D_{k}^{*} D_{k} \leq I_{\mathcal{U}_{k}}, \\
& B_{k}^{*} \mathfrak{G}_{\boldsymbol{\beta}, C, A}^{(k+1)} B_{k}+\beta_{k}^{-1} \cdot D_{k}^{*} D_{k}=I_{\mathcal{U}_{k}},
\end{aligned}
$$

and show how these lead to boundedness and orthogonality properties for the associated multiplication operator $M_{\Theta_{k}}$. Due to equality (3.6), it turns out that relations (5.2) and (5.3) are equivalent to the matrix inequality

$$
\left[\begin{array}{cc}
A^{*} & C^{*} \\
B_{k}^{*} & D_{k}^{*}
\end{array}\right]\left[\begin{array}{cc}
\mathfrak{G}_{\boldsymbol{\beta}, C, A}^{(k+1)} & 0 \\
0 & \beta_{k}^{-1} \cdot I_{\mathcal{Y}}
\end{array}\right]\left[\begin{array}{cc}
A & B_{k} \\
C & D_{k}
\end{array}\right] \leq\left[\begin{array}{cc}
\mathfrak{G}_{\boldsymbol{\beta}, C, A}^{(k)} & 0 \\
0 & I_{\mathcal{U}_{k}}
\end{array}\right],
$$

while the equalities (5.2) and (5.4) are equivalent to the matrix equality

$$
\left[\begin{array}{cc}
A^{*} & C^{*} \\
B_{k}^{*} & D_{k}^{*}
\end{array}\right]\left[\begin{array}{cc}
\mathfrak{G}_{\boldsymbol{\beta}, C, A}^{(k+1)} & 0 \\
0 & \beta_{k}^{-1} \cdot I_{\mathcal{Y}}
\end{array}\right]\left[\begin{array}{cc}
A & B_{k} \\
C & D_{k}
\end{array}\right]=\left[\begin{array}{cc}
\mathfrak{G}_{\boldsymbol{\beta}, C, A}^{(k)} & 0 \\
0 & I_{\mathcal{U}_{k}}
\end{array}\right] .
$$

The two latter conditions are of metric nature; they express the contractivity or isometric property of the colligation operator $U_{k}=\left[\begin{array}{cc}A & B_{k} \\ C & D_{k}\end{array}\right]$ with respect to certain weights.

Lemma 5.1. Let $(C, A)$ be a $\boldsymbol{\beta}$-output stable pair and let $\Theta_{k}$ be defined as in (2.10) for some integer $k \geq 0$ and operators $B_{k} \in \mathcal{L}\left(\mathcal{U}_{k}, \mathcal{X}\right)$ and $D_{k} \in \mathcal{L}\left(\mathcal{U}_{k}, \mathcal{Y}\right)$.

(1) If equality (5.2) holds, then

(a) $\mathcal{O}_{\boldsymbol{\beta}, C, A} x$ is orthogonal to $S_{\boldsymbol{\beta}}^{k} \Theta_{k} u$ for all $x \in \mathcal{X}$ and $u \in \mathcal{U}_{k}$.

(b) $S_{\boldsymbol{\beta}}^{k} \Theta_{k} u$ is orthogonal to $S_{\boldsymbol{\beta}}^{m} \Theta_{k} u^{\prime}$ for all $m>k$ and $u, u^{\prime} \in \mathcal{U}_{k}$.

(2) If (5.3) holds, then $S_{\boldsymbol{\beta}}^{k} M_{\Theta_{k}}$ is a contraction from $\mathcal{U}_{k}$ into $H_{\boldsymbol{\beta}}^{2}(\mathcal{Y})$.

(3) If (5.4) holds, then $S_{\boldsymbol{\beta}}^{k} M_{\Theta_{k}}$ is an isometry from $\mathcal{U}_{k}$ into $H_{\boldsymbol{\beta}}^{2}(\mathcal{Y})$.

(4) If both (5.2) and (5.3) hold, i.e., if (5.5) holds, then $S_{\boldsymbol{\beta}}^{k} M_{\Theta_{k}}$ is a contraction from $H^{2}\left(\mathcal{U}_{k}\right)$ into $H_{\boldsymbol{\beta}}^{2}(\mathcal{Y})$.

(5) If (5.2) and (5.4) hold, i.e., if (5.6) holds, then

$$
\left\|S_{\boldsymbol{\beta}}^{k} \Theta_{k} f\right\|_{H_{\boldsymbol{\beta}}^{2}(\mathcal{Y})}^{2}=\|f\|_{H^{2}\left(\mathcal{U}_{k}\right)}^{2}-\sum_{j=1}^{\infty}\left\|\left(I-S_{\boldsymbol{\beta}}^{*} S_{\boldsymbol{\beta}}\right)^{1 / 2} S_{\boldsymbol{\beta}}^{k} \Theta_{k} S_{\mathbf{1}}^{* j} f\right\|^{2}
$$

for every $f \in H^{2}\left(\mathcal{U}_{k}\right)$, and

$$
\begin{gathered}
\beta_{k}^{-1} I_{\mathcal{U}_{k}}-\Theta_{k}(z)^{*} \Theta_{k}(\zeta)=\beta_{k} B_{k}^{*} R_{\boldsymbol{\beta}, k}(z A)^{*} \mathfrak{G}_{\boldsymbol{\beta}, C, A}^{(k+1)} R_{\boldsymbol{\beta}, k}(\zeta A) B_{k} \\
-\bar{z} \zeta \beta_{k} B_{k}^{*} R_{\boldsymbol{\beta}, k+1}(z A)^{*} \mathfrak{G}_{\boldsymbol{\beta}, C, A}^{(k)} R_{\boldsymbol{\beta}, k+1}(\zeta A) B_{k}
\end{gathered}
$$

for all $z, \zeta \in \mathbb{D}$. 
Proof of (1): We first observe the power series expansion

$$
\Theta_{k}(z)=\beta_{k}^{-1} D_{k}+\sum_{j=0}^{\infty} \beta_{j+k+1}^{-1} C A^{j} B_{k} z^{j+1}
$$

which is an immediate consequence of formulas (2.10) and (2.7). We then make use of expansions (2.9), (5.9) and the definition of the inner product in $H_{\boldsymbol{\beta}}^{2}(\mathcal{Y})$ to prove part (a):

$$
\begin{aligned}
& \left\langle S_{\boldsymbol{\beta}}^{k} \Theta_{k} u, \mathcal{O}_{\boldsymbol{\beta}, C, A} x\right\rangle_{H_{\boldsymbol{\beta}}^{2}(\mathcal{Y})}=\beta_{k} \cdot\left\langle\beta_{k}^{-1} D_{k} u, \beta_{k}^{-1} C A^{k} x\right\rangle_{\mathcal{Y}} \\
& \quad+\sum_{j=0}^{\infty} \beta_{j+k+1} \cdot\left\langle\beta_{j+k+1}^{-1} C A^{j} B_{k} u, \beta_{j+k+1}^{-1} C A^{j+k+1} x\right\rangle_{\mathcal{Y}} \\
& =\left\langle\left(\beta_{k}^{-1} C^{*} D_{k}+A^{*}\left(\sum_{j=0}^{\infty} \beta_{j+k+1}^{-1} A^{* j} C^{*} C A^{j}\right) B_{k}\right) u, A^{k} x\right\rangle_{\mathcal{X}} \\
& =\left\langle\left(\beta_{k}^{-1} C^{*} D_{k}+A^{*} \mathfrak{G}_{\boldsymbol{\beta}, C, A}^{(k+1)} B_{k}\right) u, A^{k} x\right\rangle_{\mathcal{X}}=0
\end{aligned}
$$

where the two last equalities are justified by (3.2) and (5.2), respectively. Verification of part $(b)$ is quite similar: for $m>k$ we have

$$
\begin{aligned}
& \left\langle S_{\boldsymbol{\beta}}^{m} \Theta_{k} u^{\prime}, S_{\boldsymbol{\beta}}^{k} \Theta_{k} u\right\rangle_{H_{\boldsymbol{\beta}}^{2}(\mathcal{Y})}=\beta_{m} \cdot\left\langle\beta_{k}^{-1} D_{k} u^{\prime}, \beta_{m}^{-1} C A^{m-k-1} B_{k} u\right\rangle_{\mathcal{Y}} \\
& \quad+\sum_{j=0}^{\infty} \beta_{j+m+1} \cdot\left\langle\beta_{j+k+1}^{-1} C A^{j} B_{k} u^{\prime}, \beta_{j+m+1}^{-1} C A^{j+m-k} B_{k} u\right\rangle_{\mathcal{Y}} \\
& =\left\langle\left(\beta_{k}^{-1} C^{*} D_{k}+A^{*}\left(\sum_{j=0}^{\infty} \beta_{j+k+1}^{-1} A^{* j} C^{*} C A^{j}\right) B_{k}\right) u^{\prime}, A^{m-k-1} B_{k} u\right\rangle_{\mathcal{X}} \\
& =\left\langle\left(\beta_{k}^{-1} C^{*} D_{k}+A^{*} \mathfrak{G}_{\boldsymbol{\beta}, C, A}^{(k+1)} B_{k}\right) u^{\prime}, A^{m-k-1} B_{k} u\right\rangle_{\mathcal{X}}=0 .
\end{aligned}
$$

Proof of (2) and (3): According to (5.3),

$$
\beta_{k}^{-1} \cdot\left\|D_{k} u\right\|_{\mathcal{Y}}^{2}+\left\langle\mathfrak{G}_{\boldsymbol{\beta}, C, A}^{(k+1)} B_{k} u, B_{k} u\right\rangle_{\mathcal{X}} \leq\|u\|_{\mathcal{U}_{k}}^{2}
$$

for all $u \in \mathcal{U}_{k}$. We now may make use of (3.3) and (5.10) to get

$$
\begin{aligned}
\left\|S_{\boldsymbol{\beta}}^{k} \Theta_{k} u\right\|_{H_{\boldsymbol{\beta}}^{2}(\mathcal{Y})}^{2} & =\left\|\left(\beta_{k}^{-1} S_{\boldsymbol{\beta}}^{k} D_{k}+S_{\boldsymbol{\beta}}^{k+1} \mathfrak{O}_{\boldsymbol{\beta}, C, A}^{(k+1)} B_{k}\right) u\right\|_{H_{\boldsymbol{\beta}}^{2}(\mathcal{Y})}^{2} \\
& =\left\|\beta_{k}^{-1} S_{\boldsymbol{\beta}}^{k} D_{k} u\right\|_{H_{\boldsymbol{\beta}}^{2}(\mathcal{Y})}^{2}+\left\|S_{\boldsymbol{\beta}}^{k+1} \mathfrak{O}_{\boldsymbol{\beta}, C, A}^{(k+1)} B_{k} u\right\|_{H_{\boldsymbol{\beta}}^{2}(\mathcal{Y})}^{2} \\
& =\beta_{k}^{-1}\left\|D_{k} u\right\|_{\mathcal{Y}}^{2}+\left\langle\mathfrak{G}_{\boldsymbol{\beta}, C, A}^{(k+1)} B_{k} u, B_{k} u\right\rangle_{\mathcal{X}} \leq\|u\|_{\mathcal{U}_{k}}^{2} .
\end{aligned}
$$

Thus, $S_{\boldsymbol{\beta}}^{k} M_{\Theta_{k}}$ is a contraction from $\mathcal{U}_{k}$ to $H_{\boldsymbol{\beta}}^{2}(\mathcal{Y})$. In case (5.4) holds, then (5.10) holds with equality and part (3) follows. 
Proof of (4): Under the assumption that both (5.2) and (5.3) hold, we shall show that for any $\mathcal{U}_{k}$-valued polynomial $f(z)=\sum_{j=0}^{m} f_{j} z^{j}$,

$$
\left\|S_{\boldsymbol{\beta}}^{k} \Theta_{k} f\right\|_{H_{\boldsymbol{\beta}}^{2}(\mathcal{Y})}^{2} \leq\|f\|_{H^{2}\left(\mathcal{U}_{k}\right)}^{2}=\sum_{j=0}^{m}\left\|f_{j}\right\|_{\mathcal{U}_{k}}^{2} .
$$

Let $S_{1}^{*}$ be the operator of backward shift on $H^{2}\left(\mathcal{U}_{k}\right)$ so that for the polynomial $f$ as above, $\left(S_{1}^{*} f\right)(z)=\sum_{j=0}^{m-1} f_{j+1} z^{j}$. By statements $(1 \mathrm{~b})$ and $(2)$ of the lemma, we have

$$
\begin{aligned}
\left\|S_{\boldsymbol{\beta}}^{k} \Theta_{k} f\right\|^{2} & =\left\|\sum_{j=0}^{m} S_{\boldsymbol{\beta}}^{k+j} \Theta_{k} f_{j}\right\|^{2} \\
& =\left\|S_{\boldsymbol{\beta}}^{k} \Theta_{k} f_{0}\right\|^{2}+\left\|\sum_{j=1}^{m} S_{\boldsymbol{\beta}}^{k+j} \Theta_{k} f_{j}\right\|^{2}(\text { by }(1 \mathrm{~b})) \\
& \leq\left\|f_{0}\right\|^{2}+\left\|S_{\boldsymbol{\beta}}^{k+1} \sum_{j=0}^{m-1} S_{\boldsymbol{\beta}}^{j} \Theta_{k} f_{j+1}\right\|^{2} \\
& =\left\|f_{0}\right\|^{2}+\left\|S_{\boldsymbol{\beta}}^{k+1} \Theta_{k} S_{\mathbf{1}}^{*} f\right\|^{2} \\
& =\left\|f_{0}\right\|^{2}+\left\|S_{\boldsymbol{\beta}}^{k} \Theta_{k} S_{\mathbf{1}}^{*} f\right\|^{2}-\left\|\left(I-S_{\boldsymbol{\beta}}^{*} S_{\boldsymbol{\beta}}\right)^{\frac{1}{2}} S_{\boldsymbol{\beta}}^{k} \Theta_{k} S_{1}^{*} f\right\|^{2} .
\end{aligned}
$$

Replacing $f$ by $S_{1}^{* j} f$ in (5.12) gives

$$
\begin{aligned}
\left\|S_{\boldsymbol{\beta}}^{k} \Theta_{k} S_{\mathbf{1}}^{* j} f\right\|^{2} \leq\left\|f_{j}\right\|^{2} & +\left\|S_{\boldsymbol{\beta}}^{k} \Theta_{k}\left(S_{\mathbf{1}}^{*}\right)^{j+1} f\right\|^{2} \\
& -\left\|\left(I-S_{\boldsymbol{\beta}}^{*} S_{\boldsymbol{\beta}}\right)^{\frac{1}{2}} S_{\boldsymbol{\beta}}^{k} \Theta_{k}\left(S_{1}^{*}\right)^{j+1} f\right\|^{2}
\end{aligned}
$$

for $j=1, \ldots, m$. Iteration of the inequality (5.12) using (5.13) then gives

$$
\begin{aligned}
\left\|S_{\boldsymbol{\beta}}^{k} \Theta_{k} f\right\|_{H_{\boldsymbol{\beta}}^{2}(\mathcal{Y})}^{2} & \leq \sum_{j=0}^{m}\left\|f_{j}\right\|_{\mathcal{U}_{k}}^{2}-\sum_{j=1}^{m}\left\|\left(I-S_{\boldsymbol{\beta}}^{*} S_{\boldsymbol{\beta}}\right)^{\frac{1}{2}} S_{\boldsymbol{\beta}}^{k} \Theta_{k} S_{\mathbf{1}}^{* j} f\right\|^{2} \\
& \leq \sum_{j=0}^{m}\left\|f_{j}\right\|_{\mathcal{U}_{k}}^{2} .
\end{aligned}
$$

Letting $m \rightarrow \infty$ in (5.14) now implies the validity of (5.11) for every $f \in$ $H^{2}\left(\mathcal{U}_{k}\right)$ and the proof of (4) is now complete.

Proof of (5): In case (5.2) and (5.4) hold, then (5.12) holds with equality as well as in (5.11), (5.13), and (5.14). Equality (5.7) now follows by letting $m \rightarrow \infty$ in (5.14). 
It remains to verify the formula (5.8) under assumption (5.6). The identity (5.6) is equivalent to (3.6), (5.2) and (5.4). We use these relations to compute

$$
\begin{aligned}
& \beta_{k}^{-1} I_{\mathcal{U}_{k}}-\Theta_{k}(z)^{*} \Theta_{k}(\zeta) \\
& =\beta_{k}^{-1} I_{\mathcal{U}_{k}}-\left[\beta_{k}^{-1} D_{k}^{*}+\bar{z} B_{k}^{*} R_{\boldsymbol{\beta}, k+1}(z A)^{*} C^{*}\right]\left[\beta_{k}^{-1} D_{k}+\zeta C R_{\boldsymbol{\beta}, k+1}(\zeta A) B_{k}\right] \\
& =\beta_{k}^{-1} I_{\mathcal{U}_{k}}-\beta_{k}^{-2} D_{k}^{*} D_{k}-\bar{z} \beta_{k}^{-1} B_{k}^{*} R_{\boldsymbol{\beta}, k+1}(z A)^{*} C^{*} D_{k} \\
& \quad \quad-\zeta \beta_{k}^{-1} D_{k}^{*} C R_{\boldsymbol{\beta}, k+1}(\zeta A) B_{k}-\bar{z} \zeta B_{k}^{*} R_{\boldsymbol{\beta}, k+1}(z A)^{*} C^{*} C R_{\boldsymbol{\beta}, k+1}(\zeta A) B_{k} \\
& =\beta_{k}^{-1} B_{k}^{*} \mathfrak{G}_{\boldsymbol{\beta}, C, A}^{(k+1)} B_{k}+\bar{z} B_{k}^{*} R_{\boldsymbol{\beta}, k+1}(z A)^{*} A^{*} \mathfrak{G}_{\boldsymbol{\beta}, C, A}^{(k+1)} B_{k} \\
& \quad+\zeta B_{k}^{*} \mathfrak{G}_{\boldsymbol{\beta}, C, A}^{(k+1)} A R_{\boldsymbol{\beta}, k+1}(\zeta A) B_{k} \\
& \quad-\bar{z} \zeta \beta_{k} B_{k}^{*} R_{\boldsymbol{\beta}, k+1}(z A)^{*}\left(\mathfrak{G}_{\boldsymbol{\beta}, C, A}^{(k)}-A^{*} \mathfrak{G}_{\boldsymbol{\beta}, C, A}^{(k+1)} A\right) R_{\boldsymbol{\beta}, k+1}(\zeta A) B_{k}
\end{aligned}
$$

where we made use of (3.6), (5.2) and (5.4) in the last step. By making use next of the relation

$$
R_{\boldsymbol{\beta}, k}(z A)=\beta_{k}^{-1} I+z A R_{\boldsymbol{\beta}, k+1}(z A)
$$

we can continue the computation as

$$
\begin{aligned}
\beta_{k}^{-1} & I_{\mathcal{U}_{k}}-\Theta_{k}(z)^{*} \Theta_{k}(\zeta) \\
= & \beta_{k}^{-1} B_{k}^{*} \mathfrak{G}_{\boldsymbol{\beta}, C, A}^{(k+1)} B_{k}+B_{k}^{*}\left(R_{\boldsymbol{\beta}, k}(z A)^{*}-\beta_{k}^{-1} I\right) \mathfrak{G}_{\boldsymbol{\beta}, C, A}^{(k+1)} B_{k} \\
& +B_{k}^{*} \mathfrak{G}_{\boldsymbol{\beta}, C, A}^{(k+1)}\left(R_{\boldsymbol{\beta}, k}(\zeta A)-\beta_{k}^{-1} I\right) B_{k} \\
& +\beta_{k} B_{k}^{*}\left(R_{\boldsymbol{\beta}, k}(z A)^{*}-\beta_{k}^{-1} I\right) \mathfrak{G}_{\boldsymbol{\beta}, C, A}^{(k+1)}\left(R_{\boldsymbol{\beta}, k}(\zeta A)-\beta_{k}^{-1} I\right) B_{k} \\
& -\beta_{k} \bar{z} \zeta B_{k}^{*} R_{\boldsymbol{\beta}, k+1}(z A)^{*} \mathfrak{G}_{\boldsymbol{\beta}, C, A}^{(k)} R_{\boldsymbol{\beta}, k+1}(\zeta A) B_{k} \\
= & \beta_{k} B_{k}^{*} R_{\boldsymbol{\beta}, k}(z A)^{*} \mathfrak{G}_{\boldsymbol{\beta}, C, A}^{(k+1)} R_{\boldsymbol{\beta}, k}(\zeta A) B_{k} \\
& -\bar{z} \zeta \beta_{k} B_{k}^{*} R_{\boldsymbol{\beta}, k+1}(z A)^{*} \mathfrak{G}_{\boldsymbol{\beta}, C, A}^{(k)} R_{\boldsymbol{\beta}, k+1}(\zeta A) B_{k}
\end{aligned}
$$

verifying formula (5.8).

The following result is an immediate consequence of Lemma 5.1.

Corollary 5.2. Let us assume that the pair $(C, A)$ is $\boldsymbol{\beta}$-output stable and that relations (5.2), (5.3) hold for all $k \geq 0$. Then the representation (5.1) of the function $\widehat{y}$ is orthogonal in the metric of $H_{\boldsymbol{\beta}}^{2}(\mathcal{Y})$ and

$$
\|\widehat{y}\|_{H_{\boldsymbol{\beta}}^{2}(\mathcal{Y})}^{2}=\left\|\mathcal{O}_{\boldsymbol{\beta}, C, A} x\right\|^{2}+\sum_{k=0}^{\infty}\left\|\Theta_{k} u_{k}\right\|^{2} \leq\left\|\mathcal{G}_{\boldsymbol{\beta}, C, A}^{\frac{1}{2}} x\right\|_{\mathcal{X}}^{2}+\sum_{k=0}^{\infty}\left\|u_{k}\right\|_{\mathcal{U}_{k}}^{2} .
$$

If relations (5.3) hold with equalities for all $k \geq 0$, then equality holds in (5.15).

Observe that in case the pair $(C, A)$ is exactly $\boldsymbol{\beta}$-observable (so $\mathfrak{G}_{\boldsymbol{\beta}, C, A}^{(k)}$ is strictly positive definite for all $k \geq 0$ by Proposition 4.5), the inequality 
(5.5) can equivalently be expressed as $\|\Xi\| \leq 1$ where $\Xi:\left[\begin{array}{l}\mathcal{X} \\ \mathcal{U}_{k}\end{array}\right] \rightarrow\left[\begin{array}{l}\mathcal{X} \\ \mathcal{Y}\end{array}\right]$ is the operator given by

$$
\Xi:=\left[\begin{array}{cc}
\left(\mathfrak{G}_{\boldsymbol{\beta}, C, A}^{(k+1)}\right)^{1 / 2} & 0 \\
0 & \beta_{k}^{-\frac{1}{2}} I_{\mathcal{Y}}
\end{array}\right]\left[\begin{array}{cc}
A & B_{k} \\
C & D_{k}
\end{array}\right]\left[\begin{array}{cc}
\left(\mathfrak{G}_{\boldsymbol{\beta}, C, A}^{(k)}\right)^{-1 / 2} & 0 \\
0 & I_{\mathcal{U}_{k}}
\end{array}\right]
$$

An equivalent condition is that $\left\|\Xi^{*}\right\| \leq 1$ which in turn can be expressed as

$$
\left[\begin{array}{ll}
A & B_{k} \\
C & D_{k}
\end{array}\right]\left[\begin{array}{cc}
\left(\mathfrak{G}_{\boldsymbol{\beta}, C, A}^{(k)}\right)^{-1} & 0 \\
0 & I_{\mathcal{U}_{k}}
\end{array}\right]\left[\begin{array}{cc}
A^{*} & C^{*} \\
B_{k}^{*} & D_{k}^{*}
\end{array}\right] \leq\left[\begin{array}{cc}
\left(\mathfrak{G}_{\boldsymbol{\beta}, C, A}^{(k+1)}\right)^{-1} & 0 \\
0 & \beta_{k} I_{\mathcal{Y}}
\end{array}\right]
$$

Note that equality (5.6) means that the operator $\Xi$ is isometric. Of particular interest is the case where $\Xi$ is coisometric, i.e., where the colligation operator $U_{k}=\left[\begin{array}{cc}A & B_{k} \\ C & D_{k}\end{array}\right]$ is coisometric with respect to the weights indicated below:

$$
\left[\begin{array}{cc}
A & B_{k} \\
C & D_{k}
\end{array}\right]\left[\begin{array}{cc}
\left(\mathfrak{G}_{\boldsymbol{\beta}, C, A}^{(k)}\right)^{-1} & 0 \\
0 & I_{\mathcal{U}_{k}}
\end{array}\right]\left[\begin{array}{cc}
A^{*} & C^{*} \\
B_{k}^{*} & D_{k}^{*}
\end{array}\right]=\left[\begin{array}{cc}
\left(\mathfrak{G}_{\boldsymbol{\beta}, C, A}^{(k+1)}\right)^{-1} & 0 \\
0 & \beta_{k} I_{\mathcal{Y}}
\end{array}\right]
$$

Lemma 5.3. Let $(C, A)$ be an exactly $\boldsymbol{\beta}$-observable $\boldsymbol{\beta}$-output stable pair and let $\Theta_{k}$ be defined as in (2.10) for some operators $B_{k} \in \mathcal{L}\left(\mathcal{U}_{k}, \mathcal{X}\right)$ and $D_{k} \in \mathcal{L}\left(\mathcal{U}_{k}\right)$ subject to equality (5.17). Then

$$
\begin{gathered}
\beta_{k}^{-1} I_{\mathcal{Y}}-\Theta_{k}(z) \Theta_{k}(\zeta)^{*}=C R_{\boldsymbol{\beta}, k}(z A)\left(\mathfrak{G}_{\boldsymbol{\beta}, C, A}^{(k)}\right)^{-1} R_{\boldsymbol{\beta}, k}(\zeta A)^{*} C^{*} \\
-z \bar{\zeta} \cdot C R_{\boldsymbol{\beta}, k+1}(z A)\left(\mathfrak{G}_{\boldsymbol{\beta}, C, A}^{(k+1)}\right)^{-1} R_{\boldsymbol{\beta}, k+1}(\zeta A)^{*} C^{*}
\end{gathered}
$$

Proof. The proof parallels the verification of the identity (5.8) done above. The weighted-coisometry condition (5.17) gives us the set of equations

$$
\begin{aligned}
A\left(\mathfrak{G}_{\boldsymbol{\beta}, C, A}^{(k)}\right)^{-1} A^{*}+B_{k} B_{k}^{*} & =\left(\mathfrak{G}_{\boldsymbol{\beta}, C, A}^{(k+1)}\right)^{-1}, \\
C\left(\mathfrak{G}_{\boldsymbol{\beta}, C, A}^{(k)}\right)^{-1} A^{*}+D_{k} B_{k}^{*} & =0, \\
C\left(\mathfrak{G}_{\boldsymbol{\beta}, C, A}^{(k)}\right)^{-1} C^{*}+D_{k} D_{k}^{*} & =\beta_{k} I_{\mathcal{Y} .}
\end{aligned}
$$


We then compute:

$$
\begin{aligned}
\beta_{k}^{-1} & I_{\mathcal{Y}}-\Theta_{k}(z) \Theta_{k}(\zeta)^{*} \\
= & \beta_{k}^{-1} I_{\mathcal{Y}}-\left[\beta_{k}^{-1} D_{k}+z C R_{\boldsymbol{\beta}, k+1}(z A) B_{k}\right]\left[\beta_{k}^{-1} D_{k}^{*}+\bar{\zeta} B_{k}^{*} R_{\boldsymbol{\beta}, k+1}(\zeta A)^{*} C^{*}\right] \\
= & \beta_{k}^{-1} I_{\mathcal{Y}}-\beta_{k}^{-2} D_{k} D_{k}^{*}-z C R_{\boldsymbol{\beta}, k+1}(z A) \beta_{k}^{-1} B_{k} D_{k}^{*}-\bar{\zeta} \beta_{k}^{-1} D_{k} B_{k}^{*} R_{\boldsymbol{\beta}, k+1}(\zeta A)^{*} C^{*} \\
& -z \bar{\zeta} \cdot C R_{\boldsymbol{\beta}, k+1}(z A) B_{k} B_{k}^{*} R_{\boldsymbol{\beta}, k+1}(\zeta A)^{*} C^{*}(\text { by (5.19)}) \\
= & \beta_{k}^{-2} C\left(\mathfrak{G}_{\boldsymbol{\beta}, C, A}^{(k)}\right)^{-1} C^{*}+z \beta_{k}^{-1} C R_{\boldsymbol{\beta}, k+1}(z A) A\left(\mathfrak{G}_{\boldsymbol{\beta}, C, A}^{(k)}\right)^{-1} C^{*} \\
& +\bar{\zeta} \beta_{k}^{-1} C\left(\mathfrak{G}_{\boldsymbol{\beta}, C, A}^{(k)}\right)^{-1} A^{*} R_{\boldsymbol{\beta}, k+1}(\zeta A)^{*} C^{*} \\
& -z \bar{\zeta} \cdot C R_{\boldsymbol{\beta}, k+1}(z A)\left[\left(\mathfrak{G}_{\boldsymbol{\beta}, C, A}^{(k+1)}\right)^{-1}-A\left(\mathfrak{G}_{\boldsymbol{\beta}, C, A}^{(k)}\right)^{-1} A^{*}\right] R_{\boldsymbol{\beta}, k+1}(\zeta A)^{*} C^{*} \\
= & C\left(\beta_{k}^{-1} I_{\mathcal{X}}+z R_{\boldsymbol{\beta}, k+1}(z A) A\right)\left(\mathfrak{G}_{\boldsymbol{\beta}, C, A}^{(k)}\right)^{-1}\left(\beta_{k}^{-1} I_{\mathcal{X}}+\bar{\zeta} A^{*} R_{\boldsymbol{\beta}, k+1}(\zeta A)^{*}\right) C^{*} \\
& -z \bar{\zeta} \cdot C R_{\boldsymbol{\beta}, k+1}(z A)\left(\mathfrak{G}_{\boldsymbol{\beta}, C, A}^{(k+1)}\right)^{-1} R_{\boldsymbol{\beta}, k+1}(\zeta A)^{*} C^{*} \\
= & C R_{\boldsymbol{\beta}, k}(z A)\left(\mathfrak{G}_{\boldsymbol{\beta}, C, A}^{(k)}\right)^{-1} R_{\boldsymbol{\beta}, k}(\zeta A)^{*} C^{*} \\
& -z \bar{\zeta} \cdot C R_{\boldsymbol{\beta}, k+1}(z A)\left(\mathfrak{G}_{\boldsymbol{\beta}, C, A}^{(k+1)}\right)^{-1} R_{\boldsymbol{\beta}, k+1}(\zeta A)^{*} C^{*} .
\end{aligned}
$$

Remark 5.4. More generally, if $\Theta_{k}(z)$ is given by (2.10) and if we do not assume the weighted coisometry condition (5.17), then the decomposition (5.18) holds in the more general form

$$
\begin{array}{r}
\beta_{k}^{-1} I_{\mathcal{Y}}-\Theta_{k}(z) \Theta_{k}(\zeta)^{*}=C R_{\boldsymbol{\beta}, k}(z A)\left(\mathfrak{G}_{\boldsymbol{\beta}, C, A}^{(k)}\right)^{-1} R_{\boldsymbol{\beta}, k}(\zeta A)^{*} C^{*} \\
-z \bar{\zeta} \cdot C R_{\boldsymbol{\beta}, k+1}(z A)\left(\mathfrak{G}_{\boldsymbol{\beta}, C, A}^{(k+1)}\right)^{-1} R_{\boldsymbol{\beta}, k+1}(\zeta A)^{*} C^{*}+\Xi_{k}(z, \zeta)
\end{array}
$$

where the defect kernel $\Xi_{k}(z, \zeta)$ is given by

$$
\begin{aligned}
\Xi_{k}(z, \zeta)= & {\left[\begin{array}{ll}
z C R_{n, k}(z A) & \beta_{k}^{-1} I_{\mathcal{Y}}
\end{array}\right]\left(\left[\begin{array}{cc}
\left(\mathfrak{G}_{\boldsymbol{\beta}, C, A}^{(k+1)}\right)^{-1} & 0 \\
0 & \beta_{k} I_{\mathcal{Y}}
\end{array}\right]\right.} \\
& \left.-\left[\begin{array}{ll}
A & B_{k} \\
C & D_{k}
\end{array}\right]\left[\begin{array}{cc}
\left(\mathfrak{G}_{\boldsymbol{\beta}, C, A}^{(k)}\right)^{-1} & 0 \\
0 & I_{\mathcal{Y}}
\end{array}\right]\left[\begin{array}{cc}
A^{*} & C^{*} \\
B_{k}^{*} & D_{k}^{*}
\end{array}\right]\right) \cdot\left[\begin{array}{c}
\zeta R_{\boldsymbol{\beta}, k}(\zeta A)^{*} C^{*} \\
\beta_{k}^{-1} I_{\mathcal{Y}}
\end{array}\right] .
\end{aligned}
$$

Since equality (5.17) implies inequality (5.5), it follows that under assumption of Lemma 5.3, all the conclusions of parts (1), (2) and (4) in Lemma 5.1 are true. To have all conclusions true, we need the operator (5.16) to be unitary.

Lemma 5.5. Suppose that we are given an integer $k \geq 0$ and an exactly $\boldsymbol{\beta}$ observable $\boldsymbol{\beta}$-output-stable pair $(C, A) \in \mathcal{L}(\mathcal{X}, \mathcal{Y}) \times \mathcal{L}(\mathcal{X})$. Then there exist operators $B_{k} \in \mathcal{L}\left(\mathcal{U}_{k}, \mathcal{X}\right)$ and $D_{k} \in \mathcal{L}\left(\mathcal{U}_{k}, \mathcal{Y}\right)$ such that equalities (5.17) and 
(5.6) hold. Explicitly, such $B_{k}$ and $C_{k}$ are essentially uniquely determined by solving the Cholesky factorization problem:

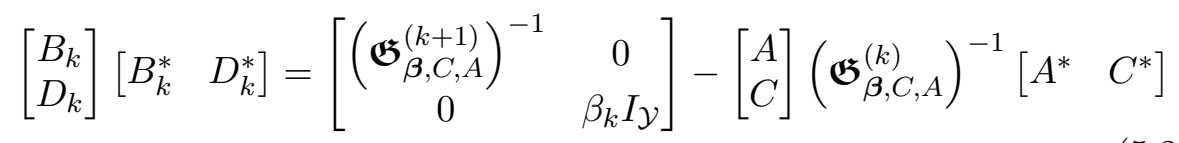

subject to the additional constraint that the coefficient space $\mathcal{U}_{k}$ be chosen so that $\left[\begin{array}{l}B_{k} \\ D_{k}\end{array}\right]: \mathcal{U}_{k} \rightarrow \mathcal{X} \oplus \mathcal{Y}$ is injective.

Proof. By Proposition 3.1, the weighted Stein identity (3.6) holds for each $k \geq 1$. Since $(C, A)$ is exactly observable, Proposition 4.5 assures us that the gramian $\mathfrak{G}_{\boldsymbol{\beta}, C, A}^{(k)}$ is strictly positive definite. It then follows from (3.6) that the operator

$$
\left[\begin{array}{c}
\left(\mathfrak{G}_{\boldsymbol{\beta}, C, A}^{(k+1)}\right)^{\frac{1}{2}} A\left(\mathfrak{G}_{\boldsymbol{\beta}, C, A}^{(k)}\right)^{-\frac{1}{2}} \\
\beta_{k}^{-\frac{1}{2}} C\left(\mathfrak{G}_{\boldsymbol{\beta}, C, A}^{(k)}\right)^{-\frac{1}{2}}
\end{array}\right]: \mathcal{X} \rightarrow \mathcal{X} \oplus \mathcal{Y}
$$

is an isometry. By extending this operator to a coisometric operator (5.16) we arrive at $B_{k}$ and $D_{k}$ solving (5.20). Further, extension of this operator to a unitary amounts to the additional restriction that $\left[\begin{array}{l}B_{k} \\ D_{k}\end{array}\right]$ be injective.

Remark 5.6. For the classical Hardy-space setting the general principle behind Lemma 5.5 is as follows: given a kernel on $\mathbb{D}$ with realization of the form $K(z, \zeta)=C(I-z A)^{-1}\left(I-\bar{\zeta} A^{*}\right)^{-1} C^{*}$ where the pair $(C, A)$ is isometric in the sense that $A^{*} A+C^{*} C=I$, one can produce a function $\Theta(z)$ with associated de Branges-Rovnyak kernel $K_{\Theta}(z, \zeta)$ equal to $K$ :

$$
\frac{I-\Theta(z) \Theta(\zeta)^{*}}{1-z \bar{\zeta}}=C(I-z A)^{-1}\left(I-\bar{\zeta} A^{*}\right)^{-1} C^{*} .
$$

Moreover, one can take $\Theta(z)$ to have the form $\Theta(z)=D+z C(I-z A)^{-1} B$ where $\left[\begin{array}{l}B \\ C\end{array}\right]$ is constructed as an injective solution of the Cholesky factorization problem

$$
\left[\begin{array}{l}
B \\
D
\end{array}\right]\left[\begin{array}{ll}
B^{*} & D^{*}
\end{array}\right]=\left[\begin{array}{ll}
I & 0 \\
0 & I
\end{array}\right]-\left[\begin{array}{l}
A \\
C
\end{array}\right]\left[\begin{array}{ll}
A^{*} & C^{*}
\end{array}\right] .
$$

This principle appears explicitly in [22, Section 3.1] for the indefinite metric setting and in [17, Theorem 1.3] for the Drury-Arveson-space multivariable setting.

The results of this section suggest that the following definition will be useful.

Definition 5.7. Suppose that

$$
U_{k}=\left[\begin{array}{ll}
A & B_{k} \\
C & D_{k}
\end{array}\right]:\left[\begin{array}{l}
\mathcal{X} \\
\mathcal{U}_{k}
\end{array}\right] \rightarrow\left[\begin{array}{l}
\mathcal{X} \\
\mathcal{Y}
\end{array}\right]
$$


is a colligation family with $A \boldsymbol{\beta}$-hypercontractive and $(C, A)$ exactly $\boldsymbol{\beta}$ observable. We then say that $\left\{U_{k}\right\}_{k \geq 0}$ is

(1) a $\boldsymbol{\beta}$-isometric colligation family if $U_{k}$ satisfies (5.6) for each $k$,

(2) a $\boldsymbol{\beta}$-coisometric colligation family if $U_{k}$ satisfies (5.17) for each $k$, and

(3) a $\boldsymbol{\beta}$-unitary colligation family if $U_{k}$ satisfies both (5.6) and (5.17) for each $k$.

The following corollary is an immediate consequence of Lemma 5.1 parts (1) and (3) together with formula (2.11). In its formulation we use the notation $H^{2}\left(\left\{\mathcal{U}_{k}\right\}_{k \geq 0}\right)$ for the "time-varying Hardy space" $\bigoplus_{k=0}^{\infty} z^{k} \mathcal{U}_{k}$ and we let $\ell_{\boldsymbol{\beta}}^{2}(\mathcal{Y})$ to denote the space of $\mathcal{Y}$-valued sequences $\{y(k)\}_{k \geq 0}$ with norm given by $\left\|\{y(k)\}_{k \geq 0}\right\|^{2}=\sum_{k=0}^{\infty} \boldsymbol{\beta}_{k}\|y\|_{\mathcal{Y}}^{2}$.

Corollary 5.8. Suppose that $\left\{U_{k}=\left[\begin{array}{cc}A & B_{k} \\ C & D_{k}\end{array}\right]\right\}$ is a $\boldsymbol{\beta}$-isometric family and let $\Sigma_{\boldsymbol{\beta}}$ be the associated time-varying linear system as in (2.1), and let $\left\{\Theta_{k}\right\}_{k \geq 0}$ be the associated transfer-function family as in (2.10). Then:

(1) The operator

$$
M_{\Theta}=\left[\begin{array}{llll}
M_{\Theta_{0}} & M_{\Theta_{1}} & M_{\Theta_{2}} & \cdot
\end{array}\right]: \bigoplus_{k=0}^{\infty} z^{k} u(k) \mapsto \sum_{k=0}^{\infty} \Theta_{k}(z) z^{k} u(k)
$$

is an isometry from $H^{2}\left(\left\{\mathcal{U}_{k}\right\}_{k \geq 0}\right)$ into $H_{\boldsymbol{\beta}}^{2}(\mathcal{Y})$.

(2) The input-output map $T_{\Sigma_{\boldsymbol{\beta}}}$ (2.5) acts as an isometry from $\bigoplus_{k=0}^{\infty} \mathcal{U}_{k}$ into $\ell_{\boldsymbol{\beta}}^{2}(\mathcal{Y})$.

Remark 5.9. To handle the case where $(C, A)$ is not necessarily exactly $\boldsymbol{\beta}$-observable, one can proceed as follows. We let $\mathcal{X}_{k}$ be the space $\mathcal{X}$ but with a new inner product

$$
\langle x, y\rangle_{\mathcal{X}_{k}}=\left\langle\mathfrak{G}_{\boldsymbol{\beta}, C, A}^{(k)} x, y\right\rangle .
$$

If $\mathfrak{G}_{\boldsymbol{\beta}, C, A}^{(k)}$ is not injective, we identify elements of self inner-product equal to 0 with the zero element of the space. We then complete $\mathcal{X}_{k}$ if necessary to arrive at a Hilbert space, still denoted as $\mathcal{X}_{k}$. Similarly, we let $\mathcal{Y}_{k}$ be the space $\mathcal{Y}$ but with new inner product

$$
\left\langle y, y^{\prime}\right\rangle \mathcal{Y}_{k}=\beta_{k}^{-1} \cdot\left\langle y, y^{\prime}\right\rangle \mathcal{Y}
$$

We let $A_{k}$ denote the operator $A$, but viewed as an operator from $\mathcal{X}_{k}$ to $\mathcal{X}_{k+1}$. Similarly we let $C_{k}$ denote the operator $C$ but viewed as an operator from $\mathcal{X}_{k}$ into $\mathcal{Y}_{k}$. Then the identity (3.6) tells us that the operator

$$
\left[\begin{array}{l}
A_{k} \\
C_{k}
\end{array}\right]: \mathcal{X}_{k} \rightarrow\left[\begin{array}{c}
\mathcal{X}_{k+1} \\
\mathcal{Y}_{k}
\end{array}\right]
$$

defined initially only on the image of $\mathcal{X} \oplus \mathcal{Y}$ in $\mathcal{X}_{k} \oplus \mathcal{Y}_{k}$, extends uniquely to a well-defined isometry. Thus $\mathcal{N}_{k}:=\operatorname{Ran}\left[\begin{array}{l}A_{k} \\ C_{k}\end{array}\right]$ is a closed subspace of $\mathcal{X}_{k} \oplus \mathcal{Y}_{k}$. 
We choose as coefficient space $\mathcal{U}_{k}$ a copy of the orthogonal complement

$$
\mathcal{U}_{k}=\left[\begin{array}{l}
\mathcal{X}_{k} \\
\mathcal{Y}_{k}
\end{array}\right] \ominus \mathcal{N}_{k}
$$

and let $\left[\begin{array}{c}B_{k} \\ D_{k}\end{array}\right]: \mathcal{U}_{k} \rightarrow\left[\begin{array}{c}\mathcal{X}_{k} \\ \mathcal{Y}_{k}\end{array}\right] \ominus \mathcal{N}_{k}$ be any convenient unitary identification map. In this way we arrive at a unitary colligation matrix

$$
\mathbf{U}_{k}=\left[\begin{array}{ll}
A_{k} & B_{k} \\
C_{k} & D_{k}
\end{array}\right]:\left[\begin{array}{l}
\mathcal{X}_{k} \\
\mathcal{U}_{k}
\end{array}\right] \rightarrow\left[\begin{array}{c}
\mathcal{X}_{k+1} \\
\mathcal{Y}_{k}
\end{array}\right]
$$

In case $B_{k}$ has range inside the image of $\mathcal{X}$ in $\mathcal{X}_{k}$, one can interpret the unitary property of $\mathbf{U}_{k}$ in terms of the original $\mathcal{X}$-inner product to arrive back at the relations (5.6). Even in the general case, the relations (5.6) still hold with proper interpretation. While this procedure is more general than that taken in Lemma 5.5, the construction via this procedure is less explicit.

\section{Beurling-Lax theorem for $H_{\boldsymbol{\beta}}^{2}(\mathcal{Y})$}

The classical Beurling-Lax theorem states that every shift-invariant closed subspace $\mathcal{M}$ of $H^{2}(\mathcal{Y})$ can be represented in the form $\mathcal{M}=\Theta \cdot H^{2}(\mathcal{U})$ for an auxiliary coefficient Hilbert space $\mathcal{U}$ and an inner $\mathcal{L}(\mathcal{U}, \mathcal{Y})$-valued function $\Theta$. In this section we present three analogues of the Beurling-Lax representation theorem for the weighted Hardy-space setting.

6.1. Shift-invariant subspaces contractively included in $H_{\boldsymbol{\beta}}^{2}(\mathcal{Y})$. Let us say that the Hilbert space $\mathcal{M}$ is contractively included in the Hilbert space $\mathcal{H}$ if $\mathcal{M} \subset \mathcal{H}$ as sets and moreover $\|m\|_{\mathcal{M}} \geq\|m\|_{\mathcal{H}}$ for all $m \in \mathcal{M}$. We also say that an $\mathcal{L}(\mathcal{U}, \mathcal{Y})$-valued function $\Theta$ is a contractive multiplier if the operator $M_{\Theta}: f(z) \mapsto \Theta(z) \cdot f(z)$ of multiplication by $\Theta$ defines a contractive operator from $H_{\boldsymbol{\beta}}^{2}(\mathcal{U})$ to $H_{\boldsymbol{\beta}}^{2}(\mathcal{Y})$.

Theorem 6.1. A Hilbert space $\mathcal{M}$ is such that

(1) $\mathcal{M}$ is contractively included in $H_{\boldsymbol{\beta}}^{2}(\mathcal{Y})$,

(2) $\mathcal{M}$ is $S_{\boldsymbol{\beta}}$-invariant: $S_{\boldsymbol{\beta}} \mathcal{M} \subset \mathcal{M}$,

(3) the operator $A=\left(\left.S_{\boldsymbol{\beta}}\right|_{\mathcal{M}}\right)^{*}$ is a $\boldsymbol{\beta}$-strongly stable $\boldsymbol{\beta}$-hypercontraction, if and only if there is a coefficient Hilbert space $\mathcal{U}$ and a contractive multiplier $\Theta$ so that

$$
\mathcal{M}=\Theta \cdot H_{\beta}^{2}(\mathcal{U})
$$

with lifted norm

$$
\|\Theta \cdot f\|_{\mathcal{M}}=\|Q f\|_{H_{\boldsymbol{\beta}}^{2}(\mathcal{U})}
$$

where $Q$ is the orthogonal projection onto $\left(\operatorname{Ker} M_{\Theta}\right)^{\perp}$. In this case $\mathcal{M}$ is itself a reproducing kernel Hilbert space with reproducing kernel given by

$$
K_{\mathcal{M}}(z, \zeta)=\Theta(z)\left(K_{\boldsymbol{\beta}}(z, \zeta) I_{\mathcal{U}}\right) \Theta(\zeta)^{*}, \quad(z, \zeta) \in \mathbb{D}^{2} .
$$


Proof. We first verify sufficiency. Suppose that $\mathcal{M}$ has the form (6.1) for a contractive multiplier $\Theta$ with $\mathcal{M}$-norm given by (6.2). Since $\left\|M_{\Theta}\right\| \leq 1$, it follows that

$$
\|\Theta f\|_{H_{\boldsymbol{\beta}}^{2}(\mathcal{Y})}=\left\|M_{\Theta} Q f\right\|_{H_{\boldsymbol{\beta}}^{2}(\mathcal{Y})} \leq\|Q f\|_{H_{\boldsymbol{\beta}}^{2}(\mathcal{U})}=\|\Theta f\|_{\mathcal{M}}
$$

i.e., (1) holds. Property (2) follows from the intertwining equality $S_{\boldsymbol{\beta}} M_{\Theta}=$ $M_{\Theta} S_{\boldsymbol{\beta}}$. The latter intertwining equality also implies $\left.M_{\Theta} S_{\boldsymbol{\beta}}\right|_{\operatorname{Ker} M_{\Theta}}=0$ which can be written equivalently in terms of the orthogonal projection $Q$ onto $\left(\operatorname{Ker} M_{\Theta}\right)^{\perp} \subset H_{\boldsymbol{\beta}}^{2}(\mathcal{U})$ as $Q S_{\boldsymbol{\beta}}(I-Q)=0$. Thus, we have

$$
Q S_{\boldsymbol{\beta}}=Q S_{\boldsymbol{\beta}} Q \quad \text { and } \quad S_{\boldsymbol{\beta}}^{*} Q=Q S_{\boldsymbol{\beta}}^{*} Q .
$$

Furthermore, for every $f, g \in H_{\boldsymbol{\beta}}^{2}(\mathcal{U})$, we have

$$
\begin{aligned}
\langle\Theta g, A \Theta f\rangle_{\mathcal{M}} & =\left\langle S_{\boldsymbol{\beta}} \Theta g, \Theta f\right\rangle_{\mathcal{M}}=\left\langle\Theta S_{\boldsymbol{\beta}} g, \Theta f\right\rangle_{\mathcal{M}}=\left\langle Q S_{\boldsymbol{\beta}} g, f\right\rangle_{H_{\boldsymbol{\beta}}^{2}(\mathcal{U})} \\
& =\left\langle Q S_{\boldsymbol{\beta}} Q g, f\right\rangle_{H_{\boldsymbol{\beta}}^{2}(\mathcal{U})}=\left\langle Q g, S_{\boldsymbol{\beta}}^{*} Q f\right\rangle_{H_{\boldsymbol{\beta}}^{2}(\mathcal{U})}=\left\langle\Theta g, \Theta S_{\boldsymbol{\beta}}^{*} Q f\right\rangle_{\mathcal{M}},
\end{aligned}
$$

which implies that $A: \Theta f \rightarrow \Theta S_{\boldsymbol{\beta}}^{*} Q f$. Iterating the latter formula gives

$$
A^{j}: \Theta f \rightarrow \Theta S_{\boldsymbol{\beta}}^{* j} Q f \quad \text { for } j \geq 0 .
$$

We have from (6.4), (6.5) and (6.2),

$$
\begin{aligned}
\left\langle\left(\Gamma_{\boldsymbol{\beta}, A}^{(k)}\left[I_{\mathcal{M}}\right]\right) \Theta f, \Theta f\right\rangle_{\mathcal{M}} & =\sum_{j=0}^{\infty}\left(-\sum_{\ell=1}^{k} \frac{c_{j+\ell}}{\beta_{k-\ell}}\right)\left\|A^{j} \Theta f\right\|_{\mathcal{M}}^{2} \\
& =\sum_{j=0}^{\infty}\left(-\sum_{\ell=1}^{k} \frac{c_{j+\ell}}{\beta_{k-\ell}}\right)\left\|S_{\boldsymbol{\beta}}^{* j} Q f\right\|_{H_{\boldsymbol{\beta}}^{2}(\mathcal{U})}^{2} \\
& =\left\langle\left(\Gamma_{\boldsymbol{\beta}, S_{\boldsymbol{\beta}}^{*}}^{(k)}\left[I_{H_{\boldsymbol{\beta}}^{2}(\mathcal{U})}\right]\right) Q f, Q f\right\rangle_{H_{\boldsymbol{\beta}}^{2}(\mathcal{U})}
\end{aligned}
$$

for $k=0,1,2, \ldots$, and also

$$
\begin{aligned}
\left\langle A_{j}^{* k} \Gamma_{\boldsymbol{\beta}, A}^{(k)}\left[I_{\mathcal{M}}\right] A^{k} \Theta f, \Theta f\right\rangle_{\mathcal{M}} & =\left\langle\Gamma_{\boldsymbol{\beta}, A}^{(k)}\left[I_{\mathcal{M}}\right] A^{k} \Theta f, A^{k} \Theta f\right\rangle_{\mathcal{M}} \\
& =\left\langle\Gamma_{\boldsymbol{\beta}, A}^{(k)}\left[I_{\mathcal{M}}\right] \Theta S_{\boldsymbol{\beta}}^{* k} Q f, \Theta S_{\boldsymbol{\beta}}^{* k} Q f\right\rangle_{\mathcal{M}} \\
& =\left\langle\left(\Gamma_{\boldsymbol{\beta}, S_{\boldsymbol{\beta}}^{*}}^{(k)}\left[I_{H_{\boldsymbol{\beta}}^{2}(\mathcal{U})}\right]\right) Q S_{\boldsymbol{\beta}}^{* k} Q f, Q S_{\boldsymbol{\beta}}^{* k} Q f\right\rangle_{H_{\boldsymbol{\beta}}^{2}(\mathcal{Y})} \\
& =\left\langle\left(\Gamma_{\boldsymbol{\beta}, S_{\boldsymbol{\beta}}^{*}}^{(k)}\left[I_{H_{\boldsymbol{\beta}}^{2}(\mathcal{U})}\right]\right) S_{\boldsymbol{\beta}}^{* k} Q f, S_{\boldsymbol{\beta}}^{* k} Q f\right\rangle_{H_{\boldsymbol{\beta}}^{2}(\mathcal{Y})}
\end{aligned}
$$

Since $S_{\boldsymbol{\beta}}^{*}$ is a $\boldsymbol{\beta}$-strongly stable $\boldsymbol{\beta}$-hypercontraction on $H_{\boldsymbol{\beta}}^{2}(\mathcal{Y})$, we conclude from the latter computations that

$$
\left\langle\left(\Gamma_{\boldsymbol{\beta}, A}^{(k)}\left[I_{\mathcal{M}}\right]\right) \Theta f, \Theta f\right\rangle_{\mathcal{M}}=\left\langle\left(\Gamma_{\boldsymbol{\beta}, S_{\boldsymbol{\beta}}^{*}}^{(k)}\left[I_{H_{\boldsymbol{\beta}}^{2}(\mathcal{U})}\right]\right) Q f, Q f\right\rangle_{H_{\boldsymbol{\beta}}^{2}(\mathcal{U})} \geq 0
$$

for $k \geq 0$, and that

$$
\lim _{k \rightarrow \infty}\left\langle A_{j}^{* k} \Gamma_{\boldsymbol{\beta}, A}^{(k)}\left[I_{\mathcal{M}}\right] A^{k} \Theta f, \Theta f\right\rangle_{\mathcal{M}}=0
$$


We conclude that $A$ is a $\boldsymbol{\beta}$-strongly stable $\boldsymbol{\beta}$-hypercontraction on $\mathcal{M}$ and thereby complete the proof of sufficiency.

Suppose now that the Hilbert space $\mathcal{M}$ satisfies conditions (1), (2), (3) in the statement of the theorem. Using hypothesis (2) we can define the operator $A:=\left(\left.S_{\boldsymbol{\beta}}\right|_{\mathcal{M}}\right)^{*}$ on $\mathcal{M}$ and since it is $\boldsymbol{\beta}$-hypercontractive by hypothesis (3), the operator $\Gamma_{\boldsymbol{\beta}, A}\left[I_{\mathcal{M}}\right]$ is positive semidefinite. Choose the coefficient Hilbert space $\mathcal{U}$ so that

$$
\operatorname{dim} \mathcal{U}=\operatorname{rank} \Gamma_{\boldsymbol{\beta}, A}\left[I_{\mathcal{M}}\right]
$$

and then choose the operator $C: \mathcal{M} \rightarrow \mathcal{U}$ so that $C^{*} C=\Gamma_{\boldsymbol{\beta}, A}\left[I_{\mathcal{M}}\right]$. Then $(C, A)$ is a $\boldsymbol{\beta}$-isometric pair and, since $A$ is $\boldsymbol{\beta}$-strongly stable by hypothesis (3), it follows that the observability operator $\mathcal{O}_{\boldsymbol{\beta}, C, A}: f \mapsto C R_{\boldsymbol{\beta}}(z A) f$ is an isometry from $\mathcal{M}$ into $H_{\boldsymbol{\beta}}^{2}(\mathcal{Y})$. By part (1) of Theorem 4.2, we have the intertwining equality (4.8). Taking adjoints in (4.8) then gives

$$
\mathcal{O}_{\boldsymbol{\beta}, C, A}^{*} S_{\boldsymbol{\beta}}=A^{*} \mathcal{O}_{\boldsymbol{\beta}, C, A}^{*}
$$

The inclusion map $\iota: \mathcal{M} \rightarrow H_{\boldsymbol{\beta}}^{2}(\mathcal{Y})$ is a contraction by hypothesis (1). Moreover, $\iota \circ A^{*}=S_{\boldsymbol{\beta}} \circ \iota: \mathcal{M} \rightarrow H_{\boldsymbol{\beta}}^{2}(\mathcal{Y})$. Therefore the operator

$$
R=\iota \circ \mathcal{O}_{\boldsymbol{\beta}, C, A}^{*}: H_{\boldsymbol{\beta}}^{2}(\mathcal{U}) \rightarrow H_{\boldsymbol{\beta}}^{2}(\mathcal{Y})
$$

is a contraction and

$$
R S_{\boldsymbol{\beta}}=\iota \circ \mathcal{O}_{\boldsymbol{\beta}, C, A}^{*} S_{\boldsymbol{\beta}}=\iota \circ A^{*} \mathcal{O}_{\boldsymbol{\beta}, C, A}^{*}=S_{\boldsymbol{\beta}} \circ \iota \circ \mathcal{O}_{\boldsymbol{\beta}, C, A}^{*}=S_{\boldsymbol{\beta}} R .
$$

Therefore (see [73]) $R$ is a multiplication operator, i.e., there is a contractive multiplier $\Theta$ so that $R=M_{\Theta}$. Since $\mathcal{O}_{\boldsymbol{\beta}, C, A}: \mathcal{M} \rightarrow H_{\boldsymbol{\beta}}^{2}(\mathcal{Y})$ is an isometry, it follows that $\operatorname{Ran} \mathcal{O}_{\boldsymbol{\beta}, C, A}^{*}=\mathcal{M}$ and also that $\mathcal{M}=\Theta \cdot H_{\boldsymbol{\beta}}^{2}(\mathcal{U})$ with $\mathcal{M}$-norm given by (6.2).

Finally, if $\mathcal{M}$ is given by (6.1) and (6.2) and if $f=\Theta \cdot g$ (with $g$ assumed to be in $\left(\operatorname{Ker} M_{\Theta}\right)^{\perp}$ ) is a generic element of $\mathcal{M}$, then we see from the liftednorm property transferred to inner products that

$$
\begin{aligned}
\left\langle f, \Theta k_{\boldsymbol{\beta}}(\cdot, \zeta) \Theta(\zeta)^{*} y\left\langle_{\mathcal{M}}\right.\right. & =\left\langle\Theta \cdot g, \Theta \cdot k_{\boldsymbol{\beta}}(\zeta, \zeta) u\right\rangle_{\mathcal{M}} \\
& =\left\langle g, k_{\boldsymbol{\beta}}(\cdot, \zeta) \Theta(\zeta)^{*} y\right\rangle_{H^{2}(\mathcal{U})} \\
& \left.=g(\zeta), \Theta(\zeta)^{*} y\right\rangle_{\mathcal{U}} \\
& =\langle\Theta(\zeta) g(\zeta), y\rangle_{\mathcal{Y}}=\langle f(\zeta), y\rangle_{\mathcal{Y}}
\end{aligned}
$$

and it follows that $\mathcal{M}=\mathcal{H}\left(K_{\mathcal{M}}\right)$ with $K_{\mathcal{M}}$ as in (6.3) as asserted.

Remark 6.2. Theorem 6.1 suggests the question as to how to characterize the contractive multipliers from $H_{\boldsymbol{\beta}}^{2}(\mathcal{U})$ into $H_{\boldsymbol{\beta}}^{2}(\mathcal{Y})$ in general. If the multiplication operator $M_{\Theta}: f \mapsto \Theta f$ is contractive from $H_{\boldsymbol{\beta}}^{2}(\mathcal{U})$ into $H_{\boldsymbol{\beta}}^{2}(\mathcal{Y})$, a standard reproducing-kernel-space computation shows that

$$
M_{\Theta}^{*}: K_{\boldsymbol{\beta}}(\cdot, \zeta) y \mapsto K_{\boldsymbol{\beta}}(\cdot, \zeta) \Theta(\zeta)^{*} y
$$


for each $\zeta \in \mathbb{D}$ and $y \in \mathcal{Y}$ from which it follows that the associated kernel

$$
L(z, \zeta):=\left(I-\Theta(z) \Theta(\zeta)^{*}\right) K_{\boldsymbol{\beta}}(z, \zeta)
$$

is a positive kernel on $\mathbb{D} \times \mathbb{D}$. By looking at the diagonal entries of this kernel we see that $\Theta(z) \Theta(z)^{*} \leq I$ for all $z \in \mathbb{D}$. Furthermore, by noting the action of $M_{\Theta}$ on constant vectors $u \in H_{\boldsymbol{\beta}}^{2}(\mathcal{U})$, we see that $\Theta$ is analytic. Conversely, it can be shown that the converse holds: any contractive analytic operatorvalued function $\Theta$ on $\mathbb{D}$ induces a contractive multiplier from $H_{\boldsymbol{\beta}}^{2}(\mathcal{U})$ into $H_{\boldsymbol{\beta}}^{2}(\mathcal{Y})$. The following more general formulation was suggested to us by the referee.

Theorem 6.3. Let $\boldsymbol{\beta}=\left\{\beta_{k}\right\}_{k \geq 0}$ be any positive non-increasing weight sequence such that $\beta_{k}^{1 / k} \rightarrow 1$ as $k \rightarrow \infty$. Let $\mathcal{U}$ and $\mathcal{Y}$ be auxiliary Hilbert spaces and let $\Theta$ be an $\mathcal{L}(\mathcal{U}, \mathcal{Y})$-valued function on the open unit disk $\mathbb{D}$. Then $\Theta$ is a contractive multiplier from $H_{\boldsymbol{\beta}}^{2}(\mathcal{U})$ into $H_{\boldsymbol{\beta}}^{2}(\mathcal{Y})$ if and only if $\Theta$ is analytic with $\|\Theta(z)\| \leq 1$ for $z \in \mathbb{D}$.

Proof. The necessity direction follows by the same argument as sketched before the statement of the theorem.

Conversely assume that $\Theta$ is analytic with contractive values on $\mathbb{D}$. By the argument sketched above, we see that $\Theta$ is a contractive multiplier if and only if the associated kernel $L(\underline{6.6})$ is a positive kernel on $\mathbb{D}$. The kernel $L$ factors in the form

$$
L(z, \zeta)=\frac{I_{\mathcal{Y}}-\Theta(z) \Theta(\zeta)^{*}}{1-z \bar{\zeta}} \cdot(1-z \bar{\zeta}) K_{\boldsymbol{\beta}}(z, \zeta)
$$

The first factor $\frac{I y-\Theta(z) \Theta(\zeta)^{*}}{1-z \bar{\zeta}}$ is a positive kernel on $\mathbb{D}$ since $\Theta$ is a contractive multiplier from $H^{2}(\mathcal{U})$ into $H^{2}(\mathcal{Y})$. The second factor $(1-z \bar{\zeta}) K_{\boldsymbol{\beta}}(z, \zeta)$ is a positive kernel on $\mathbb{D}$ since the shift operator $S_{\boldsymbol{\beta}}$ is a contraction on $H^{2}(\boldsymbol{\beta})$ (here we use that the weight sequence $\boldsymbol{\beta}$ is non-increasing). By the Schur theorem about Schur products of positive semidefinite matrices, it follows that the associated kernel $L$ is a positive kernel since it is the product of two positive kernels (see e.g. [11, Section 1.8]). Note that the application of the Schur theorem is fine as long as one of the kernels is scalar-valued. It now follows that indeed $\Theta$ is a contractive multiplier from $H_{\boldsymbol{\beta}}^{2}(\mathcal{U})$ into $H_{\boldsymbol{\beta}}^{2}(\mathcal{Y})$.

We note that this result sharpens the result of Giselsson-Olofsson [47, Proposition 4.1].

Remark 6.4. It is of interest to consider Theorem 6.1 for the case condition (1) is strengthened to $\left(1^{\prime}\right) \mathcal{M}$ is isometrically contained in $H_{\boldsymbol{\beta}}^{2}(\mathcal{Y})$. Unlike the classical Hardy space case $\left(\beta_{j}=1\right.$ for $\left.j=0,1,2, \ldots\right)$, condition (3) in Theorem 6.1 is not automatic and it may not be the case that $\mathcal{M}=\Theta \cdot H_{\boldsymbol{\beta}}^{2}(\mathcal{U})$ with $\Theta$ equal to a partially isometric multiplier; see Theorem 4.1 in [47] for a 
related result. For more detailed discussion of the case where $\beta_{j}=\frac{j !(n-1) !}{(n+j-1) !}$ for a positive integer $n$, see Remark 7.4 in [18].

6.2. Isometric representations of $S_{\beta}$-invariant subspaces via inner function families. In this section we obtain a finer representation for $S_{\boldsymbol{\beta}^{-}}$ invariant subspaces using a generalization of inner functions which we call an inner family (see Definition 6.8 below).

We start with a general observation. If the subspace $\mathcal{M} \subset H_{\boldsymbol{\beta}}^{2}(\mathcal{Y})$ is $S_{\boldsymbol{\beta}}$-invariant, then $\mathcal{M}^{\perp}$ is $S_{\boldsymbol{\beta}}^{*}$-invariant and, by part (3) of Theorem 4.2. we may find a $\boldsymbol{\beta}$-output stable exactly $\boldsymbol{\beta}$-observable pair $(C, A)$ so that $\mathcal{M}^{\perp}=\operatorname{Ran} \mathcal{O}_{\beta, C, A}$; in fact, we may take $(C, A)$ to be the model output pair $(C, A)=\left(\left.E\right|_{\mathcal{M}^{\perp}},\left.S_{\boldsymbol{\beta}}^{*}\right|_{\mathcal{M}^{\perp}}\right)$, and $\mathcal{O}_{\boldsymbol{\beta}, C, A}$ amounts to the inclusion map of $\mathcal{M}^{\perp}$ into $H_{\boldsymbol{\beta}}^{2}(\mathcal{Y})$.

Since $\left\|\mathcal{O}_{\boldsymbol{\beta}, C, A} x\right\|_{H_{\boldsymbol{\beta}}^{2}(\mathcal{Y})}^{2}=\left\langle\mathcal{G}_{\boldsymbol{\beta}, C, A} x, x\right\rangle_{\mathcal{X}}$ for every $x \in \mathcal{X}$, it follows that $\mathcal{M}^{\perp}$ is a reproducing kernel Hilbert space with reproducing kernel

$$
K_{\mathcal{M}^{\perp}}(z, \zeta)=C R_{\boldsymbol{\beta}}(z A) \mathcal{G}_{\boldsymbol{\beta}, C, A}^{-1} R_{\boldsymbol{\beta}}(\zeta A)^{*} C^{*} .
$$

It then follows that $\mathcal{M}=\left(\mathcal{M}^{\perp}\right)^{\perp}$ has reproducing kernel

$$
K_{\mathcal{M}}(z, \zeta)=K_{\boldsymbol{\beta}}(z, \zeta) \cdot I_{\mathcal{Y}}-C R_{\boldsymbol{\beta}}(z A) \mathcal{G}_{\boldsymbol{\beta}, C, A}^{-1} R_{\boldsymbol{\beta}}(\zeta A)^{*} C^{*}
$$

Since

$$
\bigcap_{k \geq 0} S_{\boldsymbol{\beta}}^{k} \mathcal{M} \subset \bigcap_{k \geq 0} S_{\boldsymbol{\beta}}^{k} H_{\boldsymbol{\beta}}^{2}(\mathcal{Y})=\{0\}
$$

we can decompose $\mathcal{M}$ into the orthogonal sum

$$
\mathcal{M}=\bigoplus_{k=0}^{\infty}\left(S_{\boldsymbol{\beta}}^{k} \mathcal{M} \ominus S_{\boldsymbol{\beta}}^{k+1} \mathcal{M}\right)
$$

To compute the reproducing kernel for the subspace $S_{\boldsymbol{\beta}}^{k} \mathcal{M} \ominus S_{\boldsymbol{\beta}}^{k+1} \mathcal{M}$, we first characterize the space $\left(S_{\boldsymbol{\beta}}^{k} \mathcal{M}\right)^{\perp}$ in terms of the shifted observability operator $\mathfrak{O}_{\boldsymbol{\beta}, C, A}^{(k)}$ defined in (3.2).

Proposition 6.5. The space $\left(S_{\boldsymbol{\beta}}^{k} \mathcal{M}\right)^{\perp}$ is characterized as

$$
\left(S_{\boldsymbol{\beta}}^{k} \mathcal{M}\right)^{\perp}=\left(\bigoplus_{j=0}^{k-1} S_{\boldsymbol{\beta}}^{j} \mathcal{Y}\right) \bigoplus S_{\boldsymbol{\beta}}^{k} \operatorname{Ran} \mathfrak{O}_{\boldsymbol{\beta}, C, A}^{(k)}
$$

where we identify the first term with the subspace of polynomials of degree at most $k-1$ in $H_{\boldsymbol{\beta}}^{2}(\mathcal{Y})$.

Proof. We wish to characterize all functions $f(z)=\sum_{j=0}^{\infty} f_{j} z^{j}$ which are orthogonal to $S_{\boldsymbol{\beta}}^{k} \mathcal{M}$ in $H_{\boldsymbol{\beta}}^{2}(\mathcal{Y})$. We may write $f(z)=p(z)+z^{k} \widetilde{f}(z)$ where 
$p(z)=\sum_{j=0}^{k-1} f_{j} z^{j}$. Clearly polynomials of degree at most $k-1$ are orthogonal to $S_{\boldsymbol{\beta}}^{k} \mathcal{M}$, so it suffices to characterize which functions of the form $z^{k} \widetilde{f}(z)$ are orthogonal to $S_{\boldsymbol{\beta}}^{k} \mathcal{M}$. To this end, observe that $S_{\boldsymbol{\beta}}^{k} \tilde{f}$ is orthogonal to $S_{\boldsymbol{\beta}}^{k} \mathcal{M}$ if and only if the function $\left(S_{\boldsymbol{\beta}}^{k}\right)^{*} S_{\boldsymbol{\beta}}^{k} \tilde{f}$ belongs to $\mathcal{M}^{\perp}=\operatorname{Ran} \mathcal{O}_{\boldsymbol{\beta}, C, A}$. It follows from the formula (4.1) that

$$
\left(S_{\boldsymbol{\beta}}^{k}\right)^{*} S_{\boldsymbol{\beta}}^{k}: \sum_{j=0}^{\infty} \widetilde{f}_{j} z^{j} \mapsto \sum_{j=0}^{\infty} \frac{\beta_{j+k}}{\beta_{j}} \widetilde{f}_{j} z^{j}
$$

We thus conclude that $S_{\boldsymbol{\beta}}^{k} \widetilde{f}$ is orthogonal to $S_{\boldsymbol{\beta}}^{k} \mathcal{M}$ if and only if there exists a vector $x \in \mathcal{X}$ such that

$$
\sum_{j=0}^{\infty} \frac{\beta_{j+k}}{\beta_{j}} \widetilde{f}_{j} z^{j}=C R_{\boldsymbol{\beta}}(z A) x=\sum_{j=0}^{\infty}\left(\beta_{j}^{-1} \cdot C A^{j} x\right) z^{j} .
$$

Equating the corresponding Taylor coefficients gives

$$
\widetilde{f}_{j}=\beta_{j+k}^{-1} \cdot C A^{j} x \quad \text { for all } \quad j \geq 0
$$

and therefore,

$$
\widetilde{f}(z)=\sum_{j=0}^{\infty} \widetilde{f}_{j} z^{j}=\sum_{j=0}^{\infty}\left(\beta_{j+k}^{-1} \cdot C A^{j} x\right) z^{j}=\mathfrak{O}_{\boldsymbol{\beta}, C, A}^{(k)} x .
$$

Thus, $\tilde{f} \in \operatorname{Ran} \mathfrak{O}_{\boldsymbol{\beta}, C, A}^{(k)}$. As the analysis is necessary and sufficient, the result follows.

With this result in hand, it is straightforward to derive the kernel function for the space $S_{\boldsymbol{\beta}}^{k} \mathcal{M}$ with respect to the metric inherited from $H_{\boldsymbol{\beta}}^{2}(\mathcal{Y})$.

Proposition 6.6. Let $\mathcal{M}$ be a closed shift-invariant subspace of $H_{\boldsymbol{\beta}}^{2}(\mathcal{Y})$ with reproducing kernel given by (6.7). Then the reproducing kernel functions for $S_{\boldsymbol{\beta}}^{k} \mathcal{M}$ and $S_{\boldsymbol{\beta}}^{k} \mathcal{M} \ominus S_{\boldsymbol{\beta}}^{k+1} \mathcal{M}$ are given by

$$
\begin{array}{r}
K_{S_{\boldsymbol{\beta}}^{k \mathcal{M}}}(z, \zeta)=z^{k} \bar{\zeta}^{k}\left(R_{\boldsymbol{\beta}, k}(z \bar{\zeta}) I_{\mathcal{Y}}-C R_{\boldsymbol{\beta}, k}(z A)\left(\mathfrak{G}_{\boldsymbol{\beta}, C, A}^{(k)}\right)^{-1} R_{\boldsymbol{\beta}, k}(\zeta A)^{*} C^{*}\right) \\
K_{S_{\boldsymbol{\beta}}^{k} \mathcal{M} \ominus S_{\boldsymbol{\beta}}^{k+1} \mathcal{M}}(z, \zeta)=z^{k} \bar{\zeta}^{k}\left(\beta_{k}^{-1} I_{\mathcal{Y}}-C R_{\boldsymbol{\beta}, k}(z A)\left(\mathfrak{G}_{\boldsymbol{\beta}, C, A}^{(k)}\right)^{-1} R_{\boldsymbol{\beta}, k}(\zeta A)^{*} C^{*}\right. \\
\left.+z \bar{\zeta} C R_{\boldsymbol{\beta}, k+1}(z A)\left(\mathfrak{G}_{\boldsymbol{\beta}, C, A}^{(k+1)}\right)^{-1} R_{\boldsymbol{\beta}, k+1}(\zeta A)^{*} C^{*}\right) .
\end{array}
$$


Proof. We first derive the kernel $\mathfrak{K}$ for the space $S_{\boldsymbol{\beta}}^{k} \operatorname{Ran} \mathfrak{O}_{\boldsymbol{\beta}, C, A}^{(k)}$ (with inner product induced by $\left.H_{\boldsymbol{\beta}}^{2}(\mathcal{Y})\right)$. By (3.3),

$$
\left\|S_{\boldsymbol{\beta}}^{k} \mathfrak{D}_{\boldsymbol{\beta}, C, A}^{(k)} x\right\|_{H_{\boldsymbol{\beta}}^{2}(\mathcal{Y})}^{2}=\left\langle\mathfrak{G}_{\boldsymbol{\beta}, C, A}^{(k)} x, x\right\rangle_{\mathcal{X}},
$$

and thus by the general principle from [25], it follows that the reproducing kernel for $S_{\boldsymbol{\beta}}^{k} \operatorname{Ran} \mathfrak{O}_{\boldsymbol{\beta}, C, A}^{(k)}$ is given by

$$
\mathfrak{K}_{k}(z, \zeta)=z^{k} \bar{\zeta}^{k} C R_{\boldsymbol{\beta}, k}(z A)\left(\mathfrak{G}_{\boldsymbol{\beta}, C, A}^{(k)}\right)^{-1} R_{\boldsymbol{\beta}, k}(\zeta A)^{*} C^{*} .
$$

From the formula $(6.9)$ for $\left(S_{\boldsymbol{\beta}} \mathcal{M}\right)^{\perp}$, we deduce that

$$
\begin{aligned}
S_{\boldsymbol{\beta}}^{k} \mathcal{M} & =\left(\bigoplus_{j=0}^{k-1} S_{\boldsymbol{\beta}}^{j} \mathcal{Y}\right)^{\perp} \bigcap\left(S_{\boldsymbol{\beta}}^{k} \operatorname{Ran} \mathfrak{D}_{\boldsymbol{\beta}, C, A}^{(k)}\right)^{\perp} \\
& =S_{\boldsymbol{\beta}}^{k} H_{\boldsymbol{\beta}}^{2}(\mathcal{Y}) \ominus S_{\boldsymbol{\beta}}^{k} \operatorname{Ran} \mathfrak{D}_{\boldsymbol{\beta}, C, A}^{(k)} .
\end{aligned}
$$

Since the reproducing kernel of the subspace $S_{\boldsymbol{\beta}}^{j} \mathcal{Y}$ of $H_{\boldsymbol{\beta}}^{2}(\mathcal{Y})$ is $z^{j} \bar{\zeta}^{j} \beta_{j}^{-1} I_{\mathcal{Y}}$, we deduce that $S_{\boldsymbol{\beta}}^{k} H_{\boldsymbol{\beta}}^{2}(\mathcal{Y})=H_{\boldsymbol{\beta}}^{2}(\mathcal{Y}) \ominus\left(\bigoplus_{j=0}^{k-1} S_{\boldsymbol{\beta}}^{j} \mathcal{Y}\right)$ has reproducing kernel

$$
\begin{aligned}
K_{S_{\boldsymbol{\beta}}^{k} H_{\boldsymbol{\beta}}^{2}(\mathcal{Y})}(z, \zeta) & =\left(K_{\boldsymbol{\beta}}(z, \zeta)-\sum_{j=0}^{k-1} \beta_{j}^{-1} z^{j} \bar{\zeta}^{j}\right) I_{\mathcal{Y}} \\
& =\left(R_{\boldsymbol{\beta}}(z \bar{\zeta})-\sum_{j=0}^{k-1} \beta_{j}^{-1} z^{j} \bar{\zeta}^{j}\right) I_{\mathcal{Y}}=z^{k} \bar{\zeta}^{k} R_{\boldsymbol{\beta}, k}(z \bar{\zeta}) I_{\mathcal{Y}} .
\end{aligned}
$$

Hence from (6.13) we deduce that

$$
K_{S_{\boldsymbol{\beta}}^{k} \mathcal{M}}(z, \zeta)=K_{S_{\boldsymbol{\beta}}^{k} H_{\boldsymbol{\beta}}^{2}(\mathcal{Y})}(z, \zeta)-\mathfrak{K}_{k}(z, \zeta)
$$

and formula (6.10) follows from (6.12). Finally, since

$$
K_{S_{\boldsymbol{\beta}}^{k} H_{\boldsymbol{\beta}}^{2}(\mathcal{Y})}(z, \zeta)-K_{S_{\boldsymbol{\beta}}^{k+1} H_{\boldsymbol{\beta}}^{2}(\mathcal{Y})}(z, \zeta)=z^{k} \bar{\zeta}^{k} \beta_{k}^{-1} I_{\mathcal{Y}},
$$

we have from the two latter equalities

$$
\begin{aligned}
K_{S_{\boldsymbol{\beta}}^{k} \mathcal{M} \ominus S_{\boldsymbol{\beta}}^{k+1} \mathcal{M}}(z, \zeta)= & K_{S_{\boldsymbol{\beta}}^{k} \mathcal{M}}(z, \zeta)-K_{S_{\boldsymbol{\beta}}^{k+1} \mathcal{M}}(z, \zeta) \\
= & K_{S_{\boldsymbol{\beta}}^{k} H_{\boldsymbol{\beta}}^{2}(\mathcal{Y})}(z, \zeta)-\mathfrak{K}_{k}(z, \zeta) \\
& \quad-K_{S_{\boldsymbol{\beta}}^{k+1} H_{\boldsymbol{\beta}}^{2}(\mathcal{Y})}(z, \zeta)+\mathfrak{K}_{k+1}(z, \zeta) \\
= & z^{k} \bar{\zeta}^{k} \beta_{k}^{-1} I_{\mathcal{Y}}-\mathfrak{K}_{k}(z, \zeta)+\mathfrak{K}_{k+1}(z, \zeta)
\end{aligned}
$$

which implies (6.11) due to (6.12). 
Lemma 6.7. Given an integer $k \geq 1$ and an exactly $\boldsymbol{\beta}$-observable $\boldsymbol{\beta}$-output stable pair $(C, A)$, construct operators $B_{k} \in \mathcal{L}\left(\mathcal{U}_{k}, \mathcal{X}\right)$ and $D_{k} \in \mathcal{L}\left(\mathcal{U}_{k}, \mathcal{Y}\right)$ as in Lemma 5.5 and let $\Theta_{k}$ be the associated function given by (2.10). Then the kernel (6.11) can be factored as

$$
K_{S_{\boldsymbol{\beta}}^{k} \mathcal{M} \ominus S_{\boldsymbol{\beta}}^{k+1} \mathcal{M}}(z, \zeta)=z^{k} \bar{\zeta}^{k} \Theta_{k}(z) \Theta_{k}(\zeta)^{*} .
$$

Proof. By Lemma 5.3, identity (5.18) holds. Multiplying both parts of (5.18) by $z^{k} \bar{\zeta}^{k}$ and combining the obtained equality with (6.11) we get (6.14).

Definition 6.8. In what follows, we will use the symbol $\bigvee$ for the closed linear span. A family of operator-valued functions $\left\{\Theta_{k}: \mathbb{D} \rightarrow \mathcal{L}\left(\mathcal{U}_{k}, \mathcal{Y}\right)\right\}_{k=0}^{\infty}$ will be said to be a $\boldsymbol{\beta}$-inner function family if, for each $k \geq 0$, we have:

(1) $M_{\Theta_{k}}: S_{1}^{k} \mathcal{U}_{k} \rightarrow H_{\boldsymbol{\beta}}^{2}(\mathcal{Y})$ is isometric,

(2) $M_{\Theta_{k}}\left(S_{1}^{k} \mathcal{U}_{k}\right)$ is orthogonal (in $\left.H_{\boldsymbol{\beta}}^{2}(\mathcal{Y})\right)$ to $\bigvee_{\ell>k} M_{\Theta_{\ell}} S_{\mathbf{1}}^{\ell} \mathcal{U}_{\ell}$,

(3) $S_{\boldsymbol{\beta}}^{k+1} M_{\Theta_{k}} \mathcal{U}_{k} \subset \bigvee_{\ell>k} S_{\boldsymbol{\beta}}^{\ell} M_{\Theta_{\ell}} \mathcal{U}_{\ell}$

An equivalent operator-theoretic characterization of the $\boldsymbol{\beta}$-inner function family property is:

$\left(1^{\prime}\right)$ The multiplication operator

$$
M_{\Theta}=\left[\begin{array}{llll}
M_{\Theta_{0}} & M_{\Theta_{1}} & M_{\Theta_{2}} & \cdots
\end{array}\right]: \bigoplus_{k=0}^{\infty} S_{1}^{k} \mathcal{U}_{k} \rightarrow H_{\boldsymbol{\beta}}^{2}(\mathcal{Y})
$$

maps the time-varying Hardy space $H^{2}\left(\left\{\mathcal{U}_{k}\right\}_{k \geq 0}\right):=\bigoplus_{k=0}^{\infty} S_{1}^{k} \mathcal{U}_{k}$ (where $\mathbf{u}=\bigoplus_{k=0}^{\infty} z^{k} u_{k} \in H^{2}\left(\left\{\mathcal{U}_{k}\right\}\right)$ is assigned the Hardy-space norm $\|\mathbf{u}\|^{2}=$ $\left.\sum_{k=0}^{\infty}\left\|u_{k}\right\|^{2}\right)$ isometrically into $H_{\boldsymbol{\beta}}^{2}(\mathcal{Y})$, and

$\left(2^{\prime}\right)$ there is a strictly lower triangular matrix $L=\left[L_{i j}\right]_{i, j=0,1,2, \ldots}$ (so $L_{i j}=0$ for $\left.i \leq j\right)$ with entries $L_{i j} \in \mathcal{L}\left(\mathcal{U}_{j}, \mathcal{U}_{i}\right)$ so that

$$
S_{\boldsymbol{\beta}}\left[\begin{array}{lllll}
M_{\Theta_{0}} & M_{\Theta_{1}} S_{1} & M_{\Theta_{2}} S_{1}^{2} & \cdots
\end{array}\right]=\left[\begin{array}{llll}
M_{\Theta_{0}} & M_{\Theta_{1}} S_{1} & M_{\Theta_{2}} S_{1}^{2} & \cdots
\end{array}\right] L .
$$

We conclude that if $\left\{\Theta_{k}\right\}_{k \geq 0}$ is a $\boldsymbol{\beta}$-inner function family and if we set

$$
\mathcal{M}=\bigoplus_{k=0}^{\infty} \Theta_{k} S_{1}^{k} \mathcal{U}_{k} \subset H_{\boldsymbol{\beta}}^{2}(\mathcal{Y})
$$

it then follows that $\mathcal{M}$ is $S_{\boldsymbol{\beta}}$-invariant and that the multiplication operator

$$
M_{\Theta}=\left[\begin{array}{llll}
M_{\Theta_{0}} & M_{\Theta_{1}} & M_{\Theta_{2}} & \cdots
\end{array}\right]: \bigoplus_{k=0}^{\infty} S_{1}^{k} \mathcal{U}_{k} \rightarrow H_{\boldsymbol{\beta}}^{2}(\mathcal{Y})
$$


maps the time-varying Hardy space $H^{2}\left(\left\{\mathcal{U}_{k}\right\}_{k \geq 0}\right):=\bigoplus_{k=0}^{\infty} S_{1}^{k} \mathcal{U}_{k}$ unitarily onto the $S_{\boldsymbol{\beta}}$-invariant subspace $\mathcal{M} \subset H_{\boldsymbol{\beta}}^{2}(\mathcal{Y})$. Putting all the pieces together, we arrive at the following converse of all these observations which amounts to our second analogue of the Beurling-Lax theorem for the weighted Hardy space setting. To follow the statement the reader should refer back to Definitions 2.1 and 5.7 .

Theorem 6.9. Let $\mathcal{M}$ be a closed $S_{\boldsymbol{\beta}}$-invariant subspace of $H_{\boldsymbol{\beta}}^{2}(\mathcal{Y})$. Then there is a $\boldsymbol{\beta}$-inner function family $\left\{\Theta_{k}\right\}_{k \geq 0}$ so that $\mathcal{M}=M_{\Theta} H^{2}\left(\left\{\mathcal{U}_{k}\right\}_{k \geq 0}\right)$ (with $M_{\Theta}$ as in (6.15)).

Furthermore, given the $S_{\boldsymbol{\beta}}$-invariant closed subspace $\mathcal{M} \subset H_{\boldsymbol{\beta}}^{2}(\mathcal{Y})$, a $\boldsymbol{\beta}$ unitary colligation family realization $\left\{U_{k}=\left[\begin{array}{cc}A & B_{k} \\ C & D_{k}\end{array}\right]\right\}_{k \geq 0}$ for the $\boldsymbol{\beta}$-inner function family representer $\left\{\Theta_{k}\right\}_{k \geq 0}$ for $\mathcal{M}$ can be constructed according to the following algorithm:

(1) Set $\mathcal{X}=\mathcal{M}^{\perp}$ and define $A \in \mathcal{L}(\mathcal{X})$ and $C \in \mathcal{L}(\mathcal{X}, \mathcal{Y})$ by

$$
A=\left.S_{\boldsymbol{\beta}}^{*}\right|_{\mathcal{M}^{\perp}}, \quad C f=f(0) \quad \text { for } \quad f \in \mathcal{M}^{\perp} .
$$

(2) Construct $\left[\begin{array}{l}B_{k} \\ D_{k}\end{array}\right]$ by solving the Cholesky factorization problem (5.20) in Lemma 5.5.

(3) Set $\Theta_{k}(z)=\beta_{k}^{-1} D_{k}+z C R_{\boldsymbol{\beta}, k+1}(z A) B_{k}$.

Then $\left\{\Theta_{k}\right\}_{k \geq 0}$ is a $\boldsymbol{\beta}$-inner function family and any $\boldsymbol{\beta}$-inner function family arises in this way.

Remark 6.10. If $\left\{U_{k}\right\}_{k \geq 0}$ is the $\boldsymbol{\beta}$-unitary colligation family constructed as in Theorem 6.9, then the associated input-output map $\mathbf{T}_{\boldsymbol{\beta}}(2.5)$ is isometric from $\bigoplus_{k=0}^{\infty} \mathcal{U}_{k}$ into $\ell_{\boldsymbol{\beta}}^{2}(\mathcal{Y})$; this fact follows directly from Corollary 5.8 and requires only the $\boldsymbol{\beta}$-isometric property of $\left\{U_{k}\right\}_{k \geq 0}$. From the fact that $\mathcal{M}=$ $M_{\Theta} H^{2}\left(\left\{\mathcal{U}_{k}\right\}_{k \geq 0}\right)$ is $S_{\boldsymbol{\beta}}$-invariant, it follows that the image of $\mathcal{M}$ under the inverse $Z$-transform

$$
\mathcal{M}^{\vee}=\left\{\{y(k)\}_{k \geq 0}: \sum_{k=0}^{\infty} y(k) z^{k} \in \mathcal{M}\right\}=\mathbf{T}_{\boldsymbol{\beta}}\left(\bigoplus_{k=0}^{\infty} \mathcal{U}_{k}\right) \subset \ell_{\boldsymbol{\beta}}^{2}(\mathcal{Y})
$$

is invariant under the inverse $Z$-transform version $S_{\boldsymbol{\beta}}^{\vee}$ of $S_{\boldsymbol{\beta}}$ acting on $\ell_{\boldsymbol{\beta}}^{2}(\mathcal{Y})$ :

$$
S_{\boldsymbol{\beta}}^{\vee}:\{y(0), y(1), y(2), \ldots) \mapsto(0, y(0), y(1), \ldots) .
$$

From the arguments above one can see that the $S_{\boldsymbol{\beta}}^{\vee}$-invariance property of $\mathcal{M}^{\vee}$ is a consequence of the fact that $\left\{U_{k}\right\}_{k \geq 0}$ satisfies the additional weighted coisometry property (5.17).

6.3. Wandering-subspace $\boldsymbol{\beta}$-inner functions and Beurling-Lax representations. If the subspace $\mathcal{M} \subset H_{\boldsymbol{\beta}}^{2}(\mathcal{Y})$ is $S_{\boldsymbol{\beta}}$-invariant, then the subspace $\mathcal{E}:=\mathcal{M} \ominus S_{\boldsymbol{\beta}} \mathcal{M}$ has the property that $\mathcal{E} \subset \mathcal{M}$ and $\mathcal{E} \perp S_{\boldsymbol{\beta}} \mathcal{M}$. If it 
is the case that $\mathcal{E}$ generates $\mathcal{M}$ in the sense that $\mathcal{M}=\bigvee_{k \geq 0} S_{\boldsymbol{\beta}}^{k} \mathcal{E}$, then one says that $\mathcal{M}$ has the wandering subspace property (with wandering subspace equal to $\mathcal{E})$.

Letting $k=0$ in formula (6.11) and recalling formulas (3.4), we conclude that the reproducing kernel for the subspace $\mathcal{E}$ equals

$$
\begin{aligned}
K_{\mathcal{E}}(z, \zeta)= & I_{\mathcal{Y}}-C R_{\boldsymbol{\beta}}(z A) \mathcal{G}_{\boldsymbol{\beta}, C, A}^{-1} R_{\boldsymbol{\beta}}(\zeta A)^{*} C^{*} \\
& +z \bar{\zeta} C R_{\boldsymbol{\beta}, 1}(z A)\left(\mathfrak{G}_{\boldsymbol{\beta}, C, A}^{(1)}\right)^{-1} R_{\boldsymbol{\beta}, 1}(\zeta A)^{*} C^{*}
\end{aligned}
$$

Following [72, 74] we say that the function $\Theta$ is a wandering-subspace $\boldsymbol{\beta}$-inner function whenever

(1) $M_{\Theta}: \mathcal{U} \rightarrow H_{\boldsymbol{\beta}}^{2}(\mathcal{Y})$ is isometric, and

(2) $M_{\Theta} \mathcal{U}$ is orthogonal to $S_{\boldsymbol{\beta}}^{\ell} M_{\Theta} \mathcal{U}$ for $\ell \geq 1$.

In case $M_{\Theta} \mathcal{U}=\mathcal{E}$ is the wandering subspace for the $S_{\boldsymbol{\beta}}$-invariant subspace $\mathcal{M}$, then we have the Beurling-Lax-type representation of $\mathcal{M}$ as the closure of $\Theta \cdot \mathcal{U}[z]$, where $\mathcal{U}[z]$ denotes the collection of polynomials with coefficients in the Hilbert space $\mathcal{U}$. Construction of a wandering-subspace Bergman-inner function (or Bergman-inner function for short) for the wandering subspace $\mathcal{E}$ of $\mathcal{M}$ amounts to focusing on the first element $\Theta_{0}$ in the $\boldsymbol{\beta}$-inner function family associated with $\mathcal{M}$. Specifying Lemma 6.7 for the case $k=0$ then leads to the following. The special case $\beta_{j}=\frac{j !(n-1) !}{(n+j-1) !}$ is treated in [18]; closely related results for this special case were obtained earlier by Olofsson (see [74], especially Theorems 4.2 and 6.1 there).

Theorem 6.11. Given a $\boldsymbol{\beta}$-strongly stable $\boldsymbol{\beta}$-output-pair $(C, A)$, there exist operators $B \in \mathcal{L}(\mathcal{U}, \mathcal{X})$ and $D \in \mathcal{L}(\mathcal{U}, \mathcal{Y})$ which solve the Cholesky factorization problem

$$
\left[\begin{array}{l}
B \\
D
\end{array}\right]\left[\begin{array}{ll}
B^{*} & D^{*}
\end{array}\right]=\left[\begin{array}{cc}
\left(\mathfrak{G}_{\boldsymbol{\beta}, C, A}^{(1)}\right)^{-1} & 0 \\
0 & I_{\mathcal{Y}}
\end{array}\right]-\left[\begin{array}{l}
A \\
C
\end{array}\right] \mathcal{G}_{\boldsymbol{\beta}, C, A}^{-1}\left[\begin{array}{ll}
A^{*} & C^{*}
\end{array}\right]
$$

Moreover, if $\Theta$ is defined by

$$
\Theta(z)=D+z C R_{\boldsymbol{\beta}, 1}(z A) B
$$

where $\left[\begin{array}{l}B \\ D\end{array}\right]$ solves (5.20) (with $\left.k=0\right)$, then:

(1) The factorization $K_{\mathcal{E}}(z, \zeta)=\Theta(z) \Theta(\zeta)^{*}$ holds and therefore, the multiplication operator $M_{\Theta}$ maps $\mathcal{U}$ onto $\mathcal{E}$ unitarily.

(2) The subspace $\mathcal{E}$ is orthogonal to $S_{\boldsymbol{\beta}}^{k} M_{\Theta} \mathcal{U}$ for every $k \geq 1$.

(3) $M_{\Theta}$ is a contractive multiplier from the Hardy space $H^{2}(\mathcal{U})$ into $H_{\boldsymbol{\beta}}^{2}(\mathcal{Y})$. Hence, if $\mathcal{M}$ has the wandering subspace property, we conclude that $\mathcal{M}$ has the Beurling-Lax-type representation

$$
\mathcal{M}=H_{\boldsymbol{\beta}}^{2}(\mathcal{Y}) \text {-closure of } M_{\Theta} H^{2}(\mathcal{U}) \text {. }
$$


We note that the last statement in the theorem is a consequence of part (4) of Lemma 5.1 .

Remark 6.12. We note that our companion paper [18] also discusses several versions of Beurling-Lax-type representations for weighted Hardy spaces, but for the special case where $\boldsymbol{\beta}_{k}=\frac{k !(n-1) !}{(k+n-1) !}\left(\boldsymbol{\beta}=\boldsymbol{\beta}_{\alpha}\right.$ as in (1.6) with $\alpha=n$ a positive integer). In particular, Section 6.1 above is the generalization of the "second approach" from [18, and Section 6.2 above is the generalization of the "third approach" from [18]. The "first approach" from [18] uses the special form $\boldsymbol{\beta}=\boldsymbol{\beta}_{n}$ in a fundamental way and hence we have no analogue of the "first approach" for the general weight setting considered here.

\section{Weighted Hardy SPACES, CHARACTERISTiC FUnCtions, AND OPERATOR MODEL THEORY}

Theorem 6.9 explains how closed $S_{\boldsymbol{\beta}}$-invariant subspaces $\mathcal{M} \subset H_{\boldsymbol{\beta}}^{2}(\mathcal{Y})$ correspond to $\boldsymbol{\beta}$-inner function families $\left\{\Theta_{k}\right\}_{k \geq 0}$, including a transfer-functionlike realization $\Theta_{k}(z)=\boldsymbol{\beta}_{k}^{-1} D_{k}+z C R_{\boldsymbol{\beta}, k+1}(z A) B_{k}$ having a nice systemtheoretic interpretation. It will be convenient to introduce the following terminology.

Definition 7.1. We say that the Hilbert space operator $T \in \mathcal{L}(\mathcal{X})$ is a *- $\boldsymbol{\beta}$-hypercontraction if its adjoint $A=T^{*}$ is a $\boldsymbol{\beta}$-hypercontraction (see Definition (3.7). We say that $T$ is a $\boldsymbol{\beta}-C \cdot 0 *-\boldsymbol{\beta}$ hypercontraction if also $A=T^{*}$ is $\boldsymbol{\beta}$-strongly stable (see Definition 3.11).

From Lemma 4.1 it is easily seen that any operator $T$ of the form

$$
T=\left.P_{\mathcal{M}^{\perp}} S_{\boldsymbol{\beta}}\right|_{\mathcal{M}^{\perp}}
$$

for an $S_{\boldsymbol{\beta}}$-invariant subspace $\mathcal{M} \subset H_{\boldsymbol{\beta}}^{2}(\mathcal{Y})$ is a $\boldsymbol{\beta}$-C.0 $*$ - $\boldsymbol{\beta}$-hypercontraction. To complete our operator-model theory for the class of $\boldsymbol{\beta}$ - $C_{.0} *$ - $\boldsymbol{\beta}$-hypercontraction operators $T$, it remains to define a $\boldsymbol{\beta}$-inner characteristic function family $\left\{\Theta_{T, k}\right\}_{k \geq 0}$ for any $\boldsymbol{\beta}$ - $C_{.0} *$ - $\boldsymbol{\beta}$-hypercontraction operator $T$ with the property that we recover $T$ up to unitary equivalence via the formula (7.1) with $\mathcal{M}=M_{\Theta_{T}} H^{2}\left(\left\{\mathcal{U}_{k}\right\}_{k \geq 0}\right)$.

Let us suppose first only that $T \in \mathcal{L}(\mathcal{X})$ is a $*$ - $\boldsymbol{\beta}$-hypercontraction, i.e., $A:=T^{*}$ is a $\boldsymbol{\beta}$-hypercontraction. In particular, we then have that $\Gamma_{\boldsymbol{\beta}, A}\left[I_{\mathcal{X}}\right] \geq 0$ and hence $\Gamma_{\boldsymbol{\beta}, A}\left[I_{\mathcal{X}}\right]$ has a positive semidefinite square root, denoted as $D_{\boldsymbol{\beta}, A}:=\left(\Gamma_{\boldsymbol{\beta}, A}\left[I_{\mathcal{X}}\right]\right)^{1 / 2}$. Since $A$ is $\boldsymbol{\beta}$-hypercontractive, a consequence of the sufficiency side of part (1) of Theorem 3.6 (with $H=I_{\mathcal{X}}$ ) is that $\left(D_{\boldsymbol{\beta}, A}, A\right)$ is a $\boldsymbol{\beta}$-output stable pair which is also $\boldsymbol{\beta}$-isometric (i.e., the inequality (3.21) holds with equality) by construction. We then have all the shifted gramians $\mathfrak{G}_{\boldsymbol{\beta}, D_{\boldsymbol{\beta}, A}, A}^{(k)}$ given by (3.2) or (3.3) defined and positive semidefinite. Furthermore, by Proposition 3.1 we know that the weighted Stein identities (3.6) hold for $k=0,1,2, \ldots$. 
As in Remark 5.9, for $k=0,1,2, \ldots$ we let $\mathcal{X}_{k}$ be the completion of $\mathcal{X}$ in the $\mathcal{X}_{k}$-metric given by

$$
\left\langle x, x^{\prime}\right\rangle_{\mathcal{X}_{k}}:=\left\langle\mathfrak{G}_{\boldsymbol{\beta}, D_{\boldsymbol{\beta}, A}, A}^{(k)} x, x^{\prime}\right\rangle_{\mathcal{X}}
$$

where any elements of $\mathcal{X}$ having zero $\mathcal{X}_{k}$-norm are identified with zero. Similarly, we let $\mathcal{Y}_{k}$ denote the space $\mathcal{D}_{\boldsymbol{\beta}, A}:=\overline{\operatorname{Ran}} D_{\boldsymbol{\beta}, A}$ but with inner product given by

$$
\left\langle D_{\boldsymbol{\beta}, A} x, D_{\boldsymbol{\beta}, A} x^{\prime}\right\rangle_{\mathcal{Y}_{k}}=\beta_{k}^{-1} \cdot\left\langle D_{\boldsymbol{\beta}, A} x, D_{\boldsymbol{\beta}, A} x^{\prime}\right\rangle_{\mathcal{X}}
$$

We next let $\mathbf{A}_{k} \in \mathcal{L}\left(\mathcal{X}_{k}, \mathcal{X}_{k+1}\right)$ denote the operator $A \in \mathcal{L}(\mathcal{X})$, but viewed as acting from $\mathcal{X}_{k}$ into $\mathcal{X}_{k+1}$. Similarly, we let $\mathbf{C}_{k}$ denote the operator $D_{\boldsymbol{\beta}, A}$ but viewed as an operator acting from $\mathcal{X}_{k}$ into $\mathcal{Y}_{k}$. Due to the validity of the weighted Stein identities (3.6), we see that the operator $\left[\begin{array}{l}\mathbf{A}_{k} \\ \mathbf{C}_{k}\end{array}\right]$ extends uniquely to a well-defined isometry acting from $\mathcal{X}_{k}$ into $\left[\begin{array}{c}\mathcal{X}_{k+1} \\ \mathcal{Y}_{k}\end{array}\right]$. In particular, we see that there is a uniquely determined unitary transformation $\boldsymbol{\omega}_{k}: \mathcal{D}_{\mathbf{A}_{k}} \rightarrow \mathcal{Y}_{k}$ (here $\mathcal{D}_{\mathbf{A}_{k}}=\overline{\operatorname{Ran}} D_{\mathbf{A}_{k}}$ where we use the notation $D_{\mathbf{A}_{k}}=\left(I-\mathbf{A}_{k}^{*} \mathbf{A}_{k}\right)^{1 / 2}$ ) so that $\mathbf{C}_{k}=\boldsymbol{\omega}_{k} D_{A_{k}}$ (note that for $k=0$ we have $\mathfrak{G}_{\boldsymbol{\beta}, D_{\boldsymbol{\beta}, A}, A}^{(0)}=I_{\mathcal{X}}$ and $\left.\boldsymbol{\omega}_{0}=I_{\mathcal{D}_{\boldsymbol{\beta}, A}}\right)$. We next introduce the other defect operator $D_{T}^{(k)}:=D_{\mathbf{A}_{k}^{*}}=\left(I-\mathbf{A}_{k} \mathbf{A}_{k}^{*}\right)^{1 / 2}$ and the coefficient space $\mathcal{U}_{k}=\overline{\operatorname{Ran}}\left(D_{\mathbf{A}_{k}^{*}}\right)^{1 / 2}$. We then define a unitary colligation matrix $\mathbf{U}_{T, k}$ by

$$
\mathbf{U}_{T, k}=\left[\begin{array}{cc}
\mathbf{A}_{k} & D_{\mathbf{A}_{k}^{*}} \\
\mathbf{C}_{k} & -\boldsymbol{\omega}_{k} \mathbf{A}_{k}^{*}
\end{array}\right]:\left[\begin{array}{c}
\mathcal{X}_{k} \\
\mathcal{U}_{k}
\end{array}\right] \rightarrow\left[\begin{array}{c}
\mathcal{X}_{k+1} \\
\mathcal{Y}_{k}
\end{array}\right]
$$

Finally, we define the characteristic function family for the $*$ - $\boldsymbol{\beta}$-hypercontraction $T=A^{*}$ to be the family of functions $\left\{\boldsymbol{\Theta}_{T, k}\right\}_{k=0,1,2, \ldots}$ where

$$
\boldsymbol{\Theta}_{T, k}(z)=\left.\left(-\beta_{k}^{-1} \boldsymbol{\omega}_{k} \mathbf{A}_{k}^{*}+z \mathbf{C}_{k} R_{\boldsymbol{\beta}, k+1}\left(z \mathbf{A}_{k}\right) D_{\mathbf{A}_{k}^{*}}\right)\right|_{\mathcal{U}_{k}}: \mathcal{U}_{k} \rightarrow \mathcal{Y}_{k}
$$

We now impose the additional hypothesis that $T$ is $\boldsymbol{\beta}-C_{0}$, i.e., $A=T^{*}$ is $\boldsymbol{\beta}$-strongly stable. Then Lemma 3.13 tells us that $\left(D_{\boldsymbol{\beta}, A}, A\right)$ is exactly $\boldsymbol{\beta}$ observable, the zero-shifted gramian satisfies $\mathfrak{G}_{\boldsymbol{\beta}, D_{\boldsymbol{\beta}, A}, A}^{(0)}=\mathcal{G}_{\boldsymbol{\beta}, D_{\boldsymbol{\beta}, A}, A}=I_{\mathcal{X}}$, and as a consequence of Proposition 4.5 we have that all the shifted gramians $\mathfrak{G}_{\boldsymbol{\beta}, D_{\boldsymbol{\beta}, A}, A}^{(k)}$ are strictly positive definite. Then $\mathcal{X}_{k}$ and $\mathcal{X}$ are the same as sets, i.e., the inclusion maps $i^{(k)}: \mathcal{X} \rightarrow \mathcal{X}_{k}$ are all invertible for $k=0,1,2, \ldots$ If we introduce the adjusted colligation

$$
\begin{aligned}
U_{T, k} & =\left[\begin{array}{cc}
\left(i^{(k+1)}\right)^{-1} & 0 \\
0 & \beta_{k}^{1 / 2} I_{\mathcal{Y}}
\end{array}\right] \mathbf{U}_{T, k}\left[\begin{array}{cc}
i^{(k)} & 0 \\
0 & I_{\mathcal{U}_{k}}
\end{array}\right] \\
& =\left[\begin{array}{cc}
\left(i^{(k+1)}\right)^{-1} \mathbf{A}_{k} i^{(k)} & \left(i^{(k+1)}\right)^{-1} \mathbf{B}_{k} \\
\beta_{k}^{1 / 2} \mathbf{C}_{k} & \beta_{k}^{1 / 2} \mathbf{D}_{k}
\end{array}\right],
\end{aligned}
$$


then $U_{T, k}$ has the form

$$
U_{T, k}=\left[\begin{array}{cc}
A & B_{k} \\
C & D_{k}
\end{array}\right]:\left[\begin{array}{c}
\mathcal{X} \\
\mathcal{U}_{k}
\end{array}\right] \rightarrow\left[\begin{array}{c}
\mathcal{X} \\
\mathcal{D}_{\boldsymbol{\beta}, C, A}
\end{array}\right]
$$

and the unitary property of $\mathbf{U}_{T, k}$ translates to $U_{T, k}$ satisfying the relations (5.6) as well as (5.17). We then define the adjusted characteristic function family to be $\left\{\Theta_{T, k}\right\}$ where we set

$$
\Theta_{T, k}(z)=\beta_{k}^{-1} D_{k}+z C R_{\boldsymbol{\beta}, k+1}(z A) B_{k}: \mathcal{U}_{k} \rightarrow \mathcal{D}_{\boldsymbol{\beta}, C, A}
$$

where $A, C, B_{k}, D_{k}$ are determined ultimately from the $\boldsymbol{\beta}$ - $C .0 *$ - $\boldsymbol{\beta}$-hypercontraction as above.

Alternatively, given the $\boldsymbol{\beta}$ - $C_{.0} *-\boldsymbol{\beta}$-hypercontraction $T$, the characteristic function family $\left\{\Theta_{T, k}\right\}_{k \geq 0}$ can be defined more directly as follows. Set

$$
A=T^{*} \quad \text { and } \quad C=D_{\beta}, A .
$$

Then $(C, A)$ is an exactly $\boldsymbol{\beta}$-observable, $\boldsymbol{\beta}$-output stable (also $\boldsymbol{\beta}$-isometric) output pair. Construct operators $B_{k} \in \mathcal{L}(\mathcal{U}, \mathcal{X})$ and $D_{k} \in \mathcal{L}(\mathcal{U}, \mathcal{Y})$ with $\left[\begin{array}{l}B_{k} \\ D_{k}\end{array}\right]$ injective by solving the Cholesky factorization problem (5.20) as in Lemma 5.5, and then set $\Theta_{T, k}(z)=\beta_{k}^{-1} D_{k}+z C R_{\boldsymbol{\beta}, k+1}(z A) B_{k}$. Then $\Theta_{T, k}$ is uniquely determined by $T$ up to a unitary change-of-basis transformation $\sigma_{k}$ on the input space $\mathcal{U}_{k}$ for $k=0,1,2, \ldots$ We shall also call any such choice $\left\{\Theta_{T, k}\right\}_{k \geq 0}$ a characteristic function family for the $\boldsymbol{\beta}-C_{0^{-*}-\boldsymbol{\beta} \text { - }}$ hypercontraction $T$.

We note that the first element $\Theta_{T, 0}$ in the characteristic function family in the first form given above using the defect operators $D_{\mathbf{A}_{0}^{*}}$ for the special case where $\beta_{j}=\frac{j !(n-1) !}{(j+n-1) !}$ amounts to the characteristic function for the $C_{0}$. $n$-hypercontraction $A=T^{*}$ introduced and studied by Olofsson [72, 74].

Theorem 7.2. Suppose that $T \in \mathcal{L}(\mathcal{X})$ is a $\boldsymbol{\beta}$-C.0 $*$ - $\boldsymbol{\beta}$-hypercontraction as above. Let $\left\{\Theta_{T, k}\right\}_{k \geq 0}$ be the adjusted characteristic function family for $T$ as given by (7.3) and (7.2). Then $\left\{\Theta_{T, k}\right\}_{k \geq 0}$ is an inner function family and $T$ is unitarily equivalent to the operator $\left.P_{\mathcal{M}^{\perp}} S_{\boldsymbol{\beta}}\right|_{\mathcal{M}^{\perp}}$, where

$$
\mathcal{M}=\left[\begin{array}{llll}
M_{\Theta_{T, 0}} & M_{\Theta_{T, 1}} & M_{\Theta_{T, 2}} & \cdots
\end{array}\right] H^{2}\left(\left\{\mathcal{U}_{k}\right\}_{k \geq 0}\right)
$$

is the $S_{\boldsymbol{\beta}}$-invariant subspace associated with the inner function family $\left\{\Theta_{T, k}\right\}$.

Furthermore, if $T^{\prime}$ is another $\boldsymbol{\beta}-C_{0_{0}} * \boldsymbol{\beta}$-hypercontraction on a Hilbert space $\mathcal{X}^{\prime}$ with characteristic function family $\left\{\Theta_{T^{\prime}, k}\right\}$, then $T$ and $T^{\prime}$ are unitarily equivalent if and only if the adjusted characteristic function families $\left\{\Theta_{T, k}\right\}_{k \geq 0}$ and $\left\{\Theta_{T^{\prime}, k}\right\}_{k \geq 0}$ coincide in the following sense: for each $k=$ $0,1, \ldots$ there are unitary operators $\sigma_{k}: \mathcal{U}_{k} \rightarrow \mathcal{U}_{k}^{\prime}$ and $\tau: \mathcal{D}_{\boldsymbol{\beta}, A} \rightarrow \mathcal{D}_{\boldsymbol{\beta}, A^{\prime}}$ so that

$$
\tau \Theta_{T, k}(z)=\Theta_{T^{\prime}, k}(z) \sigma_{k} \quad \text { for each } \quad z \in \mathbb{D} .
$$


Proof. Suppose that $T \in \mathcal{L}(\mathcal{X})$ is a $\boldsymbol{\beta}$ - $C_{.0} *$ - $\boldsymbol{\beta}$-hypercontraction and set $A=T^{*}$. As was remarked in the introductory remarks to this section, it follows that $(C, A):=\left(D_{\boldsymbol{\beta}}, A\right)$ is an exactly $\boldsymbol{\beta}$-observable and $\boldsymbol{\beta}$-isometric output pair with associated gramian $\mathcal{G}_{\boldsymbol{\beta}, C, A}=\mathfrak{G}_{\boldsymbol{\beta}, C, A}^{(0)}=I_{\mathcal{X}}$. Theorem 4.3 then tells us that $A$ is unitarily equivalent to $S_{\boldsymbol{\beta}}^{*} \mid \operatorname{Ran} \mathcal{O}_{\beta, C, A}$. Then the subspace

$$
\mathcal{M}:=\left(\operatorname{Ran} \mathcal{O}_{\boldsymbol{\beta}, C, A}\right)^{\perp} \subset H_{\boldsymbol{\beta}}^{2}\left(\mathcal{D}_{\boldsymbol{\beta}, A}\right)
$$

is $S_{\boldsymbol{\beta}}$-invariant, and hence by Theorem 6.9 has a representation as $\mathcal{M}=$ $M_{\Theta} H^{2}\left(\left\{\mathcal{U}_{k}\right\}_{k \geq 0}\right)$ for $\Theta=\left[\begin{array}{llll}\Theta_{0} & \Theta_{1} & \Theta_{2} & \cdots\end{array}\right]$ equal to the multiplier associated with an inner function family $\left\{\Theta_{k}\right\}_{k \geq 0}$. Furthermore, the formulas $(7.3)$ and (7.2) for $\Theta_{k}$ amount to one possible way to construct the associated $\boldsymbol{\beta}$-inner function family $\left\{\Theta_{k}\right\}_{k \geq 0}$ according to the prescriptions of Theorem 6.9. It remains now only to verify the uniqueness statement.

Suppose first that $T$ and $T^{\prime}$ are unitarily equivalent $\boldsymbol{\beta}$ - $C_{0_{0}} *$ - $\boldsymbol{\beta}$-hypercontractions on Hilbert spaces $\mathcal{X}$ and $\mathcal{X}^{\prime}$ respectively. Thus there is a unitary operator $\omega: \mathcal{X} \rightarrow \mathcal{X}^{\prime}$ so that $\omega T=T^{\prime} \omega$, and hence also $\omega A=A^{\prime} \omega$ where $A=T^{*}$ and $A^{\prime}=T^{*}$. We next set $\tau=\left.\omega\right|_{\mathcal{D}_{\boldsymbol{\beta}, A}}$. Then it is easily verified that $\tau$ is unitary from $\mathcal{D}_{\boldsymbol{\beta}, A}$ onto $\mathcal{D}_{\boldsymbol{\beta}, A^{\prime}}$. The fact that the Cholesky factorization problem (5.20) has a unique injective solution up to a unitary transformation $\sigma_{k}: \mathcal{U} \rightarrow \mathcal{U}_{k}^{\prime}$ implies that the colligation matrices $U_{T, k}$ and $U_{T^{\prime}, k}$ are related by

$$
\left[\begin{array}{cc}
\omega & 0 \\
0 & \tau
\end{array}\right] U_{T, k}=U_{T^{\prime}, k}\left[\begin{array}{cc}
\omega & 0 \\
0 & \sigma_{k}
\end{array}\right] .
$$

From this relation it follows that $\left\{\Theta_{T, k}\right\}$ and $\left\{\Theta_{T^{\prime}, k}\right\}$ coincide in the sense given in the statement of the theorem.

Conversely, suppose that $\left\{\Theta_{T, k}\right\}$ and $\left\{\Theta_{T^{\prime}, k}\right\}$ coincide via unitary operators $\tau: \mathcal{D}_{\boldsymbol{\beta}, A} \rightarrow \mathcal{D}_{\boldsymbol{\beta}, A^{\prime}}$ and $\sigma_{k}: \mathcal{U}_{k} \rightarrow \mathcal{U}_{k}^{\prime}$. Set

$$
\mathcal{M}:=M_{\Theta} H_{\boldsymbol{\beta}}^{2}\left(\left\{\mathcal{U}_{T, k}\right\}_{k \geq 0}\right), \quad \mathcal{M}^{\prime}:=M_{\Theta} H_{\boldsymbol{\beta}}^{2}\left(\left\{\mathcal{U}_{T^{\prime}, k}\right\}_{k \geq 0}\right) .
$$

We know that the operator $A=T^{*}$ is unitarily equivalent to $\left.S_{\boldsymbol{\beta}}^{*}\right|_{\mathcal{M}^{\perp}}$ while $A^{\prime}=T^{*}$ is unitarily equivalent to $\left.S_{\boldsymbol{\beta}}^{*}\right|_{\mathcal{M}^{\prime \perp}}$. From Lemma 6.7 we see that $K_{S_{n}^{k} \mathcal{M} \ominus S_{\boldsymbol{\beta}}^{k+1}}(z, \zeta)=z^{k} \bar{\zeta}^{k} \Theta_{k}(z) \Theta_{k}(\zeta)^{*}$. Combining this with the decomposition (6.8) then gives

$$
K_{\mathcal{M}}(z, \zeta)=\sum_{k=0}^{\infty} z^{k} \bar{\zeta}^{k} \Theta_{k}(z) \Theta_{k}(\zeta)^{*}
$$

from which we get

$$
K_{\mathcal{M}^{\perp}}(z, \bar{\zeta})=R_{\boldsymbol{\beta}}(z \bar{\zeta}) I_{\mathcal{D}_{\boldsymbol{\beta}, A}}-\sum_{k=0}^{\infty} z^{k} \bar{\zeta}^{k} \Theta_{T, k}(z) \Theta_{T, k}(\zeta)^{*}
$$


A similar analysis gives the reproducing kernel for the subspace $\mathcal{M}^{\prime \perp}$ :

$$
K_{\mathcal{M}^{\prime \perp}}(z, \bar{\zeta})=R_{\boldsymbol{\beta}}(z \bar{\zeta}) I_{\mathcal{D}_{\boldsymbol{\beta}, A^{\prime}}}-\sum_{k=0}^{\infty} z^{k} \bar{\zeta}^{k} \Theta_{T^{\prime}, k}(z) \Theta_{T^{\prime}, k}(\zeta)^{*}
$$

The fact that $\left\{\Theta_{T, k}\right\}$ and $\left\{\Theta_{T^{\prime}, k}\right\}$ coincide then tells us that

$$
\tau K_{\mathcal{M}^{\perp}}(z, \bar{\zeta})=K_{\mathcal{M}^{\prime \perp}}(z, \bar{\zeta}) \tau .
$$

It is then easily seen that the map

$$
X: f(z) \mapsto \tau f(z)
$$

is unitary from $\mathcal{M}^{\perp}$ onto $\mathcal{M}^{\perp}$ and satisfies the intertwining relation

$$
X\left(\left.S_{\boldsymbol{\beta}}\right|_{\mathcal{M}^{\perp}}\right)^{*}=\left(\left.S_{\boldsymbol{\beta}}\right|_{\mathcal{M}^{\prime \perp}}\right)^{*} X
$$

and hence $\left(\left.S_{\boldsymbol{\beta}}\right|_{\mathcal{M}^{\perp}}\right)^{*}$ and $\left(\left.S_{\boldsymbol{\beta}}\right|_{\mathcal{M}^{\prime \perp}}\right)^{*}$ are unitarily equivalent. As it has already been observed that $A$ is unitarily equivalent to $\left(\left.S_{\boldsymbol{\beta}}\right|_{\mathcal{M}^{\perp}}\right)^{*}$ and $A^{\prime}$ is unitarily equivalent to $\left(\left.S_{\boldsymbol{\beta}}\right|_{\mathcal{M}^{\prime \perp}}\right)^{*}$, it follows that $A$ and $A^{\prime}$ (and hence also $T$ and $\left.T^{\prime}\right)$ are unitarily equivalent to each other.

Given a $\boldsymbol{\beta}$-inner function family $\left\{\Theta_{k}\right\}_{k \geq 0}$, then $\mathcal{M}:=M_{\Theta} H_{\boldsymbol{\beta}}^{2}\left(\left\{\mathcal{U}_{k}\right\}_{k \geq 0}\right)$ is a closed $S_{\boldsymbol{\beta}}$-invariant subspace of $H_{\boldsymbol{\beta}}^{2}(\mathcal{Y})$. We may then construct colligation matrices $U_{k}=\left[\begin{array}{ll}A & B_{k} \\ C & D_{k}\end{array}\right]$ satisfying the metric constraints (5.6) and (5.17) so that we also have $\mathcal{M}=M_{\Theta(\mathcal{M})} H^{2}\left(\left\{\mathcal{U}_{k}\right\}\right)$, where $\Theta(\mathcal{M})_{k}=\beta_{k}^{-1} D_{k}+$ $z C R_{\boldsymbol{\beta}, k+1}(z A) B_{k}$. A consequence of the uniqueness in Theorem 6.9 is that then $\Theta_{k}$ and $\Theta(\mathcal{M})_{k}$ are the same up to constant right unitary factor. Hence the realization for $\Theta(\mathcal{M})_{k}$ leads to a realization for $\Theta_{k}$. We next note that one can get a more direct route to this result and as a bonus get a canonical functional-model formulas for the operators $A, B_{k}, C, D_{k}$ for which $\Theta_{k}(z)=$ $\beta_{k}^{-1} D_{k}+z R_{\boldsymbol{\beta}, k+1}(z A) B_{k}$ as follows. We note that the specialization of this result to $k=0$ is closely related to Theorem 4.2 of Olofsson [74].

Theorem 7.3. Suppose that we are given an inner function family $\left\{\Theta_{k}\right\}_{k \geq 0}$ $\left(\right.$ say $\Theta_{k}(z): \mathcal{U}_{k} \rightarrow \mathcal{Y}$ for $\left.k=0,1, \ldots\right)$ generating the $S_{\boldsymbol{\beta}}$-invariant subspace

$$
\mathcal{M}=M_{\Theta} H^{2}\left(\left\{\mathcal{U}_{k}\right\}_{k \geq 0}\right) \subset H_{\boldsymbol{\beta}}^{2}(\mathcal{Y}) .
$$

Then a $\boldsymbol{\beta}$-unitary colligation family realization

$$
\left\{U_{k}=\left[\begin{array}{ll}
A & B_{k} \\
C & D_{k}
\end{array}\right]:\left[\begin{array}{c}
\mathcal{X} \\
\mathcal{U}_{k}
\end{array}\right] \rightarrow\left[\begin{array}{l}
\mathcal{X} \\
\mathcal{Y}
\end{array}\right]\right\}_{k \geq 0}
$$

for $\left\{\Theta_{k}\right\}_{k \geq 0}$ can be constructed as follows. Take $\mathcal{X}=\mathcal{M}^{\perp} \subset H_{\boldsymbol{\beta}}^{2}(\mathcal{Y})$ and

$$
U_{k}=\left[\begin{array}{cc}
\left.S_{\boldsymbol{\beta}}^{*}\right|_{\mathcal{M}^{\perp}} & \left.\left(S_{\boldsymbol{\beta}}^{k+1}\right)^{*} S_{\boldsymbol{\beta}}^{k} M_{\Theta_{k}}\right|_{\mathcal{U}_{k}} \\
\left.E\right|_{\mathcal{M}^{\perp}} & \beta_{k} \Theta_{k}(0)
\end{array}\right]:\left[\begin{array}{c}
\mathcal{M}^{\perp} \\
\mathcal{U}_{k}
\end{array}\right] \rightarrow\left[\begin{array}{c}
\mathcal{M}^{\perp} \\
\mathcal{Y}
\end{array}\right]
$$


Proof. Let us set

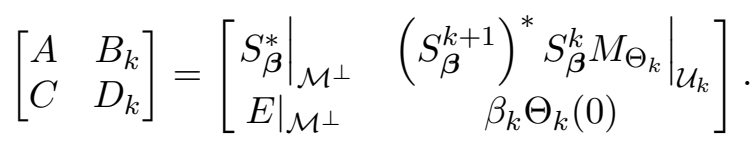

Before commencing the proof, let us point out some key identities. Let us write out the Taylor series for $\Theta_{k}$ as

$$
\Theta_{k}(z)=\sum_{j=0}^{\infty} \Theta_{k, j} z^{j}
$$

Then simple applications of formula (4.1) for the action of $S_{\boldsymbol{\beta}}^{* k}$ gives us the formulas

$$
\begin{aligned}
& \left(\left(S_{\boldsymbol{\beta}}^{k}\right)^{*} S_{\boldsymbol{\beta}}^{k} M_{\Theta_{k}} u\right)(z)=\sum_{j=0}^{\infty} \frac{\beta_{j+k}}{\beta_{j}}\left(\Theta_{k, j} u\right) z^{j}, \\
& \left(B_{k} u\right)(z)=\left(\left(S_{\boldsymbol{\beta}}^{k+1}\right)^{*} S_{\boldsymbol{\beta}}^{k} M_{\Theta_{k}} u\right)(z)=\sum_{j=0}^{\infty} \frac{\beta_{j+k+1}}{\beta_{j}}\left(\Theta_{k, j+1} u\right) z^{j} .
\end{aligned}
$$

As a first step toward the proof of Theorem 7.3, we verify that $B_{k}$ maps $\mathcal{U}_{k}$ into $\mathcal{M}^{\perp}$. Indeed, if $f \in \mathcal{M}$ and $u \in \mathcal{U}_{k}$, then

$$
\left\langle f,\left(S_{\boldsymbol{\beta}}^{k+1}\right)^{*} S_{\boldsymbol{\beta}}^{k} M_{\Theta_{k}} u\right\rangle_{H_{\boldsymbol{\beta}}^{2}(\mathcal{Y})}=\left\langle S_{\boldsymbol{\beta}}^{k+1} f, S_{\boldsymbol{\beta}}^{k} M_{\Theta_{k}} u\right\rangle_{H_{\boldsymbol{\beta}}^{2}(\mathcal{Y})}=0
$$

since $S_{\boldsymbol{\beta}}^{k} M_{\Theta_{k}} \mathcal{U}_{k} \perp S_{\boldsymbol{\beta}}^{k+1} \mathcal{M}$ by one of the defining properties of $\left\{\Theta_{k}\right\}_{k \geq 0}$ being an inner function family.

To verify the weighted isometry property (5.6) of $U_{k}$, it suffices to verify the three pieces

$$
\begin{aligned}
& A^{*} \mathfrak{G}_{\boldsymbol{\beta}, C, A}^{(k+1)} A+\beta_{k}^{-1} C^{*} C=\mathfrak{G}_{\boldsymbol{\beta}, C, A}^{(k)}, \\
& A^{*} \mathfrak{G}_{\boldsymbol{\beta}, C, A}^{(k+1)} B_{k}+\beta_{k}^{-1} C^{*} D_{k}=0, \\
& B_{k}^{*} \mathfrak{G}_{\boldsymbol{\beta}, C, A}^{(k+1)} B_{k}+\beta_{k}^{-1} D_{k}^{*} D_{k}=I_{\mathcal{U}_{k}} .
\end{aligned}
$$

To check (7.8), we first note the identity

$$
S_{\boldsymbol{\beta}} \mathfrak{G}_{\boldsymbol{\beta}, E, S_{\boldsymbol{\beta}}^{*}}^{(k+1)} S_{\boldsymbol{\beta}}^{*}+\beta_{k}^{-1} E^{*} E=\mathfrak{G}_{\boldsymbol{\beta}, E, S_{\boldsymbol{\beta}}^{*}}^{(k)}
$$

which is a consequence of Lemma 4.1 (2) and identity (3.6) in Proposition 3.1. Due to the $S_{\boldsymbol{\beta}^{*}}^{*}$-invariance of the subspace $\mathcal{M}^{\perp}$, we see that simple compression of the identity (7.11) to the subspace $\mathcal{M}^{\perp}$ gives us the identity (17.8). We next note that the identity (7.9) is equivalent to the validity of

$$
\left\langle\left[\begin{array}{c}
\mathfrak{G}_{\boldsymbol{\beta}, E, S_{\boldsymbol{\beta}}^{*}}^{(k+1)} S_{\boldsymbol{\beta}}^{*} f \\
f(0)
\end{array}\right],\left[\begin{array}{c}
B_{k} \\
\beta_{k}^{-1} D_{k}
\end{array}\right] u\right\rangle_{H_{\boldsymbol{\beta}}^{2}(\mathcal{Y}) \oplus \mathcal{Y}}=0
$$


for all $f \in \mathcal{M}^{\perp}$ and $u \in \mathcal{U}_{k}$. Let us rewrite the left-hand side of (17.12) as

$$
\begin{aligned}
\left\langle\mathfrak{G}_{\boldsymbol{\beta}, E, S_{\boldsymbol{\beta}}^{*}}^{(k+1)} S_{\boldsymbol{\beta}}^{*} f,\left(S_{\boldsymbol{\beta}}^{k+1}\right)^{*} S_{\boldsymbol{\beta}}^{k} M_{\Theta_{k}} u\right\rangle_{H_{\boldsymbol{\beta}}^{2}(\mathcal{Y})}+\left\langle E f, \Theta_{k}(0) u\right\rangle_{\mathcal{Y}} \\
=\left\langle S_{\boldsymbol{\beta}} \mathfrak{G}_{\boldsymbol{\beta}, E, S_{\boldsymbol{\beta}}^{*}}^{(k+1)} S_{\boldsymbol{\beta}}^{*} f,\left(S_{\boldsymbol{\beta}}^{k}\right)^{*} S_{\boldsymbol{\beta}}^{k} M_{\Theta_{k}} u\right\rangle_{H_{\boldsymbol{\beta}}^{2}(\mathcal{Y})}+\left\langle f(0), \Theta_{k}(0) u\right\rangle_{\mathcal{Y}} \\
=\left\langle\mathfrak{G}_{\boldsymbol{\beta}, E, S_{\boldsymbol{\beta}}^{*}}^{(k)} f,\left(S_{\boldsymbol{\beta}}^{k}\right)^{*} S_{\boldsymbol{\beta}}^{k} M_{\Theta_{k}} u\right\rangle_{H_{\boldsymbol{\beta}}^{2}(\mathcal{Y})}-\beta_{k}^{-1}\left\langle E f, E\left(S_{\boldsymbol{\beta}}^{k}\right)^{*} S_{\boldsymbol{\beta}}^{k} M_{\Theta_{k}} u\right\rangle_{\mathcal{Y}} \\
\quad+\left\langle f(0), \Theta_{k}(0) u\right\rangle_{\mathcal{Y}},
\end{aligned}
$$

where we used identity (7.11) for the second step. From (7.6) we see that

$$
E\left(S_{\beta}^{k}\right)^{*} S_{\boldsymbol{\beta}}^{k} M_{\Theta_{k}} u=\beta_{k} \Theta_{k, 0} u .
$$

Hence the last two terms in (7.13) cancel and it remains to show that the first term is zero, i.e., that

$$
\left\langle\mathfrak{G}_{\boldsymbol{\beta}, E, S_{\boldsymbol{\beta}}^{*}}^{(k)} f,\left(S_{\boldsymbol{\beta}}^{k}\right)^{*} S_{\boldsymbol{\beta}}^{k} M_{\Theta_{k}} u\right\rangle_{H_{\boldsymbol{\beta}}^{2}(\mathcal{Y})}=0 .
$$

Toward this end, we use the factorization (3.3) to see that

$$
\begin{aligned}
& \left\langle\mathfrak{G}_{\boldsymbol{\beta}, E, S_{\boldsymbol{\beta}}^{*}}^{(k)} f,\left(S_{\boldsymbol{\beta}}^{k}\right)^{*} S_{\boldsymbol{\beta}}^{k} M_{\Theta_{k}} u\right\rangle_{H_{\boldsymbol{\beta}}^{2}(\mathcal{Y})} \\
& =\left\langle S_{\boldsymbol{\beta}}^{k} \mathfrak{O}_{\boldsymbol{\beta}, E, S_{\boldsymbol{\beta}}^{*}}^{(k)} f, S_{\boldsymbol{\beta}}^{k} \mathfrak{O}_{\boldsymbol{\beta}, E, S_{\boldsymbol{\beta}}^{*}}^{(k)}\left(S_{\boldsymbol{\beta}}^{k}\right)^{*} S_{\boldsymbol{\beta}}^{k} M_{\Theta_{k}} u\right\rangle_{H_{\boldsymbol{\beta}}^{2}(\mathcal{Y})} .
\end{aligned}
$$

We now note that for $f \in \mathcal{M}^{\perp}$,

$$
S_{\boldsymbol{\beta}}^{k} \mathfrak{O}_{\boldsymbol{\beta}, E, S_{\boldsymbol{\beta}}^{*}}^{(k)} f=S_{\boldsymbol{\beta}}^{k} \mathfrak{D}_{\boldsymbol{\beta}, C, A}^{(k)} f .
$$

From (7.6) and (4.6) we see that

$$
\begin{aligned}
S_{\boldsymbol{\beta}}^{k} \mathfrak{D}_{\boldsymbol{\beta}, E, S_{\boldsymbol{\beta}}^{*}}^{(k)}\left(S_{\boldsymbol{\beta}}^{k}\right)^{*} S_{\boldsymbol{\beta}}^{k} M_{\Theta_{k}} u & =S_{\boldsymbol{\beta}}^{k} \mathfrak{D}_{\boldsymbol{\beta}, E, S_{\boldsymbol{\beta}}^{*}}^{(k)}\left(\sum_{j=0}^{\infty} \frac{\beta_{j+k}}{\beta_{j}} \Theta_{k, j} u z^{j}\right) \\
& =S_{\boldsymbol{\beta}}^{k} \sum_{j=0}^{\infty} \frac{\beta_{j}}{\beta_{j+k}} \cdot \frac{\beta_{j+k}}{\beta_{j}} \Theta_{k, j} u z^{j} \\
& =\sum_{j=0}^{\infty} \Theta_{k, j} u z^{j+k}=S_{\boldsymbol{\beta}}^{k} M_{\Theta_{k}} u .
\end{aligned}
$$

Combining (7.6), (7.16), and (7.17) gives us that

$$
\left\langle\mathfrak{G}_{\boldsymbol{\beta}, E, S_{\boldsymbol{\beta}}^{*}}^{(k)} f,\left(S_{\boldsymbol{\beta}}^{k}\right)^{*} S_{\boldsymbol{\beta}}^{k} M_{\Theta_{k}} u\right\rangle_{H_{\boldsymbol{\beta}}^{2}(\mathcal{Y})}=\left\langle S_{\boldsymbol{\beta}}^{k} \mathfrak{O}_{\boldsymbol{\beta}, C, A}^{(k)} f, S_{\boldsymbol{\beta}^{k}}^{k} M_{\Theta_{k}} u\right\rangle_{H_{\boldsymbol{\beta}}^{2}(\mathcal{Y})} .
$$

We now note that $S_{\boldsymbol{\beta}}^{k} \mathfrak{O}_{\boldsymbol{\beta}, C, A}^{(k)} f$ is orthogonal to $S_{\boldsymbol{\beta}}^{k} \mathcal{M}$ as a consequence of the decomposition (6.9). As $S_{\boldsymbol{\beta}}^{k} M_{\Theta_{k}} u \in S_{\boldsymbol{\beta}}^{k} \mathcal{M}$ by definition of $\mathcal{M}$ and the fact that $\Theta_{k}$ is an inner function family, we conclude that the right-hand side of (17.18) is zero, and (7.14) follows as needed. 
It remains to verify (7.10) which is equivalent to the validity of

$$
\left\langle\mathfrak{G}_{\boldsymbol{\beta}, C, A}^{(k+1)} B_{k} u, B_{k} u\right\rangle_{H_{\boldsymbol{\beta}}^{2}(\mathcal{Y})}+\beta_{k}^{-1}\left\|D_{k} u\right\|^{2}=\|u\|^{2}
$$

for all $u \in \mathcal{U}_{k}$. From (7.7) and (4.7) in the first term on the left-hand side of (7.19) we have

$$
\begin{aligned}
& \left\langle\mathfrak{G}_{\boldsymbol{\beta}, C, A}^{(k+1)} B_{k} u, B_{k} u\right\rangle_{H_{\boldsymbol{\beta}}^{2}(\mathcal{Y})} \\
& =\left\langle\mathfrak{G}_{\boldsymbol{\beta}, E, S_{\boldsymbol{\beta}}^{*}}^{(k+1)}\left(\sum_{j=0}^{\infty} \frac{\beta_{j+k+1}}{\beta_{j}} \Theta_{k, j+1} u z^{j}\right), \sum_{j=0}^{\infty} \frac{\beta_{j+k+1}}{\beta_{j}} \Theta_{k, j+1} u z^{j}\right\rangle_{H_{\boldsymbol{\beta}}^{2}(\mathcal{Y})} \\
& =\sum_{j=0}^{\infty} \frac{\beta_{j}^{2}}{\beta_{j+k+1}}\left(\frac{\beta_{j+k+1}}{\beta_{j}}\right)^{2}\left\langle\Theta_{k, j+1} u, \Theta_{k, j+1} u\right\rangle_{\mathcal{Y}} \\
& =\sum_{j=0}^{\infty} \beta_{j+k+1}\left\|\Theta_{k, j+1} u\right\|_{\mathcal{Y}}^{2}=\sum_{j=1}^{\infty} \beta_{j+k}\left\|\Theta_{k, j} u\right\|_{\mathcal{Y}}^{2} .
\end{aligned}
$$

On the other hand, from the definitions it is easily verified that

$$
\left\|S_{\boldsymbol{\beta}}^{k} M_{\Theta_{k}} u\right\|_{H_{\boldsymbol{\beta}}^{2}(\mathcal{Y})}^{2}=\sum_{j=0}^{\infty} \beta_{j+k}\left\|\Theta_{k, j} u\right\|_{\mathcal{Y}}^{2} .
$$

Combining this with the result of the preceding calculation gives

$$
\left\langle\mathfrak{G}_{\boldsymbol{\beta}, C, A}^{(k+1)} B_{k} u, B_{k} u\right\rangle_{H_{\boldsymbol{\beta}}^{2}(\mathcal{Y})}+\beta_{k}\left\|\Theta_{k, 0} u\right\|_{\mathcal{Y}}^{2}=\left\|S_{\boldsymbol{\beta}}^{k} M_{\Theta_{k}} u\right\|_{H_{\boldsymbol{\beta}}^{2}(\mathcal{Y})}^{2} .
$$

On the other hand we know that $\left\|S_{\beta}^{k} M_{\Theta_{k}} u\right\|_{H_{\boldsymbol{\beta}}^{2}(\mathcal{Y})}^{2}=\|u\|_{\mathcal{U}}^{2}$ by another one of the defining properties of $\left\{\Theta_{k}\right\}_{k \geq 0}$ being an inner function family. From the identity $D_{k}=\beta_{k} \Theta_{k}(0)=\beta_{k} \Theta_{k, 0}$, we see that $\beta_{k}^{-1}\left\|D_{k} u\right\|_{\mathcal{Y}}^{2}=\beta_{k}\left\|\Theta_{k, 0} u\right\|_{\mathcal{Y}}^{2}$. Combining all these observations now gives us (7.10) as wanted. This completes the proof of the weighted isometry property (5.6) for $U_{k}$.

To verify the weighted coisometry property (5.17), we note that, in view of the validity of the weighted isometry property (5.6) already checked, it suffices to show that the colligation matrix $U_{k}$ (7.4) maps $\mathcal{M}^{\perp} \oplus \mathcal{U}_{k}$ onto $\mathcal{M}^{\perp} \oplus \mathcal{Y}$. Toward this end we first consider the special case where $k=0$.

Let us therefore suppose that $\left[\begin{array}{l}g \\ y\end{array}\right]$ is an element of $\left[\begin{array}{c}\mathcal{M}^{\perp} \\ \mathcal{Y}\end{array}\right]$ which is orthogonal to $U_{0}\left[\begin{array}{l}\mathcal{M}^{\perp} \\ \mathcal{U}_{0}\end{array}\right]$ in the $\left[\begin{array}{cc}\mathfrak{G}_{\boldsymbol{\beta}, E, S_{\boldsymbol{\beta}}}^{(1)} & 0 \\ 0 & I_{\mathcal{Y}}\end{array}\right]$-metric on $\left[\begin{array}{c}\mathcal{M}^{\perp} \\ \mathcal{Y}\end{array}\right]$, i.e., we suppose that

$$
\left\langle\left[\begin{array}{cc}
\mathfrak{G}_{\boldsymbol{\beta}, E, S_{\boldsymbol{\beta}}}^{(1)} & 0 \\
0 & I_{\mathcal{Y}}
\end{array}\right]\left[\begin{array}{c}
S_{\boldsymbol{\beta}}^{*} f+S_{\boldsymbol{\beta}}^{*} M_{\Theta_{0}} u_{0} \\
f(0)+\Theta_{0}(0) u_{0}
\end{array}\right],\left[\begin{array}{l}
g \\
y
\end{array}\right]\right\rangle_{\mathcal{M}^{\perp} \oplus \mathcal{Y}}=0
$$


for all $f \in \mathcal{M}^{\perp}$ and $u_{0} \in \mathcal{U}_{0}$. Using the factorization (3.3), we may rewrite (7.20) in the form

$$
\left\langle S_{\boldsymbol{\beta}} \mathfrak{O}_{\boldsymbol{\beta}, E, S_{\boldsymbol{\beta}}^{*}}^{(1)} S_{\boldsymbol{\beta}}^{*}\left(f+M_{\Theta_{0}} u_{0}\right), S_{\boldsymbol{\beta}} \mathfrak{O}_{\boldsymbol{\beta}, E, S_{\boldsymbol{\beta}}^{*}}^{(1)} g\right\rangle+\left\langle f(0)+\Theta_{0}(0) u_{0}, y\right\rangle=0 .
$$

From the formulas (4.6) for $\mathfrak{D}_{\boldsymbol{\beta}, E, S_{\boldsymbol{\beta}}^{*}}^{(1)}$ and (4.1) for $S_{\boldsymbol{\beta}}^{*}$, it is easy to verify the identity

$$
\mathfrak{O}_{\boldsymbol{\beta}, E, S_{\boldsymbol{\beta}}^{*}}^{(1)} S_{\boldsymbol{\beta}}^{*}=\left(S_{\boldsymbol{\beta}}^{*} S_{\boldsymbol{\beta}}\right)^{-1} S_{\boldsymbol{\beta}}^{*}
$$

Note that the latter operator is well defined since $S_{\boldsymbol{\beta}}$ is left invertible as a consequence of the last condition in (1.7); in fact this latter operator is the Moore-Penrose left inverse for $S_{\boldsymbol{\beta}}$ with action the same as the Hardy-space adjoint $S_{1}^{*}$ of the Hardy-space shift operator $S_{1}$ :

$$
S_{1}^{*}: f(z)=\sum_{j=0}^{\infty} f_{j} z^{j} \mapsto \sum_{j=0}^{\infty} f_{j+1} z^{j} .
$$

For convenience we shall simply write $S_{1}^{*}$ for $\left(S_{\boldsymbol{\beta}}^{*} S_{\boldsymbol{\beta}}\right)^{-1} S_{\boldsymbol{\beta}}^{*}$ even when acting on elements of $H_{\boldsymbol{\beta}}^{2}(\mathcal{Y})$. We may therefore rewrite (7.21) as

$$
\left\langle S_{\boldsymbol{\beta}} S_{1}^{*}\left(f+M_{\Theta_{0}} u_{0}\right), S_{\boldsymbol{\beta}} \mathfrak{D}_{\boldsymbol{\beta}, E, S_{\boldsymbol{\beta}}^{*}}^{(1)} g\right\rangle_{H_{\boldsymbol{\beta}}^{2}(\mathcal{Y})}+\left\langle f(0)+\Theta_{0}(0) u_{0}, y\right\rangle_{\mathcal{Y}}=0 .
$$

Note next that

$$
f=f(0)+S_{\boldsymbol{\beta}} S_{1}^{*} f, \quad M_{\Theta_{0}} u_{0}=\Theta_{0}(0) u_{0}+S_{\boldsymbol{\beta}} S_{1}^{*} \Theta_{0} u_{0}
$$

and that constant functions are orthogonal to $\operatorname{Ran} S_{\boldsymbol{\beta}}$ in $H_{\boldsymbol{\beta}}^{2}(\mathcal{Y})$. We may therefore rewrite (7.22) as

$$
\left\langle f+\Theta_{0} u_{0}, y+S_{\boldsymbol{\beta}} \mathfrak{D}_{\boldsymbol{\beta}, E, S_{\boldsymbol{\beta}}^{*}}^{(1)} g\right\rangle_{H_{\boldsymbol{\beta}}^{2}(\mathcal{Y})}=0 .
$$

We now observe that the decomposition (6.7) (with $k=1$ ) and the fact that the inner family $\left\{\Theta_{k}\right\}_{k>0}$ generates $\mathcal{M}=M_{\Theta} H^{2}\left(\left\{\mathcal{U}_{k}\right\}_{k>0}\right)$ gives rise to the following two orthogonal decompositions for the space $\left(S_{\boldsymbol{\beta}} \mathcal{M}\right)^{\perp}$ :

$$
\begin{aligned}
& \left(S_{\boldsymbol{\beta}} \mathcal{M}\right)^{\perp}=\mathcal{M}^{\perp} \oplus M_{\Theta_{0}} \mathcal{U}_{0}
\end{aligned}
$$

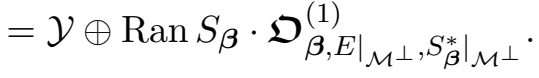

From the first decomposition in (7.24) we see that $f+\Theta_{0} u_{0}$ (with arbitrary $f \in \mathcal{M}^{\perp}$ and $\left.u_{0} \in \mathcal{U}_{0}\right)$ is a generic element of $\left(S_{\boldsymbol{\beta}} \mathcal{M}\right)^{\perp}$. From the second decomposition in (7.24) we see that $y+S_{\boldsymbol{\beta}} \mathfrak{D}_{\boldsymbol{\beta}, E, S_{\boldsymbol{\beta}}^{*}}^{(1)} g$ is an element of $\left(S_{\boldsymbol{\beta}} \mathcal{M}\right)^{\perp}$. The condition (7.23) holding for all $f \in \mathcal{M}^{\perp}$ and $u_{0} \in \mathcal{U}_{0}$ thus forces $y+S_{\boldsymbol{\beta}} \mathfrak{D}_{\boldsymbol{\beta}, E, S_{\boldsymbol{\beta}}^{*}}^{(1)} g=0$. As this decomposition is orthogonal in $H_{\boldsymbol{\beta}}^{2}(\mathcal{Y})$, we get $y=0$ and $S_{\boldsymbol{\beta}} \mathfrak{O}_{\boldsymbol{\beta} . E, S_{\boldsymbol{\beta}}^{*}}^{(1)} g=0$ individually. As $S_{\boldsymbol{\beta}}$ and $\mathfrak{O}_{\boldsymbol{\beta}, E, S_{\boldsymbol{\beta}}^{*}}^{(1)}$ are injective (see Proposition 4.5), we conclude that $g=0$ as well. This completes the verification that $U_{k}(\underline{7.4})$ is onto for the special case $k=0$. 
The next step is to show that the colligation matrix $U_{k}$ as in (7.4) is onto for a general $k>0$. To this end, we first view the shifted family $\left\{S_{\boldsymbol{\beta}}^{k} \Theta_{k+j}\right\}_{j \geq 0}$ as the inner family representing the shifted shift-invariant subspace $\widetilde{\mathcal{M}}^{(k)}:=$ $S_{\boldsymbol{\beta}}^{k} \mathcal{M}$ and apply the previous $k=0$ (now labeled as $j=0$ ) analysis to this adjusted setting. By the result of the previous paragraph we know that the adjusted 0-level colligation matrix

$$
\widetilde{U}_{0}^{(k)}:=\left[\begin{array}{cc}
A & B_{k} \\
C & D_{k}
\end{array}\right]=\left[\begin{array}{cc}
\left.S_{\boldsymbol{\beta}}^{*}\right|_{\widetilde{\mathcal{M}}^{(k) \perp}} & \left.S_{\boldsymbol{\beta}^{*}}^{*} S_{\boldsymbol{\beta}}^{k} M_{\Theta_{k}}\right|_{\mathcal{U}_{k}} \\
\left.E\right|_{\widetilde{\mathcal{M}}^{(k) \perp}} & 0
\end{array}\right]:\left[\begin{array}{c}
\widetilde{\mathcal{M}}^{(k) \perp} \\
\mathcal{U}_{k}
\end{array}\right] \rightarrow\left[\begin{array}{c}
\widetilde{\mathcal{M}}^{(k) \perp} \\
\mathcal{Y}
\end{array}\right]
$$

is onto. From the decomposition

$$
\mathcal{M}=\left(\oplus_{j=0}^{k-1} S_{\boldsymbol{\beta}}^{j} \mathcal{U}_{j}\right) \bigoplus S_{\boldsymbol{\beta}}^{k} \mathcal{M}
$$

we read off the following decomposition for $\widetilde{\mathcal{M}}^{(k) \perp}$ :

$$
\widetilde{\mathcal{M}}^{(k) \perp}=\mathcal{M}^{\perp} \bigoplus\left(\oplus_{j=0}^{k-1} S_{\boldsymbol{\beta}}^{j} \Theta_{j} \mathcal{U}_{j}\right) .
$$

Note that the operator $\left.S_{\boldsymbol{\beta}}^{j} M_{\Theta_{j}}\right|_{\mathcal{U}_{j}}$ is an isometric embedding of $\mathcal{U}_{j}$ onto $S_{\boldsymbol{\beta}}^{j} \Theta_{j} \mathcal{U}_{j} \subset H_{\boldsymbol{\beta}}^{2}(\mathcal{Y})$ for each $j$. This observation suggests that, in place of $\widetilde{U}^{(k)}$, we may analyze instead the operator $\widetilde{U}^{(k) \prime}$ given by

$$
\widetilde{U}^{(k) \prime}=\widetilde{U}^{(k)}\left[\begin{array}{lllll}
I_{\mathcal{M}^{\perp}} & & & & \\
& M_{\Theta_{0}} & & & \\
& & \ddots & & \\
& & & S_{\boldsymbol{\beta}}^{k-1} M_{\Theta_{k-1}} & S_{\boldsymbol{\beta}^{k}}^{k} M_{\Theta_{k}}
\end{array}\right]
$$

mapping $\mathcal{M}^{\perp} \oplus\left(\oplus_{j=0}^{k} \mathcal{U}_{j}\right)$ into $\mathcal{M}^{\perp} \oplus\left(\oplus_{j=0}^{k-1} S^{j} \Theta_{j} \mathcal{U}_{j}\right) \bigoplus \mathcal{Y}$. The fact noted above that $\widetilde{U}^{(k)}$ is onto tells us that $\widetilde{U}^{(k) \prime}$ is onto as well. Before proceeding further, we need to verify the following lemma.

Lemma 7.4. For $j$ any nonnegative integer,

$$
S_{\boldsymbol{\beta}}^{*} S_{\boldsymbol{\beta}}^{j+1} \Theta_{j+1} \mathcal{U}_{j+1} \subset \mathcal{M}^{\perp} \oplus S_{\boldsymbol{\beta}}^{j} \Theta_{j} \mathcal{U}_{j}
$$

Proof of Lemma 7.4. In view of the decomposition

$$
H_{\boldsymbol{\beta}}^{2}(\mathcal{Y})=\mathcal{M}^{\perp} \bigoplus\left(\oplus_{\ell=0}^{j} S_{\boldsymbol{\beta}}^{\ell} \Theta_{\ell} \mathcal{U}_{\ell}\right) \bigoplus S_{\boldsymbol{\beta}}^{j+1} \mathcal{M}
$$

it suffices to check, for any $u_{j+1} \in \mathcal{U}_{j+1}$, that

$$
\begin{aligned}
& \left\langle S_{\boldsymbol{\beta}}^{*} S_{\boldsymbol{\beta}}^{j+1} \Theta_{j+1} u_{j+1}, S_{\boldsymbol{\beta}}^{j+1} h\right\rangle=0 \text { for all } h \in \mathcal{M}, \\
& \left\langle S_{\boldsymbol{\beta}}^{*} S_{\boldsymbol{\beta}}^{j+1} \Theta_{j+1} u_{j+1}, S_{\boldsymbol{\beta}}^{\ell} \Theta_{\ell} u_{\ell}\right\rangle=0 \text { for all } 0 \leq \ell<j \text { and } u_{\ell} \in \mathcal{U}_{\ell} .
\end{aligned}
$$

As for (7.25), compute

$$
\left\langle S_{\boldsymbol{\beta}}^{*} S_{\boldsymbol{\beta}}^{j+1} \Theta_{j+1} u_{j+1}, S_{\boldsymbol{\beta}}^{j+1} h\right\rangle=\left\langle S_{\boldsymbol{\beta}}^{j+1} \Theta_{j+1} u_{j+1}, S_{\boldsymbol{\beta}}^{j+2} h\right\rangle=0,
$$


since $S_{\boldsymbol{\beta}}^{j+1} \Theta_{j+1} \mathcal{U}_{j+1} \perp S_{\boldsymbol{\beta}}^{j+2} \mathcal{M}$. As for (7.26)

$$
\left\langle S_{\boldsymbol{\beta}}^{*} S_{\boldsymbol{\beta}}^{j+1} \Theta_{j+1} u_{j+1}, S_{\boldsymbol{\beta}}^{\ell} \Theta_{\ell} u_{\ell}\right\rangle=0,
$$

since $S_{\boldsymbol{\beta}}^{\ell+1} \Theta_{\ell} u_{\ell} \perp S_{\boldsymbol{\beta}}^{j+1} \mathcal{M} \supset S_{\boldsymbol{\beta}}^{j+1} \Theta_{j+1} \mathcal{U}_{j+1}$ for $\ell<j$.

With the aid of the result of Lemma 7.4, we see that the colligation matrix $\widetilde{U}_{0}^{(k) \prime}$, when expanded out as a block matrix with respect to the decompositions $\mathcal{M}^{\perp} \bigoplus\left(\bigoplus_{j=0}^{k} \mathcal{U}_{j}\right)$ on the domain side and $\mathcal{M}^{\perp} \bigoplus\left(\bigoplus_{j=0}^{k-1} S_{\boldsymbol{\beta}}^{j} \Theta_{j} \mathcal{U}_{j}\right) \bigoplus \mathcal{Y}$ on the range side, is given by $\widetilde{U}^{(k) \prime}=$

$$
\left[\begin{array}{ccccc}
\left.S_{\boldsymbol{\beta}}^{*}\right|_{\mathcal{M}^{\perp}} & \left.S_{\boldsymbol{\beta}}^{*} M_{\Theta_{0}}\right|_{\mathcal{U}_{0}} & \left.P_{\mathcal{M}^{\perp}} S_{\boldsymbol{\beta}}^{*} S_{\boldsymbol{\beta}} M_{\Theta_{1}}\right|_{\mathcal{U}_{1}} & \cdots & \left.P_{\mathcal{M}^{\perp}} S_{\boldsymbol{\beta}}^{*} S_{\boldsymbol{\beta}}^{k} M_{\Theta_{k}}\right|_{\mathcal{U}_{k}} \\
& \left.P_{\Theta_{0} \mathcal{U}_{0}} S_{\boldsymbol{\beta}}^{*} S_{\boldsymbol{\beta}} M_{\Theta_{1}}\right|_{\mathcal{U}_{1}} & & \\
& & \ddots & \\
& & & \left.P_{S_{\boldsymbol{\beta}}^{k-1} \Theta_{k-1} \mathcal{U}_{k-1}} S_{\boldsymbol{\beta}}^{*} S_{\boldsymbol{\beta}^{k}}^{k} M_{\Theta_{k}}\right|_{\mathcal{U}_{k}} \\
\left.E\right|_{\mathcal{M}^{\perp}} & \left.E M_{\Theta_{0}}\right|_{\mathcal{U}_{0}} & & &
\end{array}\right]
$$

with all unspecified entries equal to 0 . As noted above, we know that $\widetilde{U}_{0}^{(k) \prime}$ is onto. This fact combined with the matrix structure of $\widetilde{U}_{0}^{(k) \prime}$ exhibited in (7.27) enables us to see that $P_{S_{\boldsymbol{\beta}}^{j-1} \Theta_{j-1} \mathcal{U}_{j-1}} S_{\boldsymbol{\beta}}^{*} S_{\boldsymbol{\beta}}^{j} M_{\Theta_{j}}$ maps $\mathcal{U}_{j}$ onto $S_{\boldsymbol{\beta}}^{j-1} M_{\Theta_{j-1}} \mathcal{U}_{j-1}$ for each $j=1,2, \ldots, k$. A simple induction argument then shows that $P_{\Theta_{0} \mathcal{U}_{0}} S_{\boldsymbol{\beta}}^{* k} S_{\boldsymbol{\beta}}^{k} M_{\Theta_{k}}$ maps $\mathcal{U}_{k}$ onto $\Theta_{0} \mathcal{U}_{0}$. We conclude that the block matrix

$$
\Xi^{(k)}:=\left[\begin{array}{cc}
I_{\mathcal{M}^{\perp}} & \left.P_{\mathcal{M}^{\perp}} S_{\boldsymbol{\beta}}^{* k} S_{\boldsymbol{\beta}}^{k} M_{\Theta_{k}}\right|_{\mathcal{U}_{k}} \\
0 & \left.P_{\Theta_{0} \mathcal{U}_{0}} S_{\boldsymbol{\beta}}^{* k} S_{\boldsymbol{\beta}^{k}}^{k} M_{\Theta_{k}}\right|_{\mathcal{U}_{k}}
\end{array}\right]:\left[\begin{array}{c}
\mathcal{M}^{\perp} \\
\mathcal{U}_{k}
\end{array}\right] \rightarrow\left[\begin{array}{c}
\mathcal{M}^{\perp} \\
\Theta_{0} \mathcal{U}_{0}
\end{array}\right]
$$

is onto. Let us use the identification map $M_{\Theta_{0}}: \mathcal{U}_{0} \rightarrow \Theta_{0} \mathcal{U}_{0}$ to introduce the adjusted 0-level colligation matrix

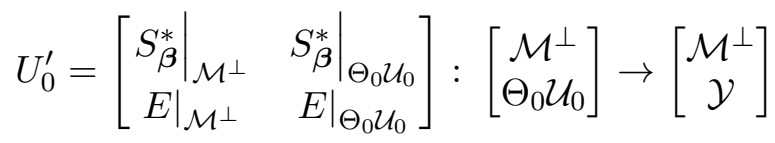

so that we have the factorization

$$
U_{0}=U_{0}^{\prime}\left[\begin{array}{cc}
I_{\mathcal{M}^{\perp}} & 0 \\
0 & \left.M_{\Theta_{0}}\right|_{\mathcal{U}_{0}}
\end{array}\right]
$$

(with $U_{0}$ as in (7.4) with $k=0$ ). By the $k=0$ case already handled, we know that $U_{0}$ is onto; it follows that $U_{0}^{\prime}$ is onto as well.

Next, we use the easily verified identity

$$
\left.E S_{\boldsymbol{\beta}}^{* k} S_{\boldsymbol{\beta}} M_{\Theta_{k}}\right|_{\mathcal{U}_{k}}=\beta_{k} \Theta_{k}(0)
$$


together with the definitions to verify the factorization formula for $U_{k}$ :

$$
U_{k}=U_{0}^{\prime} \Xi^{(k)}:\left[\begin{array}{c}
\mathcal{M}^{\perp} \\
\mathcal{U}_{k}
\end{array}\right] \rightarrow\left[\begin{array}{c}
\mathcal{M}^{\perp} \\
\mathcal{Y}
\end{array}\right]
$$

We now have exhibited $U_{k}$ as the composition of two onto linear operators and it finally follows that $U_{k}$ is also onto as wanted.

It remains now only to check that we recover $\Theta_{k}$ via the realization formula (2.10) or equivalently (5.9). We first note that, for $u \in \mathcal{U}_{k}$

$$
\begin{aligned}
C A^{j} B_{k} u & =E S_{\boldsymbol{\beta}}^{* j} B_{k} u(\text { by (7.5) }) \\
& \left.=E S_{\boldsymbol{\beta}}^{* j}\left(\sum_{\ell=0}^{\infty} \frac{\beta_{\ell+k+1}}{\beta_{\ell}} \Theta_{k, \ell+1} u z^{\ell}\right) \text { (using (17.7) }\right) \\
& \left.=E\left(\sum_{\ell=0}^{\infty} \frac{\beta_{\ell+j}}{\beta_{\ell}} \cdot \frac{\beta_{\ell+j+k+1}}{\beta_{\ell+j}} \Theta_{k, \ell+j+1} u z^{\ell}\right) \text { (using (4.1) }\right) \\
& =\beta_{j+k+1} \Theta_{k, j+1} u .
\end{aligned}
$$

Using this together with the formula $D_{k}=\beta_{k} \Theta_{k}(0)=\beta_{k} \Theta_{k, 0}$ then gives us, for $\lambda \in \mathbb{D}$,

$$
\begin{aligned}
\beta_{k}^{-1} D_{k} u+\sum_{j=0}^{\infty} \beta_{j+k+1}^{-1} C A^{j} B u \lambda^{j+1} & =\Theta_{k, 0} u+\sum_{j=0}^{\infty} \beta_{j+k+1}^{-1} \beta_{j+k+1} \Theta_{k, j+1} \lambda^{j+1} u \\
& =\Theta_{k}(\lambda) u
\end{aligned}
$$

and (5.9) follows as wanted.

\section{REFERENCES}

[1] M. B. Abrahamse and R. G. Douglas, A class of subnormal operators related to multiply connected domains, Advances in Math. 19 (1976), no. 1, 106-148.

[2] M. B. Abrahamse and R. G. Douglas, Operators on multiply connected domains, Proc. Roy. Irish Acad. Sect. A 74 (1974), 135-141.

[3] V. M. Adamjan, and D. Z. Arov, On unitary coupling of semiunitary operators, Dokl. Akad. Nauk. Arm. SSR XLIII 5 (1966), 257-263 [in Russian]. English Transl: Amer. Math. Soc. Transl. (2) 95 (1970), 75-129.

[4] J. Agler, The Arveson extension theorem and coanalytic models, Integral Equations Operator Theory 5 (1982), 608-631.

[5] J. Agler, Hypercontractions and subnormality, J. Operator Theory 13 (1985), no. 2, 203-217.

[6] J. Agler and J. E. McCarthy, Pick Interpolation and Hilbert Function Spaces, Graduate Studies in Mathematics 44, Amer. Math. Soc., Providence, 2002.

[7] A. Aleman, S. Richter and C. Sundberg, Beurling's theorem for the Bergman space, Acta Math. 177 (1996), no. 2, 275-310.

[8] D. Alpay, J. A. Ball, and Y. Peretz, System theory, operator models and scattering: the time-varying case, J. Operator Theory 47 (2002), 245-286.

[9] C.-G. Ambrozie, M. Engliš, and V. Müller, Operator tuples and analytic models over general domains in $\mathbb{C}^{n}$, J. Operator Theory 47 (2002), 287-302.

[10] J. Arazy and M. Engliš, Analytic models for commuting operator tuples on bounded symmetric domains, Trans. Amer. Math. Soc. 355 (2003) no. 2, 837-864. 
[11] N. Aronszajn, Theory of reproducing kernels, Trans. Amer. Math. Soc. 68 (1950), 337-404.

[12] W. B. Arveson, Subalgebras of $C^{*}$-Algebras, Acta Math. 123 (1969), 141-224.

[13] W. B. Arveson, Subalgebras of $C^{*}$-Algebras II, Acta Math.128 (1972), 271-308.

[14] W. B. Arveson, Subalgebras of $C^{*}$-algebras, III. Multivariable operator theory, Acta Math. 181 (1998), 159-228.

[15] A. Athavale, Model theory on the unit ball in $\mathbb{C}^{m}$, J. Operator Theory 27 (1992) no. 2, 347-358.

[16] J. A. Ball, Operators of class $C_{00}$ over multiply-connected domains, Michigan Math. J. 25 (1978) no. 2, 183-196.

[17] J. A. Ball and V. Bolotnikov, Interpolation problems for Schur multipliers on the Drury-Arveson space: from Nevanlinna-Pick to abstract interpolation problem, Integral Equations and Operator Theory 62 (2008), 301-349.

[18] J. A. Ball and V. Bolotnikov, Weighted Bergman spaces: shift-invariant subspaces and input/state/output linear systems, preprint arXiv:1209.3687.

[19] J. A. Ball and V. Bolotnikov, Canonical transfer-function realization for Schur multipliers on the Drury-Arveson space and models for commuting row contractions, Indiana Univ. Math. J., to appear.

[20] J. A. Ball, V. Bolotnikov, and Q. Fang, Multivariable backward-shift-invariant subspaces and observability operators, Multidimens. Syst. Signal Process. 18 (2007) no. 4, 191-248.

[21] J. A. Ball, V. Bolotnikov, and Q. Fang, Transfer-function realization for multipliers of the Arveson space, J. Math. Anal. Appl. 333 (2007) no. 1, 68-92.

[22] J.A. Ball and Q. Fang, Nevanlinna-Pick interpolation via graph spaces and Kreı̌nspace-space geometry, in: Mathematical Methods in Systems, Optimization, and Control (Ed. H. Dym, M.C. de Oliveira, and M. Putinar) pp. 43-71, OT 222, Birkhäuser, Basel, 2012.

[23] J. A. Ball and V. Vinnikov, Hardy spaces on a finite bordered Riemann surface, multivariable operator model theory and Fourier analysis along a unimodular curve, in: Systems, Approximation, Singular Integral Operators, and Related Topics (Ed. A. A. Borichev and N. K. Nikolskiॅ̌), pp. 37-56, OT 129 Birkhäuser 2001.

[24] J .A. Ball and V. Vinnikov, Overdetermined multidimensional systems: state space and frequency domain methods, in Mathematical Systems Theory in Biology, Communication, Computation, and Finance (Ed. J. Rosenthal and D. S. Gilliam) pp. 63-119, IMA Volumes in Mathematics and its Applications, Springer, 2003.

[25] J. A. Ball and V. Vinnikov, Formal reproducing kernel Hilbert spaces: the commutative and noncommutative settings, in Reproducing Kernel Spaces and Applications (Ed. D. Alpay), pp. 77-134, OT 143, Birkhäuser, Basel, 2003.

[26] J. A. Ball and V. Vinnikov, Lax-Phillips scattering and conservative linear systems: a Cuntz-algebra multidimensional setting, Mem. Amer. Math. Soc. Vol. 178 (2005) no. 837, Amer. Math. Soc., Providence, 2005.

[27] J. A. Ball and N. Cohen, de Branges-Rovnyak operator models and systems theory: a survey, in Topics in Matrix and Operator Theory, pp. 93-136, OT 50, BirkhäuserVerlag, 1991.

[28] A. Beurling, On two problems concerning linear transformations in Hilbert space, Acta Math. 81 (1949), 239-255.

[29] B. V. R. Bhat and T. Bhattacharyya, A model theory for q-commuting contractive tuples, J. Operator Theory 47 (2002), no. 1, 1551-1568.

[30] T. Bhattacharyya, J. Eschmeier and J. Sarkar, Characteristic function of a pure commuting contractive tuple, Integral Equations Operator Theory 53 (2005), no. 1, $23-32$.

[31] T. Bhattacharyya, J. Eschmeier and J. Sarkar, On c.n.c. commuting contractive tuples, Proc. Indian Acad. Sci. Math. Sci. 116 (2006), no. 3, 299-316. 
[32] L. de Branges and J. Rovnyak, Canonical models in quantum scattering theory, Perturbation Theory and its Applications in Quantum Mechanics (Ed. C. Wilcox), pp. 295-392, Wiley, New York, 1966.

[33] L. de Branges and J. Rovnyak, Square Summable Power Series, Holt, Rinehart and Winston, New York, 1966.

[34] M. S. Brodskii, Triangular and Jordan representations of linear operators, Transl. Math. Monographs 32, Amer. Math. Soc., Providence, 1971.

[35] J. W. Bunce, Models for n-tuples of noncommuting operators, J. Funct. Anal. 57 (1984), no. 1, 21-30.

[36] T. Constantinescu, Some aspects of nonstationarity I, Acta Sci. Math. (Szeged) 54 (1990) no. 3-4, 379-389.

[37] T. Constantinescu, Some aspects of nonstationarity II, Math. Balkanica (N.S.) 4 (1990) no. 3, 211-235.

[38] R. E. Curto and F.-H. Vasilescu, Standard operator models in the polydisc, Indiana Univ. Math. J. 42 (1990) no. 3, 791-810.

[39] R .E. Curto and F.-H. Vasilescu, Standard operator models in the polydisc, II, Indiana U. Math. J. 44 (1995) no. 3, 727-746.

[40] R. G. Douglas, Y.-S. Kim, H.-K. Kwon and J. Sarkar, Curvature invariant and generalized canonical operator models I, in: Spectral Theory, Mathematical System Theory, Evolution Equations, Differential and Difference Equations (Ed. W. Arendt, J.A. Ball, J. Behrndt, K.-H. Förster, and C. Trunk), pp. 293-304, OT 221 Birkhäuser, Basel, 2012.

[41] R. G. Douglas and V. I. Paulsen, Hilbert modules over function algebras, Pitman Research Notes in Mathematics Series 217, Longman Scientific \& Technical, Harlow, 1989.

[42] S. W. Drury, A generalization of von Neumann's inequality to the complex ball, Proc. Amer. Math. Soc. 68 (1978) no. 3, 300-304.

[43] P. Duren, D. Khavinson, H.S. Shapiro and C. Sundberg, Contractive zero-divisors in Bergman spaces, Pacific J. Math. 157 (1993), no. 1, 37-56.

[44] P. Duren and A. Schuster, Bergman Spaces, Mathematical Surveys and Monographs 100, Amer. Math. Soc., 2004.

[45] C. Foias, A. Frazho, I. Gohberg and M. A. Kaashoek, Metric Constrained Interpolation, Commutant Lifting and Systems, OT 100, Birkhäuser, Basel, 1998.

[46] A. E. Frazho, Models for noncommuting operators, J. Funct. Anal. 48 (1982), no.1, $1-11$.

[47] O. Giselsson and A. Olofsson, On some Bergman shift operators, Complex Anal. Oper. Theory 6 (2012), 829-842.

[48] P. R. Halmos, Shifts on Hilbert space, J. Reine Angew. Math. 208 (1961), 102-112.

[49] P. R. Halmos, A Hilbert Space Problem Book, Graduate Texts in Mathematics, 19, Springer-Verlag, New York-Berlin, 1982.

[50] H. Hedenmalm, A factorization theorem for square area-integrable analytic functions, J. Reine Angew. Math. 422 (1991), 45-68.

[51] H. Hedenmalm, A factoring theorem for the Bergman space, Bull. London Math. Soc. 26 (1994), no. 2, 113-126.

[52] H. Hedenmalm, S. Jakobsson and S. Shimorin, A biharmonic maximum principle for hyperbolic surfaces, J. Reine Angew. Math. 550 (2002), 2575.

[53] H. Hedenmalm, B. Korenblum, and K. Zhu, Theory of Bergman Spaces, Graduate Texts in Mathematics 199, Springer, 2000.

[54] J. W. Helton, The characteristic functions of operator theory and electrical network realization, Indiana Univ. Math. J. 22 (1972/1973), 403-414.

[55] J. W. Helton, Discrete time systems, operator models, and scattering theory, J. Funct. Anal. 16 (1974), 15-38.

[56] P.D. Lax, Translation invariant subspaces, Acta Math. 101 (1959), 163-178. 
[57] P. D. Lax and R. S. Phillips, Scattering Theory, Pure and Applied Math. 26, Academic Press, Boston, 1967.

[58] M. S. Livšic, On a class of linear operators in Hilbert space, Mat. Sbornik N.S. 19(61) (1946), 239-262; English transl.: Amer. Math. Soc. Transl. (2) 13 (1960), 61-83.

[59] M. S. Livšic, Operators, oscillations, waves (open systems), Transl. Math. Monographs 34, Amer. Math. Soc., Providence, 1973.

[60] M. S. Livšic, N. Kravitsky, A. S. Markus and V. Vinnikov, Theory of Commuting Nonselfadjoint Operators, Kluwer Academic Publishers, 1995.

[61] S. McCullough and S. Richter, Bergman-type reproducing kernels, contractive divisors, and dilations, J. Funct. Anal. 190 (2002), 447-480.

[62] S. McCullough and T. T. Trent, Invariant subspaces and Nevanlinna-Pick kernels, J. Funct. Anal. 178 (2000) no. 1, 226-249.

[63] P. S. Muhly, and B. Solel, Hilbert modules over operator algebras, Mem. Amer. Math. Soc. Vol. 117 (1995) no. 559, Amer. Math. Soc. , Providence, 1995.

[64] P. S. Muhly and B. Solel, Tensor algebras over $C^{*}$-correspondences: representations, dilations, and $C^{*}$-envelopes, J. Funct. Anal. 158 (1998) no. 2, 389-457.

[65] P. S. Muhly and B. Solel, Canonical models for representations of Hardy algebras, Integral Equations Operator Theory 53 (2005) no. 3, 411-452.

[66] V. Müller, Models for operators using weighted shifts, J. Operator Theory 20 (1988), no. $1,3-20$.

[67] V. Müller and F.-H. Vasilescu, Standard models for some commuting multioperators, Proc. Amer. Math. Soc. 117 (1991) no. 4, 979-989.

[68] B. Sz.-Nagy, C. Foias, H. Bercovici, and L. Kérchy, Harmonic Analysis of Operators on Hilbert Space, Second Edition, Springer, New York, 2010; first edition by B. Sz.Nagy and C. Foias, North-Holland and Akadémiai Kiadó, Amsterdam-Budapest, 1970.

[69] N. K. Nikolskii and V. I. Vasyunin, A unified approach to function models, and the transcription problem, in: The Gohberg Anniversary Collection Vol. 2 (Ed. H. Dym et al.), OT 41, Birkhäuser-Verlag, Basel-Boston, 1989, pp. 405-434.

[70] N. K. Nikolskii and V. I. Vasyunin, Elements of spectral theory in terms of the free function model Part I: Basic constructions, in: Holomorphic Spaces (Ed. S. Axler, J.E. McCarthy and D. Sarason), Mathematical Sciences Research Institute Publications Vol. 33, Cambridge University Press, 1998, pp. 211-302.

[71] A. Olofsson, Wandering subspace theorems, Integral Equations Operator Theory 51 (2005), no. 3, 395-409.

[72] A. Olofsson, A characteristic operator function for the class of $n$-hypercontractions, J. Funct. Anal. 236 (2006), 517-545.

[73] A. Olofsson, An operator-valued Berezin transform and the class of $n$ hypercontractions, Integral Equations Operator Theory 58 (2007), no. 4, 503-549.

[74] A. Olofsson Operator-valued Bergman inner functions as transfer functions, Algebra i Analiz 19 (2007), no. 4, 146-173.

[75] G. Popescu, Models for infinite sequences of noncommuting operators, Acta Sci. Math. 53 (1989), 355-368.

[76] G. Popescu, Characteristic functions for infinite sequences of noncommuting operators, J. Operator Theory 22 (1989), no.1, 51-71.

[77] G. Popescu, Isometric dilations for infinite sequences of noncommuting operators, Trans. Amer. Math. Soc. 316 (1989), 523-536.

[78] G. Popescu, Operator theory on noncommutative varieties, Indiana Univ. Math. J. 55 (2006) No. 2, 389-442.

[79] S. Pott, Standard models under polynomial positivity conditions, J. Operator Theory 41 (1999), no. 2, 365-389.

[80] A. Shields, Weighted shift operators and analytic function theory, in: Mathematical Surveys Volume 13 (Ed. C. Pearcy), pp. 49-128, Amer. Math. Soc., Providence, 1974. 
[81] S. Shimorin, Wold-type decompositions and wandering subspaces for operators close to isometries, J. Reine Angew. Math. 531 (2001), 147-189.

[82] S. Shimorin, On Beurling-type theorems in weighted $\ell^{2}$ and Bergman spaces, Proc. Amer. Math. Soc. 131 (2003), no. 6, 1777-1787.

[83] D.J. Sutton, Structure of Invariant Subspaces for Left-Invertible Operators on Hilbert Space, Virginia Tech PhD dissertation, August, 2010.

Department of Mathematics, Virginia Tech, Blacksburg, VA 24061-0123, USA

E-mail address: joball@math.vt.edu

Department of Mathematics, The College of William and Mary, WilliamsBURG VA 23187-8795, USA

E-mail address: vladi@math.wm.edu 